Aus dem Institut für Allgemeinmedizin

(Prof. Dr. med. E. Hummers)

der Medizinischen Fakultät der Universität Göttingen

\title{
Lässt sich die Anwendung von Schlaf- und Beruhigungsmitteln im Krankenhaus verringern?
}

Evaluation einer komplexen Intervention

\author{
INAUGURAL-DISSERTATION \\ zur Erlangung des Doktorgrades \\ der Medizinischen Fakultät der \\ Georg-August-Universität zu Göttingen
}

vorgelegt von

Jonas Peter Klemperer

aus

Bremen

Göttingen 2021 
Dekan:

\section{Betreuungsausschuss}

Betreuer:

Ko-Betreuer:

\section{Prüfungskommission}

Referent:

Ko-Referent:

Drittreferentin:

Datum der mündlichen Prüfung:
Prof. Dr. disc. pol. Wolfgang Himmel

Prof. Dr. med. W. Brück

Prof. Dr. disc. pol. Wolfgang Himmel

Prof. Dr. med. Peter Neumann

Prof. Dr. med. Peter Neumann

Prof. Dr. hum. biol. Margarete Schön 
Hiermit erkläre ich, die Dissertation mit dem Titel "Lässt sich die Anwendung von Schlaf- und Beruhigungsmitteln im Krankenhaus verringern? Evaluation einer komplexen Intervention" eigenständig angefertigt und keine anderen als die von mir angegebenen Quellen und Hilfsmittel verwendet zu haben.

Göttingen, den

(Unterschrift) 


\section{Inhaltsverzeichnis}

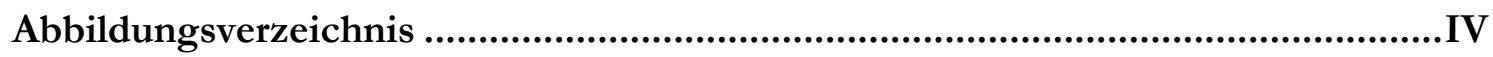

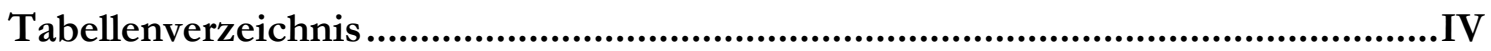

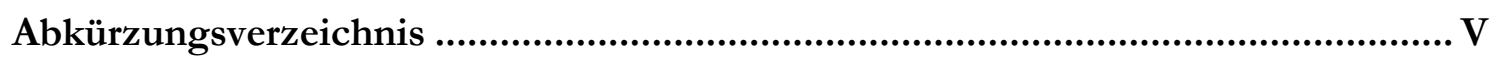

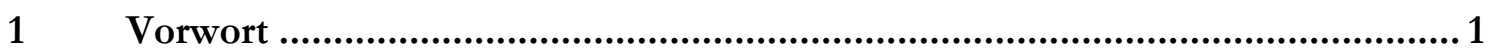

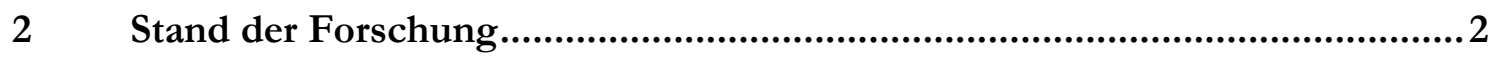

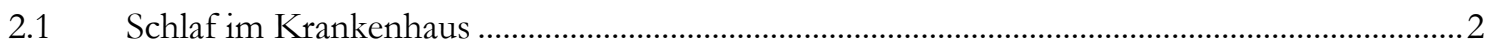

2.1.1 Pharmakologische Maßnahmen zur Förderung des Schlafs im Krankenhaus ...........................

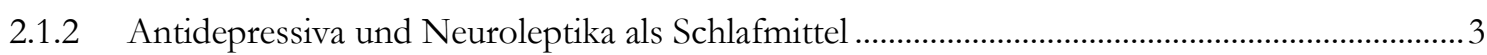

2.1.3 Nicht-pharmakologische Maßnahmen zur Förderung des Schlafs im Krankenhaus................ 5

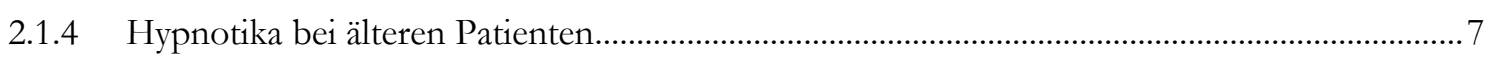

2.2 Interventionen zur Verbesserung potenziell inadäquater Medikation.......................................10

2.3 Screening-Tools für potenziell inadäquate Medikation................................................................12

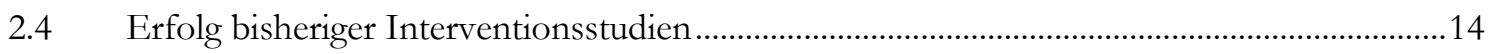

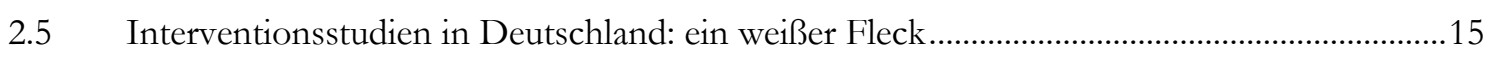

3 Forschungsfragen und Ziel der Studie ................................................. 16

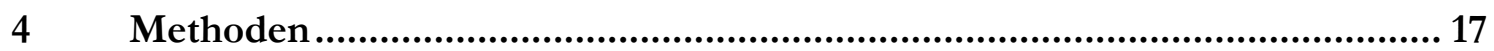

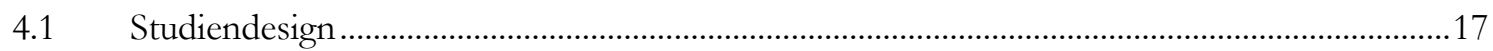

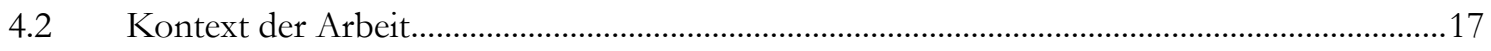

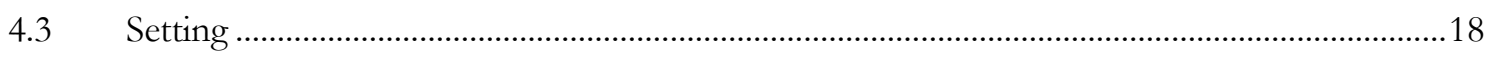

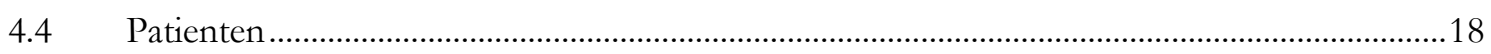

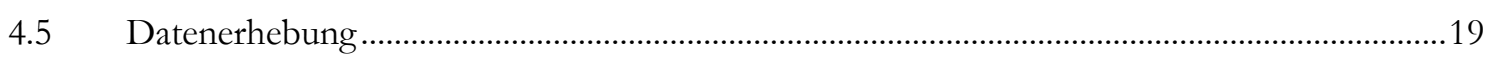

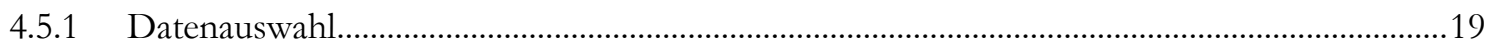

4.5.2 Klassifikation der Medikamente nach Wirkstoff..................................................................20

4.5.3 Bewertung nach potenziell inadäquater Medikation..................................................................2.

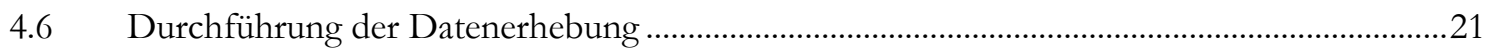

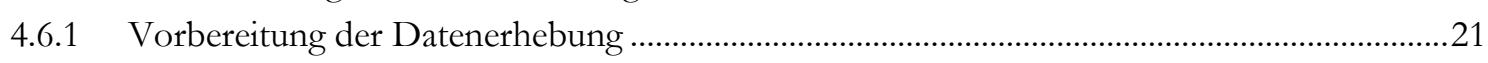

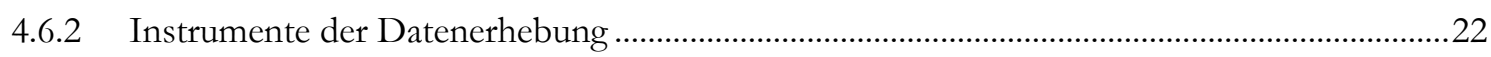

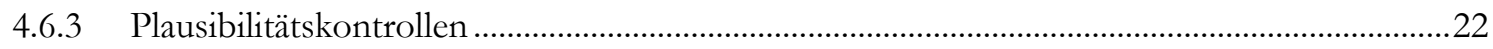

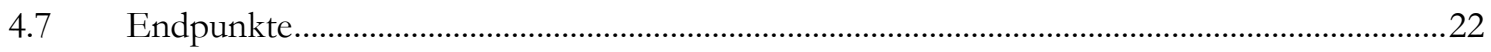

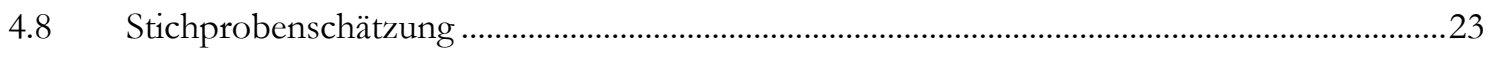

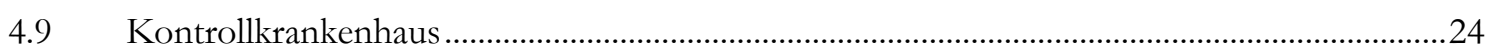

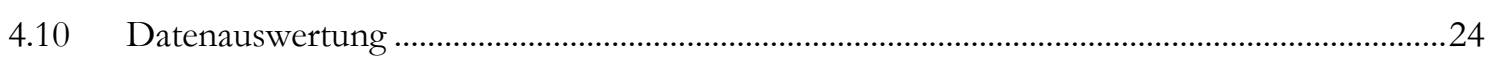

4.11 Genehmigung durch die Ethik-Kommission und Datenschutz...............................................25

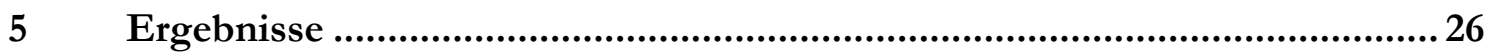




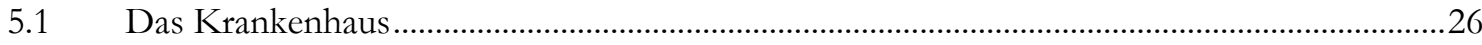

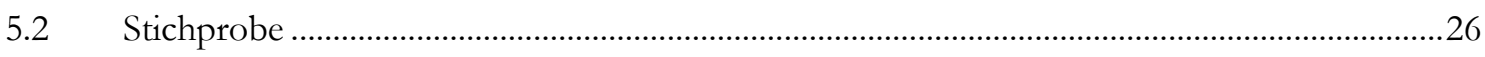

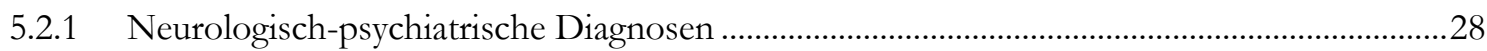

5.3 Menge und Angemessenheit der verabreichten schlafanstoßenden Medikamente ...............29

5.4 Schlafanstoßende Medikamente für Patienten mit und ohne neurologisch-

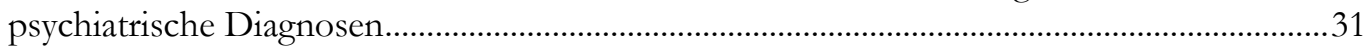

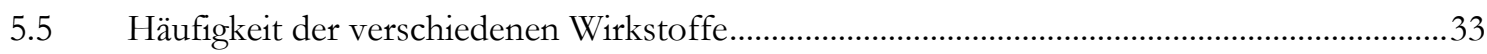

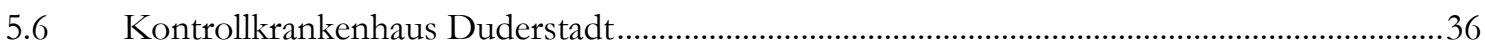

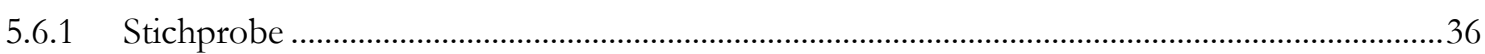

5.6.2 Menge der verabreichten schlafanstoßenden Medikamente im Kontrollkrankenhaus ..........38

$6 \quad$ Diskussion ........................................................................................ 40

S.1 Stärken und Schwächen der Untersuchung .........................................................................4 40

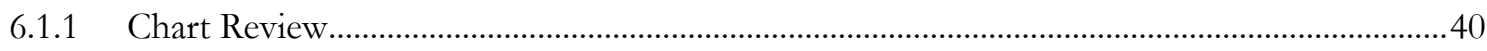

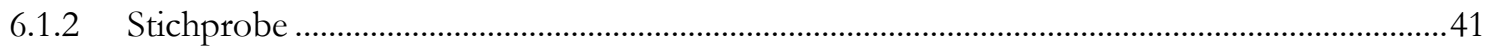

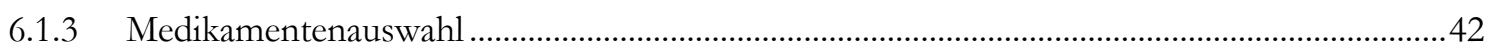

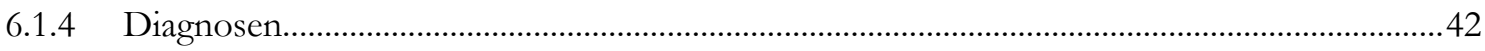

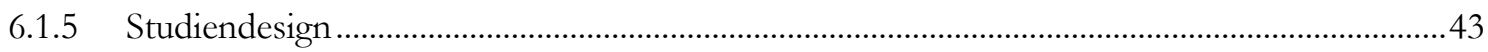

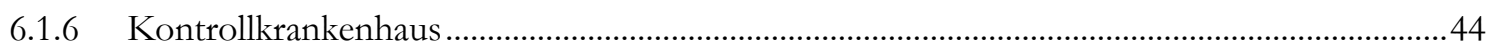

6.2 Menge der verabreichten schlafanstoßenden Medikamente ...................................................45

6.3 Angemessenheit der verabreichten schlafanstoßenden Medikamente ....................................46

6.4 Unterschiede in den Fachabteilungen .............................................................................. 47

6.5 Schlafanstoßende Medikamente für Patienten mit und ohne neurologischpsychiatrische Diagnosen...................................................................................................... 47

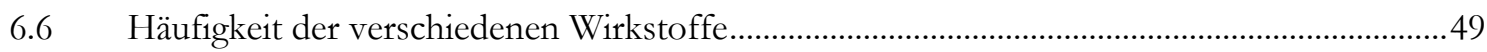

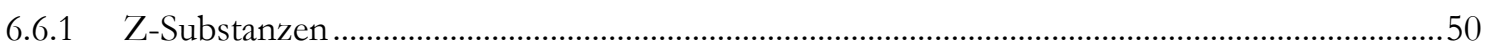

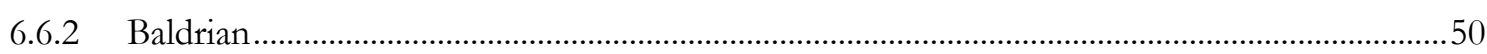

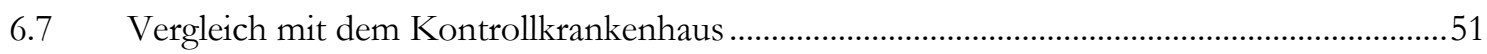

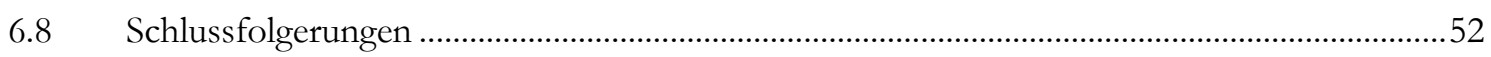

$7 \quad$ Zusammenfassung............................................................................. 53

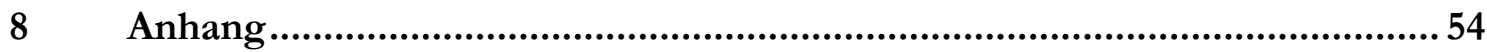

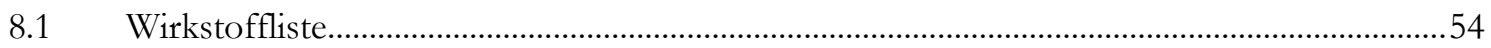

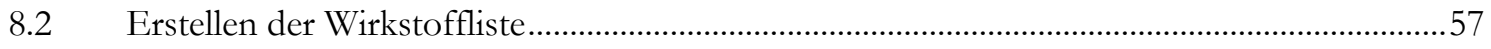

8.2.1 Definition der Hypnotika und Zusammenführen der Informationen ....................................57

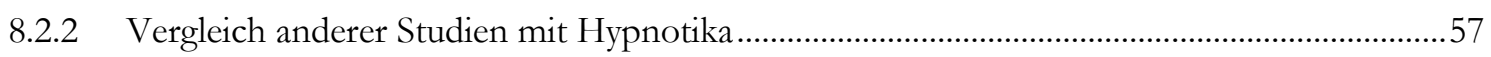

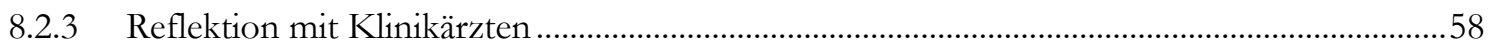

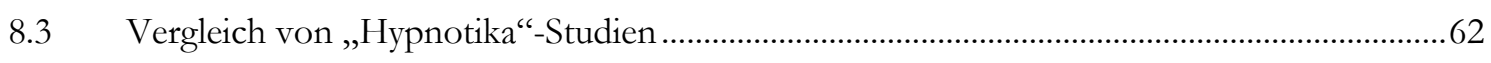

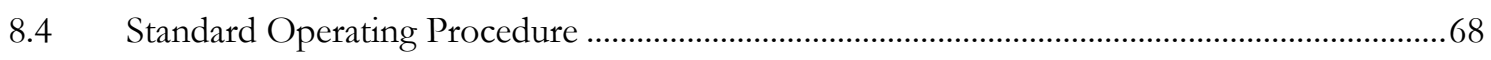

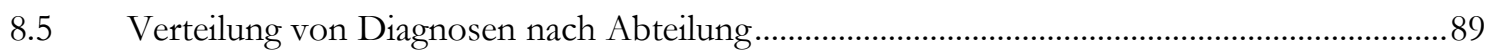

8.6 Ergebnisse mit Signifikanzwerten, Odds Ratios und Konfidenzintervallen..........................90

8.7 Vollständige Tabelle aller erhobenen Wirkstoffe .................................................................99

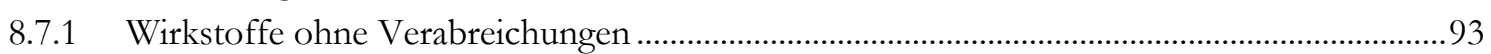


9 Literaturverzeichnis . 


\section{Abbildungsverzeichnis}

Abbildung 1 Patientenanteile mit schlafanstoßenden Medikamenten nach Wirkstoffgruppen.

\section{Tabellenverzeichnis}

Tabelle 1 „Die 10 Gebote für die Durchführung von Aktenauswertungsstudien“.....

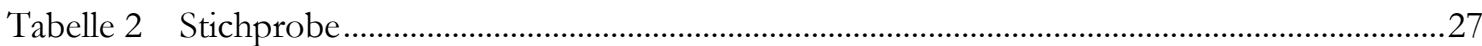

Tabelle 3 Neurologisch-psychiatrische Diagnosen..........................................................................28

Tabelle 4 Zielerreichung der primären und sekundären Endpunkte Prä- und Postintervention

Tabelle 5 Schlafanstoßende Medikamente; nach Stationen ..............................................................30

Tabelle 6 Schlafanstoßende Medikamente für Patienten mit und ohne neurologisch-

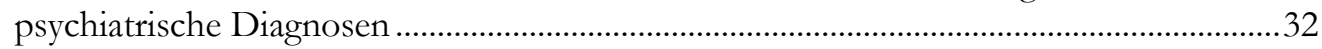

Tabelle 7 Tage mit Lormetazepam nach Abteilung und Zeitraum ..................................................34

Tabelle 8 Patientenanteile mit schlafanstoßenden Medikamenten nach Wirkstoffgruppen und potenziell inadäquater Dosierung .....................................................................................35

Tabelle 9 Stichprobe des Kontrollkrankenhauses ......................................................................37

Tabelle 10 Patienten mit neurologisch-psychiatrischen Diagnosen im Kontrollkrankenhaus ..........38

Tabelle 11 Patienten mit mindestens einem schlafanstoßenden Medikament im Kontrollkrankenhaus, mit und ohne neurologisch-psychiatrische Diagnosen 


\section{Abkürzungsverzeichnis}

PID

PIM

SOP
Potenziell inadäquate Dosierung

Potenziell inadäquate Medikation

Standard Operating Procedure 


\section{Vorwort}

Schlafprobleme, die Patienten ${ }^{*}$ während eines Krankenhausaufenthalts erfahren, werden oftmals mit schlafanstoßenden Medikamenten behandelt. Besonders bei älteren Patienten sollten diese allerdings sehr zurückhaltend verwendet werden - sonst kann es schnell zu unerwünschten Wirkungen kommen. Dennoch werden sie recht häufig verabreicht. Bereits vor 30 Jahren wurde auf Probleme beim Einsatz von Benzodiazepinen bei älteren Patienten hingewiesen. Während damals der aktuelle Forschungsstand oftmals nur schwer zu überschauen war, gibt es nun leicht zugängliche Informationen und Leitlinien für Ärzte und Patienten, in denen der aktuelle Wissensstand krankheits- oder symptombezogen aufgearbeitet ist. Um Verhaltensweisen zu ändern, ist aber mehr als der rein kognitive Input erforderlich, den die Leitlinien und Nachschlagewerke bieten.

Ein probates Mittel, um in der Gesundheitsforschung das Theoretische in die Praxis umzusetzen, sind Interventionsstudien. Mittlerweile gab es auch Interventionen mit dem Ziel, schlafanstoßende Medikamente für ältere Patienten zu reduzieren. Deren Erfolg wird aber oft nicht mit strengen Maßstäben gemessen. Bei der Beurteilung des Erfolgs einer Intervention sind zudem viele Stolperstricke zu beachten. So kann es nicht darum gehen, allen Patienten ein schlafanstoßendes Medikament zu verwehren. Die Intervention muss vielmehr an den richtigen Stellen - bei der Ziel-Patientengruppe - ankommen. Ein weiterer Stolperstrick bei der Betrachtung eines Rückgangs von Medikamenten kann sein, dass man eine Bewegung in die „richtige“ Richtung feststellt, dabei aber übersieht, dass eine solche Bewegung tatsächlich aber landes- oder sogar weltweit stattfindet.

Im Rahmen eines Projektes des Instituts für Allgemeinmedizin der Universitätsmedizin Göttingen und des Krankenhauses Göttingen-Weende wurden Beweggründe für den Einsatz schlafanstoßender Medikamente bei älteren Patienten auf ärztlicher, pflegerischer und Patienten-Seite untersucht. Basierend auf den Ergebnissen wurde eine auf mehreren Ebenen ansetzende Intervention entwickelt.

Ziel der vorliegenden Arbeit ist es, den Erfolg dieser Intervention anhand valider Daten zu messen. Gleichzeitig soll überprüft werden, ob ein möglicher Rückgang von schlafanstoßenden Medikamenten auch klinisch-pharmakologisch sinnvoll war, also die ,richtigen“ Patienten von der Intervention profitiert haben. Ebenso soll kontrolliert werden, ob ein möglicher Rückgang auch wirklich etwas mit der Intervention zu tun hatte und nicht einem allgemeinen Trend folgte, wie er auch in anderen Krankenhäusern stattfand.

\footnotetext{
* Im Folgenden wird - um den Lesefluss nicht zu unterbrechen - immer die männliche Form verwendet, die stets die weibliche einschließt, wenn nicht ausdrücklich anders vermerkt.
} 


\section{Stand der Forschung}

\subsection{Schlaf im Krankenhaus}

Mit fortschreitendem Alter verändert sich der Schlaf von Menschen. Insgesamt bekommen ältere Menschen weniger nächtlichen Schlaf. Typisch ist eine Abnahme der Schlafeffizienz, definiert als Verhältnis von Schlafenszeit zu der im Bett verbrachten Zeit. Die Abnahme der Schlafeffizienz kann durch vermehrtes nächtliches Erwachen erklärt werden. Neben den normalen Veränderungen im Alter können auch Krankheiten und Medikamente Ursachen für die Abnahme der Schlafeffizienz sein (Feinsilver und Hernandez 2017).

Ein Aufenthalt im Krankenhaus führt bei vielen Patienten zu schlechterem Schlaf. In einer kanadischen Studie schliefen Patienten im Krankenhaus durchschnittlich nur 5,5 Stunden, verglichen mit 7 Stunden zu Hause. Die Patienten berichten zudem von schlechter Schlafqualität im Krankenhaus (Dobing et al. 2016).

Auslöser für schlechteren Schlaf im Krankenhaus haben Young et al. (2008) ausführlich in einer Übersichtsarbeit dargestellt: Krankheiten, Medikamente und Krankenhausumgebung.

Beispiele für Krankheiten, die zu schlechterem Schlaf führen können, seien: chronisches Nierenversagen, Herzinsuffizienz, obstruktive Schlafapnoe sowie chronisch-obstruktiveLungenerkrankung. Ebenfalls können neurologische oder psychiatrische Erkrankungen den Schlaf beeinträchtigen wie auch Schmerz. Schlafbeeinträchtigende Medikamente seien Antiepileptika, selektive Serotonin-Inhibitoren, Monoaminoxidase-Hemmer, trizyklische Antidepressiva, Antihypertensiva, Antihistaminika und Kortikosteroide.

Die Krankenhausumgebung kann den Schlaf insbesondere durch laute Geräusche und das Wecken für Tätigkeiten der Krankenversorgung stören (Wesselius et al. 2018). Weiterhin sind Krankenhäuser prädestiniert für eine höhere Umgebungslautstärke durch eine zumeist gute Verkehrsanbindung, Notaufnahmen, die von Rettungswagen angefahren werden und Helikopterlandeplätzen. Auf den Stationen tragen Gespräche des Personals untereinander oder mit anderen Patienten zur höheren Umgebungslautstärke bei. In den Zimmern können Gespräche anderer Patienten, Smartphones, Fernseher und medizinische Technik Ursache für störende Geräusche sein.

Auf Intensivstationen, auf denen von vornherein ein höherer Lärmpegel herrscht, waren trotzdem Tätigkeiten der Patientenversorgung öfters der Grund für nächtliches Erwachen als die Umgebungslautstärke (Young et al. 2008, S. 479). Nächtliches Waschen und das Messen von Vitalparametern beispielsweise fanden im Durchschnitt 43 mal pro Nacht pro Patient statt (Tamburri et al. 2004). Auch nächtliche Toilettengänge - selbst oder von Mitpatienten - sind ein Faktor für nächtliches Erwachen im Krankenhaus (Wesselius et al. 2018). 
Die Entdeckung, dass Schlafmangel das Immunsystem beeinträchtigen kann, macht die Relevanz von ausreichendem Schlaf gerade auch für Krankenhauspatienten ersichtlich - insbesondere bei älteren Patienten, bei denen ein höheres Infektionsrisiko besteht (Bryant et al. 2004).

\subsubsection{Pharmakologische Maßnahmen zur Förderung des Schlafs im Krankenhaus}

Der Krankenhausaufenthalt - als Ausnahmesituation für den Patienten - führt potenziell zu Schlafproblemen und einer entsprechend höheren Nutzung von schlaffördernden Medikamenten.

Medikamente, die im Krankenhaus zur Verbesserung des Schlafs eingesetzt werden, stammen aus den Wirkstoffgruppen Benzodiazepine, Z-Substanzen, Antidepressiva, Neuroleptika und Phytotherapeutika (Steiger et al. 2019). Von den Antidepressiva und Neuroleptika werden jeweils Präparate mit sedierenden Eigenschaften genutzt (Riemann et al. 2017). In einer Befragung nannten Ärzte aus der Primärversorgung als Grund für die Verordnung von Benzodiazepinen das „geringere Übel“. Die Autoren schätzten die Situation so ein, dass die Ärzte von den psychosozialen Problemen der Patienten überwältigt sind und dadurch Empathie zeigen, dass sie Medikamente verschreiben. Sowohl Ärzte als auch Patienten ordnen Benzodiazepine als effektiv und vorteilhaft ein und sehen nur geringe Nebenwirkungs-Probleme (Anthierens et al. 2007). Schlafprobleme von Krankenhauspatienten unterscheiden sich noch einmal besonders von den ansonsten ,gesunder“"Menschen, da die Patienten aus dem häuslichen Kontext verlegt werden, zudem in einer akuten Krankheitssituation (Kanji et al. 2016).

Ob pharmakologische Interventionen (Hypnotika) den Schlaf von hospitalisierten Patienten tatsächlich verbessern, untersuchten Kanji et al. (2016) in einer systematischen Übersichtarbeit anhand von 15 Studien mit 861 Patienten. Sie prüften traditionelle Hypnotika, darunter Benzodiazepine und Z-Substanzen. Zu den betrachteten Endpunkten zählten Schlafeffizienz, Schlaflatenz (zum Einschlafen benötigte Zeit), Schlafunterbrechungen und Schlafphasen. Die Ergebnisse zeigen, dass die Schlaflatenz bei Patienten mit Schlafmitteln verkürzt war im Vergleich zu Patienten mit Placebo. Schlafeffizienz als auch Schlafunterbrechungen verbesserten sich aber nicht. Die Ergebnisse decken sich den Autoren zufolge mit der Expertenmeinung, dass Schlafmittel bei Einschlafstörungen hilfreich sein können, weniger allerdings bei Durchschlafstörungen. Schlafmittel seien also hilfreich, um schneller einzuschlafen - für eine Verbesserung anderer wichtiger Endpunkte der Schlafeffizienz und -qualität gäbe es keine Hinweise.

\subsubsection{Antidepressiva und Neuroleptika als Schlafmittel}

Der Einsatz von Antidepressiva und Neuroleptika, die traditionell nicht den Hypnotika zugeordnet werden, wurde in mehreren Studien beschrieben (Morlock et al. 2006; Wilson et al. 
2010; Everitt et al. 2014; Everitt et al. 2018). Vor- und Nachteile erläutern Enzensperger und Lehmann (2007):

Ein großer Vorteil der Antidepressiva sei demnach, dass sie - anders als Benzodiazepine keine muskelrelaxierende Wirkung haben, ein Auslöser für Stürze und Atemdepression. Der Einsatz von Neuroleptika sei von Vorteil bei psychiatrischen Grunderkrankungen mit Wahnvorstellungen, quälenden Gedanken und Ängsten. Sie hätten sich in der Behandlung von dementen, verwirrten, psychomotorisch erregten und agitierten Patienten bereits durchgesetzt. Sowohl Antidepressiva als auch Neuroleptika wird kein Suchtpotenzial nachgesagt, anders als Benzodiazepinen.

Nachteile bei der Behandlung mit Antidepressiva sind kardiovaskuläre Nebenwirkungen und die lange Wirkdauer sowie der verzögerte Wirkungseintritt. Je nach Wirkstoff können Neuroleptika anticholinerge (Mundtrockenheit, Steigerung der Körpertemperatur, Steigerung der Herzfrequenz, Harnblasenentleerungsstörungen) Wirkungen haben, die als störend wahrgenommen werden können, Blutbildveränderungen hervorrufen, kardiotoxisch sein oder extrapyramidal-motorische Nebenwirkungen (Störungen im Bewegungsablauf, die irreversibel sein können) verursachen.

Die Autoren beurteilen den Einsatz von Neuroleptika oder Antidepressiva als gerechtfertigt bei Patienten, die unter einer Schlafstörung leiden und gleichzeitig eine Depression haben, unter Unruhe- oder Erregungszuständen als Begleiterscheinung anderer Grunderkrankungen leiden, bei geriatrischen Patienten mit Verwirrtheit oder Demenz oder bei Patienten mit chronischem Schmerz.

Ob der Einsatz von Antidepressiva gegen Schlafstörungen hilft, wurde in einer CochraneÜbersichtsarbeit von Everitt et al. (2018) untersucht. Die Arbeit umfasst 23 RCTs mit 2806 Teilnehmern. Es wurden jeweils mit Placebo verglichen: Selektive Serotonin Wiederaufnahmehemmer, trizyklische Antidepressiva oder „andere“ Antidepressiva. Die Effekte von selektiven Serotonin-Wiederaufnahme-Hemmern auf den Schlaf seien unsicher und ließen kein klares Fazit zu, weil die vorhandenen Studien klein und die Nachbeobachtungszeiten kurz seien. Die Schlafqualität sei bei kurzzeitigem Gebrauch von Doxepin (trizyklisches Antidepressivum) und Trazodon (,anderes“ Antidepressivum) möglicherweise besser, verglichen mit Placebo. Für den langfristigen Einsatz von Antidepressiva gegen Schlafstörungen gäbe es keine Evidenz. Studien von höherer Qualität seien nötig.

Untersuchungen über den Einsatz von Neuroleptika wurden vor allem bei Patienten mit Schizophrenie durchgeführt (Riemann und Nissen 2012). Cohrs (2008) fasste den Forschungsstand über den Einfluss von Neuroleptika auf Schlafstörungen bei Patienten mit Schizophrenie zusammen. Die meisten Studien über Effekte der älteren Gruppe „typischer“ Neuroleptika sehen eine Steigerung der Gesamtschlafzeit und der Schlafeffizienz sowie unterschiedlich starke positive Auswirkungen auf tiefere erholsame Schlafstadien. Höhere Dosierungen typischer Neuroleptika über einen längeren Zeitraum scheinen eine Erhöhung der erholsamen tiefen REM-Schlafphase zu verursachen (Cohrs 2008, S. 951). Wirkstoffe aus 
der neueren Gruppe „atypischer" Neuroleptika scheinen schlafanstoßend zu wirken, die Gesamtschlafzeit zu verlängern und die Schlafeffizienz zu verbessern, sowohl bei Patienten mit als auch ohne Schizophrenie. Eine Ausnahme sei Risperidon, welches diesen Effekt nicht zeige. Die tieferen erholsamen Schlafphasen würden durch Olanzapin und Ziprasidon gesteigert; Clozapin habe einen konträren Effekt (Cohrs 2008, S. 954). In Deutschland sind zwei Präparate für die Behandlung primärer Insomnien zugelassen - Melperon und Pipamperon. Sie stammen aus der Gruppe der ,typischen“ Neuroleptika (Riemann et al. 2017).

\subsubsection{Nicht-pharmakologische Maßnahmen zur Förderung des Schlafs im Krankenhaus}

Nicht-pharmakologische Maßnahmen für besseren Schlaf im Krankenhaus nutzen - Knauert und Pisani (2019) zufolge - oftmals eines oder mehrere der folgenden Elemente: Lärm regeln, Licht regeln, Versorgungsroutinen für Patienten ändern, um Ruhepausen zu schaffen, Schlafmasken bereitstellen, Ohrenstöpsel bereitstellen, Schlaf-Schulungen, Angst behandeln, Entspannung fördern und (wenn möglich bzw. zutreffend) Einstellungen von Beatmungsmaschinen anpassen.

Tamrat et al. (2014) untersucht in einer systematischen Übersichtarbeit nicht-pharmakologische Maßnahmen zur Verbesserung des Schlafs von Patienten auf Normalstationen. Die Arbeit umfasst 13 Interventionsstudien mit 1154 Teilnehmern. Die Arten der Interventionen teilten sie ein in (1) Entspannungstechniken (z. B. Massage, eine Art von Geräusch oder Musik, Aromatherapie, ein warmes Getränk oder Kombinationen davon), (2) Interventionen, um Schlafunterbrechungen zu vermindern, indem nächtliche Ruhezeiten geschaffen werden oder eine bessere Schlafroutine der Patienten unterstützt wird und (3) Lichttherapie mit hellem Licht tagsüber, um den nächtlichen Schlaf zu verbessern. In der Arbeit wurden Studien eingeschlossen, die den Schlaf anhand objektiver und subjektiver Maße über die Schlafquantität beurteilten bzw. anhand subjektiver Maße über die Schlafqualität. Die Stärke der Evidenz für eine Verbesserung dieser Endpunkte ist bei Studien über Entspannungstechniken und Lichttherapie niedrig. Die Autoren erklären, dass der Effekt einiger der untersuchten Entspannungstechniken darauf beruhen könnte, dass der mit Schlafproblemen einhergehenden Aktivierung des sympathischen Nervensystems durch die Entspannung entgegengewirkt werde, was den Patienten helfe, zur Ruhe zu kommen. Der Gedanke hinter der Lichttherapie erscheine ebenfalls logisch; helles Licht könne helfen, kurze Schlafeinheiten am Nachmittag zu vermeiden um den nächtlichen Schlaf zu begünstigen. Die Stärke der Evidenz für eine Verbesserung der Schlafquantität und -qualität durch Interventionen zur Minderung der Schlafunterbrechungen und Förderung der Schlafhygiene sei jedoch nicht ausreichend, da qualitativ hochwertige Studien fehlen. Tamrat et al. (2014) schließen mit einer Reihe von Empfehlungen für zukünftige Forschung/Studien. Unter anderem sei ein standardisiertes Protokoll erforderlich, das Unterbrechungen des Schlafs von Patienten minimiere. Es sei außerdem wichtig für Behandelnde die Schwelle erkennen zu können, wann nicht-pharmakologische und wann pharmakologische Maßnahmen notwendig sind. 
Hu et al. (2015) untersuchten in einer Cochrane-Übersichtsarbeit den Effekt nicht-pharmakologischer Interventionen zur Förderung des Schlafs auf Intensivstationen. Die Arbeit umfasst 30 Studien mit 1569 Teilnehmern. Die Interventionen werden von den Autoren folgendermaßen unterteilt: (1) (Kognitive oder Verhaltens-) Psychologische Interventionen, (2) Komplementäre Therapien (z. B. Musiktherapie, Aromatherapie, Massage, geführtes Visualisieren, Akupressur), (3) die Umgebung betreffende Interventionen (z. B. Abgleich der Aktivitäten auf der Intensivstation mit dem Tageslicht, Geräuschreduktion), (4) Soziale Interventionen (z. B. Unterstützung durch die Familie) und (5) Veränderungen der Gerätschaft (z. B. Optimierung der Beatmungsmodi oder der Beatmungsform). Die Endpunkte enthielten Auswirkungen auf den Schlaf, die objektiv gemessen werden konnten (beispielsweise durch Polysomnographie - einer Messung der Körperfunktionen während des Schlafs, die eine Erfassung der Schlafphasen ermöglicht (Dirnberger 2020)) - subjektive Änderungen der Schlafqualität und -quantität (gemessen an Teilnehmerberichten oder Beobachtungen der Pfleger), Risiko für ein Delir während des Aufenthalts, Zufriedenheit der Teilnehmer, Länge des Intensivstationsaufenthalts und Unerwünschte Ereignisse.

Ähnlich wie in der vorher genannten Übersichtsarbeit werden auch in dieser Arbeit die Effekte der Interventionen auf alle Endpunkte als niedrig oder sehr niedrig eingestuft. Der Effekt in der Veränderung der objektiven Schlafvariablen wird als sehr niedrig eingestuft; in der Veränderung der subjektiven Schlafqualität als niedrig. Für die insgesamt niedrige Evidenz der Effekte nennen Hu et al. (2015) zahlreiche Gründe, u. a. das Fehlen einer „Verblindung“ der Teilnehmer, ein hohes Risiko für Verzerrungen in der Zuteilung zu Therapien und das Fehlen von methodischen und statistischen Informationen. Die Ergebnisse weisen allerdings darauf hin - so die Autoren -, dass der Einsatz von Ohrenstöpseln und Schlafmasken möglicherweise einen vorteilhaften Effekt auf die Schlafförderung haben und eventuell das Risiko für ein Delir bei Intensivstationspatienten senkt. Eine weitere Schlussfolgerung betrifft verschiedene Einstellungen der Beatmungsmaschinen, die Schlafquantität, -qualität oder beides bei Intensivstationspatienten verbessern könnten. Hu et al. (2015) betonen den Bedarf an Studien über Musikinterventionen und weiteren Methoden, die auch außerhalb der Intensivstation eingesetzt werden können, wie Massage, Akupressur, die Umgebung betreffende Interventionen, Verhaltenstherapie und psychologische Unterstützung.

Fillary et al. (2015) untersuchten in einem systematischen Überblick Maßnahmen, die den nächtlichen Lärmpegel auf Normalstationen reduzieren sollen. Die Autoren konnten 51 Studien in ihre Übersicht aufnehmen. Im Vergleich zu Studien auf Spezialstationen (wie etwa Intensivstationen) gäbe es einen großen Mangel an Evidenz für effektive Interventionen, um Störungen durch nächtlichen Lärm zu reduzieren. Die verfügbare Evidenz favorisiere einen Lösungsansatz auf drei Ebenen: dem Personal, der Umgebung und der Organisation der Versorgung. Für das Personal solle es designierte Orte geben, an denen laut gesprochen werden kann, und designierte Zeiten, zu denen es ruhiger sein soll. Die Umgebung könne verbessert werden durch geräuschabsorbierende Deckenplatten, regelmäßige Instandhaltung 
der Einrichtungsgegenstände und durch Geräuschmaskierung, in der ein überdeckendes Geräusch, welches einen konstanten, wenig ablenkenden Ton hat, abgespielt wird. Die Organisation der Versorgung könne angepasst werden, indem die Versorgung der Patienten an deren Schlafmuster abgestimmt werde, durch Abendrunden mit der Intention zur Schlafanregung und durch die Versorgung der Patienten mit Ohrenstöpseln und Schlafmasken.

Zusammenfassend wurden viele nicht-pharmakologische Maßnahmen überlegt, entwickelt und umgesetzt, um Patienten zu besserem Schlaf im Krankenhaus zu verhelfen. Welche davon einen Nutzen haben, ist noch nicht ausreichend geklärt. Autoren der Studien äußern den Bedarf für weitere Forschung. Erste Wahl für die Behandlung von Insomnien bei Erwachsenen jedes Lebensalters sollte die kognitive Verhaltenstherapie sein, so die Empfehlung der S3-Leitlinie „Nicht erholsamer Schlaf / Schlafstörungen“ (Riemann et al. 2017). Ansätze zur Umsetzung dieser Empfehlung im Krankenhaus sind wünschenswert, bei vorübergehenden Schlafstörungen wahrscheinlich kaum realisierbar.

\subsubsection{Hypnotika bei älteren Patienten}

Veränderungen im Organismus, die mit steigendem Alter stattfinden, haben Auswirkungen auf die Art und Weise, wie der Körper mit Medikamenten interagiert.

Viele Medikamente - aber nicht alle - wirken mit zunehmendem Alter länger und stärker. Die Wirkung von Medikamenten ist weniger leicht einzuschätzen und zu kontrollieren. Unerwünschte Arzneimittelwirkungen sind bei älteren Patienten entsprechend häufiger und intensiver, sodass Benzodiazepine stärker sedierend wirken können und häufiger Verwirrtheit, Ataxie und Immobilität verursachen. Trizyklische Antidepressiva können Auslöser sein von Agitation, Verwirrtheit, Einschränkungen der Aufmerksamkeit und des Gedächtnisses und Delir. Diese Veränderungen kognitiver Funktionen durch Medikamente können zugleich Anlass für soziale Isolation und dem Rückzug aus der Gesellschaft sein (Turnheim 2003).

Der Einsatz von Benzodiazepinen erhöht das Risiko für Stürze und Hüftfrakturen (Allain et al. 2005). Der Gedanke, dass die neueren Z-Substanzen in Hinsicht auf diese Vorfälle eine sicherere Alternative darstellen, konnte nicht bestätigt werden. Die Häufigkeit von nichtvertebralen Frakturen und Dislokationen bei Patienten mit Zolpidem war in einer Kohortenstudie sogar höher als bei Patienten mit den Benzodiazepinen Alprazolam und Lorazepam und ähnlich wie bei Patienten mit Diazepam (Finkle et al. 2011). Für Patienten in einem deutschen Krankenhaus konnten Wedmann et al. (2019) eine Assoziation mit einem um 2bis 3-fach erhöhten Sturzrisiko feststellen durch sowohl langwirksame Benzodiazepine als auch Z-Substanzen.

Der Zusammenhang zwischen der Behandlung mit Hypnotika und dem Auftreten von Demenz ist ein weiteres Forschungsthema. Die Studienlage hierzu ist nicht einheitlich. In Frankreich untersuchten Gage et al. (2012) den Zusammenhang zwischen Demenz und Benzodiazepin-Gebrauch bei älteren Patienten anhand einer prospektiven Kohortenstudie. Die 1063 Teilnehmer hatten anfangs der Studie keine Demenz-Diagnose und nahmen bis mindestens 
ins dritte Jahr der Folge-Untersuchungen keine Benzodiazepine ein. Am Ende der Folgeuntersuchungen, nach 15 Jahren, konnten 253 neue Demenz-Fälle festgestellt werden. Die neu begonnene Einnahme von Benzodiazepinen war assoziiert mit einem um ungefähr $50 \%$ erhöhten Risiko, an Demenz zu erkranken.

Dass Patienten einen vermehrten Benzodiazepingebrauch zeigen, da sie an demenziell bedingten psychischen Problemen leiden, stellt eine mögliche umgekehrte Kausalität dar. Gage et al. (2012) können trotz eines Studiendesigns, bei dem dieses Problem bedacht wurde, einen solchen Zusammenhang nicht gänzlich ausschließen.

$\mathrm{Zu}$ gegensätzlichen Ergebnissen kamen Gray et al. (2016), die in den USA ebenfalls eine Kohortenstudie durchführten über den Gebrauch von Benzodiazepinen bei älteren Patienten und dem Risiko neu auftretender Demenz oder dem Nachlassen kognitiver Fähigkeiten. Die Studie umfasste 3434 Teilnehmer, 65 Jahre oder älter, ohne Demenz am Anfang der Untersuchung. Bis zur Folge-Untersuchung (durchschnittlich 7,3 Jahre später) entwickelten 797 der Teilnehmer eine Demenz. In dieser Untersuchung konnte allerdings kein Zusammenhang zwischen der Einnahme von Benzodiazepinen und dem Auftreten einer Demenz gefunden werden.

Mit Blick auf die heterogene Studienlage kommen Pariente et al. (2016) zu folgendem Schluss: Der rationale Gebrauch - nicht länger als drei Monate - führe nicht zu einem erhöhten Risiko von Demenz. Für den Langzeitgebrauch, für den bisher kein Nutzen gezeigt werden konnte, dürfte ein erhöhtes Demenzrisiko nicht ignoriert werden - vor allem da eine kausale Assoziation nach dem bisherigen Wissensstand nicht ausgeschlossen werden konnte.

Für Medikamente mit anticholinergen Eigenschaften, darunter auch einige schlafanstoßende Antidepressiva und Antipsychotika, wurde in einer aktuellen Fall-Kontroll-Studie ein erhöhtes Risiko für Demenz festgestellt (Coupland et al. 2019). Verglichen wurden in der Studie knapp 60000 Patienten mit rund 226000 Kontrollpatienten ohne Demenz. Das Demenzrisiko war schon bei geringster Medikamentendosis um fast 50\% erhöht. Die Autoren betonen, dass die Beobachtungsstudie keine kausalen Zusammenhänge liefere. Die Ergebnisse würden allerdings Ergebnissen weiterer Studien zu dem Thema entsprechen.

Glass et al. (2005) führten eine Meta-Analyse durch über Risiken und Nutzen von Hypnotika bei älteren Patienten mit Schlafstörungen. Sie konnten 24 randomisierte kontrollierte Studien mit 2417 Teilnehmern in der Analyse einschließen, die eine Hypnotika-Behandlung mit Placebo oder einem anderen aktiven Vergleichspräparat verglichen. Der Nutzen wurde gemessen anhand subjektiver Beobachtungen der Patienten über Veränderungen ihres Schlafs. Variablen waren Schlafqualität, Gesamtschlafzeit, Schlaflatenzzeit, Mühelosigkeit einzuschlafen und Häufigkeit nächtlichen Erwachens. Untersuchte Risiken waren unerwünschte kognitive Wirkungen (Gedächtnisverlust, Verwirrtheit, Orientierungsverlust), unerwünschte psychomotorische Wirkungen (Mitteilungen über Schwindel, Verlust von Gleichgewicht oder Stürze) und Hangover-Effekte am Morgen nach der Schlafmittelgabe. Der Gebrauch von 
Hypnotika wirkte sich positiv auf die subjektiven Schlafvariablen aus: Sowohl die Gesamtschlafzeit als auch die nächtlichen Schlafunterbrechungen waren mit Hypnotika-Behandlung besser als mit Placebo. Die Effektgröße war allerdings gering. Für unerwünschte Wirkungen war das Risiko in der Patientengruppe mit Hypnotika erhöht, auch wenn sie zumeist reversibel und nicht ernsthaft waren, wie Müdigkeit, Schlappheit, Kopfschmerzen und vermehrte Tagesmüdigkeit. Ernsthaftere Vorfälle waren Stürze und Kraftfahrzeugunfälle.

Die Autoren argumentieren, dass die Nachteile beim Einsatz von Hypnotika überwiegen. Damit 1 Patient von der Behandlung mit einem Hypnotikum profitiert, müssen 13 behandelt werden; damit 1 Patient eine der unerwünschten Nebenwirkungen erfährt, müssen nur 6 Patienten behandelt werden. Das Risiko einer unerwünschten Wirkung ist also ungefähr doppelt so hoch wie die Chance einer erwünschten Wirkung.

Nicht zuletzt scheint die Behandlung mit Hypnotika einen Einfluss auf die Mortalität zu haben. Kripke et al. (2012) untersuchten dies in einer longitudinalen Beobachtungsstudie, für die sie elektronische Patientenunterlagen von knapp 11000 Patienten mit und 24000 ohne Hypnotika aus den Jahren 2002 bis 2007 auswerteten; der Beobachtungszeitraum war im Durchschnitt 2,5 Jahre. Patienten mit einer Hypnotika-Verordnung hatten ein 4,6-mal höheres Risiko zu sterben als Patienten ohne Hypnotika-Verordnung. Die Risiken der Hypnotika traten in jeder Altersgruppe auf, die Sterberisiken waren jedoch in der Gruppe der Jüngeren (18 bis 55 Jahre) höher als bei den Älteren (Kripke et al. 2012, S. 4).

Die Datenlage, die bei älteren Patienten eher gegen den Gebrauch von Benzodiazepinen zur Behandlung von Schlafstörungen spricht, führte zu entsprechenden Empfehlungen über den Gebrauch von Hypnotika bei älteren Patienten seitens der Fachgesellschaften (American Geriatrics Society 2015; Canadian Geriatrics Society 2017). Die Empfehlung der Deutschen Gesellschaft für Geriatrie lautet: „Benzodiazepine oder andere Sedativa beziehungsweise Hypnotika bei älteren Patienten sollen nicht als Mittel der ersten Wahl im Falle von Schlafstörungen, Agitation oder Delir eingesetzt werden“ (Gogol 2016). 


\subsection{Interventionen zur Verbesserung potenziell inadäquater Medikation}

Um die Nutzung schlafanstoßender Medikamente zu verringern bzw. sie gezielter und angemessener einzusetzen, wurden bisher vielerlei Maßnahmen ausprobiert. Wilson et al. (2018) testeten bei hospitalisierten Patienten eine relativ „simple“ Herangehensweise: Patienten, die chronisch oder regelmäßig Benzodiazepine einnahmen, erhielten eine Informationsbroschüre über Schlafmittel und wurden ermutigt, mit ihrem behandelnden Team zu sprechen, falls sie die Medikamente absetzen wollten. Immerhin setzten 60\% der Patienten ihre Benzodiazepine ab; im Jahr vorher, in dem keine Intervention stattfand, taten dies nur rund $20 \%$. Eine Übersichtsarbeit von Mugunthan et al. (2011) bestätigt den Effekt ähnlicher MinimalInterventionen. Einfache Maßnahmen wie z. B. Briefe an zu Hause lebende Patienten können offensichtlich den Gebrauch selbst langfristig verschriebener Benzodiazepine reduzieren.

Ein Beispiel für eine Intervention, die auf mehreren Ebenen stattfand, ist das Programm von Chung et al. (2018), das Strukturen im Krankenhaus änderte und Informationen an Behandelnde und Patienten bereitstellte. Zielgruppe waren alle Patienten, die Schlafmittel erhielten. Gesundheits- und Krankenpfleger, die in Schlafhygiene geschult wurden, wurden in jeder Station eingesetzt. Zusätzlich wurden Ärzte und Pfleger zur richtigen Verordnung und dem Absetzen von Schlafmitteln geschult. Nach der Intervention reduzierte sich der Anteil der Patienten, die Schlafmittel bis zum Zeitpunkt der Entlassung erhielten. In einer anderen Intervention involvierten Badr et al. (2018) Krankenhausapotheker, die das Absetzen von Schlafmitteln während Patientenvisiten oder über ein Telefonat mit dem zuständigen Arzt empfehlen konnten. Die Anzahl neuer Schlafmittel-Verschreibungen war nach der Intervention um 25\% reduziert, verglichen mit der Kontrollgruppe. Ebenfalls verringerte sich der gleichzeitige Gebrauch mehrerer Schlafmittel.

Ob bisherige stattgefundene Interventionen tatsächlich effektiv sind, wird in einer CochraneÜbersichtsarbeit anhand von 32 Studien untersucht (Rankin et al. 2018). Die Arbeit untersucht Interventionen, die zur Verbesserung der Polypharmazie bei älteren Menschen führen sollten, also der Verbesserung der Behandlung mit mehreren Medikamenten gleichzeitig. Der Einsatz „Potenziell inadäquater Medikamente“ (ein Thema der vorliegenden Arbeit) wird von den Autoren als eine Ursache für unangemessene Polypharmazie betrachtet. Entsprechend wird untersucht, ob Interventionen zu einem Rückgang potenziell inadäquater Medikation führen konnten.

Einige Verbesserungen konnten in Endpunkten wie der Anzahl potenziell inadäquater Verschreibungen und der Anzahl potenziell unterlassener Verschreibungen gesehen werden. In anderen Endpunkten über inadäquate Medikation bestand ein Mangel an Gewissheit, so etwa in der Menge der potenziell inadäquaten Medikamente, die ein Patient erhielt, dem Anteil der Patienten mit einem oder mehreren potenziell inadäquaten Medikamenten und dem Anteil der Patienten mit einer oder mehreren potenziell unterlassenen Verschreibungen. Ansätze 
erfolgreicher Interventionen beruhen der Studie zufolge u. a. auf multidisziplinärer Beteiligung, z. B. durch die aktive Beteiligung von Geriatern, und dem Einsatz von Clinical Decision Support Systemen.

Welche Elemente einer Intervention zu Erfolgen in der Verbesserung angemessener Polypharmazie führen, bleibe, so schlussfolgern die Autoren, letztlich unklar. Fragen, die unbeantwortet bleiben, sind beispielsweise, ob Interventionen innerhalb eines einzelnen Zeitabschnitts ausreichend seien oder ob Patienten den Interventionen wiederholt exponiert sein sollten; ob Interventionen vielschichtig sein sollten oder „einfach“ und wie lange eine Intervention dauern sollte.

Tipps für zukünftige Studien, die Rankin et al. (2018) geben, sind: (1) Sich an der Richtlinie des Medical Research Council über das Design komplexer Interventionen zu orientieren. (2) Die Entwicklung, den Inhalt, sowie den Hintergrund der Träger der Intervention genau zu dokumentieren. (3) Qualitative Elemente einzubauen, die hilfreich sein können um Einblicke in Veränderungsprozesse zu erhalten, die der Intervention unterliegen. (4) Details über den Kontext der Intervention zu verstehen und mitzuteilen und (5) eine ausreichend lange Dauer von Follow-Up-Untersuchungen einzuplanen. Abschließend nennen Rankin et al. (2018) als vielleicht wichtigsten Punkt für zukünftige Studien (6) klinische und auf den Menschen bezogene Endpunkte, die für ältere Patienten relevant sind, auszuwählen, wie z. B. Krankenhausaufnahmen und unerwünschte Arzneimittelereignisse.

Eine weitere Cochrane-Übersichtsarbeit untersuchte anhand von zwölf Studien Interventionen zur Optimierung von Verordnungen bei älteren Patienten in Pflegeheimen (Alldred et al. 2016). Fünf der Studien weisen darauf hin, dass die Angemessenheit von Medikamenten durch mehrschichtige Interventionen verbessert werden konnte. Inwiefern diese Interventionen letztlich auch die Gesundheit der Bewohner verbessern, sei nicht klar.

Zusammenfassend lässt sich feststellen: Erfolgreiche Interventionen für die Verbesserung der medikamentösen Versorgung älterer Patienten involvierten mehrere Berufsgruppen und den Einsatz von Clinical Decision Support Systemen. Aufgrund mangelnder Qualität der Studien ist die Frage, ob Interventionen die Medikation älterer Patienten verbessern und welche Elemente der Interventionen entscheidend sind, noch nicht beantwortet. Um offene Fragen zu klären, sind randomisierte kontrollierte Studien von hoher Qualität mit einer angemessenen statistischen power, idealerweise im Cluster-Design, notwendig. 


\subsection{Screening-Tools für potenziell inadäquate Medikation}

Im folgenden Kapitel sollen verschiedene Listen potenziell inadäquater Medikamente betrachtet werden. Sie kommen oftmals in Interventionen zum Einsatz mit dem Ziel, die medikamentöse Versorgung von Patienten zu verbessern; so auch in einigen der in Kapitel 2.2 ausgeführten Interventionen. Anhand der Listen können Behandelnde potenziell inadäquate Medikamente entdecken (Screening-Tools), um dann therapeutische Alternativen einzusetzen. Solche Listen können eingeteilt werden in explizite Tools (Kriterien-basiert) oder implizite Tools (Ermessens-basiert) (O’Connor et al. 2012, S. 439). Explizite Kriterien haben den Vorteil, einfach und effektiv interpretiert werden zu können (Stewart et al. 2017). Die Entwicklung erfolgt durch Literaturrecherche, Expertenmeinungen und Konsens-Prozesse (Rankin et al. 2018, S. 8). So wurde die im Jahr 2011 veröffentlichte PRISCUS-Liste (priscus, lat. ,altehrwürdig“) mit Hilfe einer Literaturrecherche, einer qualitativen Analyse anderer Listen mit potenziell inadäquaten Medikamenten (PIM) und einer darauffolgenden strukturierten Expertenbefragung erarbeitet und stellt ein explizites Screening-Tool dar (Holt et al. 2010). Sie wurde für den deutschen Arzneimittelmarkt entworfen.

Neben der Auflistung von PIM macht sie den Behandelnden anhand von Therapiealternativen Vorschläge, wie die potenziell inadäquaten Medikamente ersetzt werden können. Die Liste umfasst 83 verschiedene Wirkstoffe aus 18 Wirkstoffgruppen. Als potenziell inadäquate schlafanstoßende Medikamente werden Wirkstoffe aus den Gruppen Benzodiazepine und Z-Substanzen genannt. Die Einstufung dieser Medikamente als inadäquat erfolgt aufgrund unerwünschter Arzneimittelereignisse wie Stürzen (einhergehend mit einem höheren Frakturrisiko), paradoxer psychischer Reaktionen und kognitiver Funktionseinschränkungen. Als pharmakologische Alternativen werden Baldrian, sedierende Antidepressiva wie Mirtazapin und kurzwirksame Z-Substanzen in einer niedrigen Dosierung empfohlen. Nicht-pharmakologisch wird zur Schlafhygiene geraten (vgl. Neukirchen 2018, S. 6). Nach diesen Empfehlungen wurde auch die im vorliegenden Projekt zum Einsatz gekommene Standard Operating Procedure (SOP) ,Schlafstörungen im Krankenhaus“ entworfen und an die Mitarbeiter im Evangelischen Krankenhaus Weende verteilt.

Die PRISCUS-Liste reiht sich in eine Vielzahl internationaler PIM-Listen für ältere Menschen ein (O’Connor et al. 2012). Bekanntester und ältester Vertreter ist die Beers-Liste, die vor fast 30 Jahren veröffentlich wurde (Beers et al. 1991). Siebert et al. (2013) vergleichen in ihrer Studie die PRISCUS-Liste mit der Beers-Liste und den START/STOPP-Kriterien und stellen so die jeweiligen Stärken und Schwächen dar.

Ein Vorteil der PRISCUS-Liste sei demnach, dass sie an den deutschen Arzneimittelmarkt angepasst ist und folglich eine größere Anzahl von PIM erfasse als die Beers-Liste. Auch das Aufzeigen therapeutischer Alternativen zu PIMs sei ein Merkmal, das andere Listen, wie die Beers-Liste nicht aufweisen. Medikamente werden auf der PRISCUS-Liste dosisabhängig differenziert beurteilt. Von Vorteil sei dies beispielsweise bei Benzodiazepinen, deren Einsatz bei psychischen Krankheiten unumgehbar sein kann (Siebert et al. 2013). Zusätzlich schlägt 
die Liste Maßnahmen vor, falls ein PIM-Arzneimittel trotz allem verwendet werden soll (z. B. therapeutisches Drug-Monitoring).

Ein Nachteil, der durch die Anpassung an den deutschen Arzneimittelmarkt entsteht, ist die begrenzte Übertragbarkeit der Liste auf andere Länder. Um auf dieses Problem einzugehen entwarfen Renom-Guiteras et al. (2015) eine für ganz Europa geltende Liste. Andere Nachteile, die Siebert et al. (2013) nennen, seien die eingeschränkte klinische Relevanz im Vergleich zur Beers-Liste, bei der in einer zweiten Tabelle die Wirkstoffe auf eine Krankheit oder ein Syndrom bezogen aufgelistet sind. Die Autoren empfehlen deswegen eine Ergänzung der PRISCUS-Liste um eine diagnoseabhängige Einschätzung der Wirkstoffe. Ein weiterer Kritikpunkt sei die explizite Auflistung bestimmter Arzneistoffe. So könne von Behandelnden z. B. fälschlicherweise angenommen werden, dass einzelne nichtgelistete Arzneistoffe, die zu einer inadäquaten Medikamentengruppe gehören, bei älteren Patienten geeignet wären. Die STOPP-Kriterien würden dieses Problem durch den Verzicht auf das Nennen einzelner Arzneistoffe zugunsten von Medikamentengruppen umgehen (O’Mahony et al. 2015). So sei keine wiederholte Anpassung an aktuelle Marktgegebenheiten notwendig und die Kriterien sind international übertragbar (Siebert et al. 2013, S. 37).

Seit der ersten Veröffentlichung 2011 wurde die PRISCUS-Liste bis zum jetzigen Datum (Dezember 2020) nicht mehr aktualisiert. Schon 2002 schrieben Gurwitz und Rochon, dass die Beers-Liste mit „Reliquien einer vergangenen Pharmakopöe“ bestückt sei, worauf sie sich auf den Wirkstoff Flurazepam beziehen, welcher auch in der PRISCUS-Liste vorkommt (Gurwitz und Rochon 2002). Immerhin wurde die Beers-Liste seit der Veröffentlichung bereits mehrmals aktualisiert und wird derzeit in 3-Jahres-Abständen von der American Geriatrics Society geupdatet (Beers 1997; Fick et al. 2003; The American Geriatrics Society 2012 Beers Criteria Update Expert Panel 2012; The American Geriatrics Society 2015 Beers Criteria Update Expert Panel 2015; The American Geriatrics Society 2019 Beers Criteria Update Expert Panel 2019).

Ob der Einsatz von Medikamenten aus der PRISCUS-Liste aus ökonomischer Sicht sinnvoll ist, untersuchten Pohl-Dernick et al. (2016). Sie verglichen die Verschreibungskosten der 30 am häufigsten verordneten PIM mit den Kosten, die durch die Verschreibung äquivalenter Wirkstoffe der PRISCUS-Liste verursacht würden (Endres et al. 2018). Die Ergebnisse zeigen, dass der Ersatz mit PRISCUS-Medikamenten zu einem Kostenanstieg für die Krankenversicherungen führen würde. Kurzfristig sei es für die Krankenversicherungen dementsprechend nicht von Anreiz, einen Austausch der Medikamente zu fördern. Längerfristig allerdings sei zu bedenken, dass die Behandlung mit PIM-Hypnotika mit einem erhöhten Risiko für kostenaufwendige Stürze assoziiert ist (Stockl et al. 2010). Auch höhere Hospitalisierungsraten und wiederholte Arztbesuche müssen einkalkuliert werden, um den Kosten-Nutzen-Effekt des Einsatzes von PIM-Alternativen abschließend zu beurteilen. 
PIM-Listen stellen ein Tool dar, das Ärzte für die Verbesserung der Patientenversorgung nutzen können. Der Einsatz solcher Listen ist in bisherigen papierbasierten Verordnungssystemen ein zusätzlicher Arbeitsschritt. O'Connor et al. (2012) sowie Gurwitz und Rochon (2002) sprechen sich für die Notwendigkeit automatisierter elektronischer Arzneiverordnungssysteme (Computerised Physician Order Entry System with Clinical Decision Support) aus, in denen potenziell inadäquate Verordnungen zum Zeitpunkt der Verordnung festgestellt und direkt zurückgemeldet werden. Solche CPOE-CDS-Systeme sind in deutschen Krankenhäusern bisher noch nicht flächendeckend verbreitet (Kern 2018, S. 274). Ein fortschreitender Wechsel zu elektronischen Datenverarbeitungssystemen in Krankenhäusern lässt die Implementation dieser Systeme, angereichert mit aktuellen PIM-Informationen, allerdings als möglich und machbar erscheinen. Eine Übersichtsarbeit bestätigt, dass Computerinterventionen die Anzahl potenziell inadäquater Medikation reduzieren können (Dalton et al. 2018).

\subsection{Erfolg bisheriger Interventionsstudien}

Interventionsstudien mit dem Ziel, den Einsatz von Hypnotika und Sedativa zu reduzieren bzw. gezielter, also evidenzbasierter zu gestalten, waren teilweise erfolgreich, teilweise nicht. So konnten Badr et al. (2018) in einer kontrollierten Krankenhaus-Intervention mit 197 Patienten (100 Kontrolle, 97 Intervention) die Anzahl der Patienten, die mehr als ein Sedativum bzw. Hypnotikum zur gleichen Zeit erhielten, erfolgreich von 34\% auf $16 \%$ reduzieren. Dolan et al. (2012) untersuchten, ob Briefe an Hausärzte mit Informationen über ihr Verschreibungsverhalten und über Benzodiazepine und Z-Substanzen etwas an der Menge der Verordnungen ändern würde. Der beobachtete Rückgang der Verordnungen von 54\% auf 46\% war nicht-signifikant. Elliott et al. (2001) gaben Ärzten, Apothekern und Pflegern in 9 Krankenhäusern Rückmeldungen über ihre Benzodiazepin-Verordnungen. Nach diesem Feedback stieg die Angemessenheit der Verordnungen signifikant an (20\% auf 44\%); die Anzahl der Verordnungen konnte allerdings nicht signifikant gesenkt werden (36\% auf 31\%). Pimlott et al. (2003) gaben Hausärzten Rückmeldungen über ihre Benzodiazepin-Verordnungen und Fachinformationen über das Thema. Die anschließende Reduktion der BenzodiazepinVerordnungen stellte sich als nicht-signifikant heraus.

In ihrem nicht-systematischen Review über den Erfolg von Interventionen der letzten 20 Jahre zur Verbesserung des Benzodiazepin-Gebrauchs schildern Smith und Tett (2010), dass viele verschiedene Herangehensweisen erprobt wurden - mit schwankendem Erfolg. Einen Hinweis auf mögliche Ursachen liefern Rankin et al. (2018) in ihrer Cochrane-Übersichtsarbeit: Die Anzahl der Interventionsstudien zu dem Thema Polypharmazie bei älteren Patienten sei gestiegen - viele der Studien seien aber durch eine geringe Stichprobenzahl und geringer Gewissheit der Evidenz limitiert. 
Erfolgsversprechend scheinen am ehesten Studien zu sein, die auf mehreren Ebenen intervenieren (Smith und Tett 2010; Mostofian et al. 2015; Rankin et al. 2018). Dieser Ansatz auf mehreren Ebenen - auch Multi-level-Ansatz oder komplexe Intervention genannt - kann heißen, dass die Intervention auf mehrere Zielgruppen abzielt, dass die Intervention mehrere Angriffspunkte anstrebt oder beides (Smith und Tett 2010).

\subsection{Interventionsstudien in Deutschland: ein weißer Fleck}

Probleme bei Ein- und Durchschlafen sind Herausforderungen im Krankenhaus: für Patienten, aber auch für die Nachtschicht. Der großzügige Einsatz von schlafanstoßenden Medikamenten ist oberflächlich eine Lösung, letztlich aber ein Problem vor allem für ältere Patienten.

In deutschen Krankenhäusern gab es unseres Wissens keine Versuche, daran etwas nachhaltig zu ändern. Die meisten Versuche, also Interventionsstudien, sind aus Ländern wie den USA und Kanada. Die Krankenhauskultur und Gesundheitssysteme anderer Länder können sich allerdings deutlich unterscheiden. Erst entsprechende Mehrebenen-Interventionen in deutschen Krankenhäusern könnten Auskunft darüber geben, ob sie auch hier zu einer Reduktion des Gebrauchs schlafanstoßender Medikamente führen. 


\section{Forschungsfragen und Ziel der Studie}

Ziel der Studie war es, festzustellen, ob eine auf mehreren Ebenen stattgefundene Intervention zu einem rationaleren Einsatz schlafanstoßender Medikamente bei älteren Patienten führte. Dafür wurden zwei Zeiträume Juli bis Mitte August 2013 (vor der Intervention) und Juli bis Mitte August 2017 (nach der Intervention) verglichen. Die Forschungsfragen lauteten:

1. Wirkte sich die Intervention auf die Menge der verabreichten schlafanstoßenden Medikamente aus?

2. Wirkte sich die Interventionen auf die Angemessenheit der verabreichten schlafanstoßenden Medikamente aus? 


\section{$4 \quad$ Methoden}

\subsection{Studiendesign}

Es handelt sich um eine nicht-randomisierte, prospektive, offene Studie mit einem Prä-PostDesign mit Kontrollgruppe, also ein Quasi-Experiment (Hertel et al. 2010). Die Studie beruht auf Routinedaten (einem sog. Chart Review) eines Krankenhauses, die zu zwei Zeiträumen (vor und nach Intervention) ausgewertet wurden.

\subsection{Kontext der Arbeit}

Ein vom Bundesgesundheitsministerium gefördertes Projekt $(, \ldots$ da gab es so wunderbare Schlaftabletten - Verordnungen von Benzodiazepinen und Z-Substanzen an der Schnittstelle von Krankenhaus und Hausarzt") untersuchte den Umgang mit Schlaf- und Beruhigungsmitteln im hausärztlichen und im stationären Umfeld. Aus den Ergebnissen der Untersuchungen entstand die Initiative „Schlaffreundliches Krankenhaus“ mit dem Ziel, die Schlafqualität im Krankenhaus zu verbessern und die Menge an Schlafmitteln zu reduzieren (Heinemann und Weiß 2016).

Die Planung und Durchführung des Projektes erfolgte von April 2014 bis Juni 2017 in Zusammenarbeit zwischen dem Evangelischen Krankenhaus Göttingen-Weende und dem Institut für Allgemeinmedizin der Universitätsmedizin Göttingen. Das Projekt verlief in 2 Phasen. Phase 1 war eine Bestandsaufnahme mittels eines Mixed-Methods Ansatzes. Durch standardisierte Befragung des Krankenhauspersonals und von Patienten, Chart Reviews (Auswertung von Krankenakten) und qualitativen Interviews mit den Hauptbeteiligten sollte die Verordnung von Schlaf- und Beruhigungsmitteln im Krankenhaus in Praxen und an den Schnittstellen aus verschiedenen Perspektiven erhoben und analysiert werden (Heinemann et al. 2016). Die Ergebnisse waren Grundlage für die in Phase 2 durchgeführten Fokusgruppen aus Krankenhausärzten, Pflegepersonal und Hausärzten. Dort wurden Ideen gesammelt, wie man den Verbrauch von Schlaf- und Beruhigungsmitteln verringern könnte und wie man gemeinsam mit Patienten über das Thema reden könnte. Aus den Ideen wurden Maßnahmen entwickelt, die erstmalig mit der Initiative Schlaffreundliches Krankenhaus im Evangelischen Krankenhaus Göttingen-Weende umgesetzt wurden. Die Maßnahmen richteten sich an Patienten und das Personal.

Maßnahmen für Patienten:

- Gezielte Informationen

(z. B. durch die Website www.schlaffreundliches-krankenhaus.de, Poster)

- Bereitstellung von „Hausmitteln“ (Schlafbrille, Tee, Ohrstöpsel). 
Maßnahmen für das medizinische und pflegerische Personal:

- Handlungsempfehlungen für neu aufgenommene Patienten

- Verordnungshilfe (Leitlinie) für schlafanstoßende Mittel (Präparate, Dosierung, Alternativen)

- Schulungen für alle Berufsgruppen, inkl. Auszubildende.

Die hier stichwortartig genannten Maßnahmen des Projekts waren nicht Gegenstand der vorliegenden Arbeit.

\subsection{Setting}

Die Datenerhebung für die hier vorgelegte Untersuchung fand im Evangelischen Krankenhaus Göttingen-Weende statt.

Das Haus ist ein Krankenhaus der Grund- und Regelversorgung. Das Krankenhaus hatte 2013 zwei Standorte - Göttingen-Weende und Bovenden-Lenglern. Bis 2017 kam ein dritter Standort dazu, Göttingen/Neu-Mariahilf. Die Studie beschränkt sich auf Akten des Standorts Göttingen-Weende, in dem die Intervention stattgefunden hat. In die Erhebung wurden die Fachabteilungen Innere Medizin, Geriatrie, Allgemein- und Viszeralchirurgie, Unfallchirurgie und Orthopädie, Plastische Chirurgie, Urologie und die Hals-Nasen-Ohrenheilkunde einbezogen.

\subsection{Patienten}

Die Grundlage der Untersuchung waren zwei Stichproben, bestehend aus Krankenakten aller Patienten, die zum Zeitpunkt des Aufenthaltes 65 Jahre oder älter waren und jeweils vom 01.07. bis 11.08. in den Jahren 2013 und 2017 für einen stationären Aufenthalt aufgenommen wurden. Die Dauer des Aufenthalts musste über mindestens zwei Tage gehen, um sicherzustellen, dass eine Übernachtung stattgefunden hat.

Ausgeschlossen wurden Patienten, die während des Aufenthaltes verstarben. Wir gingen davon aus, dass in diesen Fällen eine intensivere Betreuung notwendig war, ggf. mit vermehrtem Gebrauch von schlafanstoßenden Mitteln. Ein weiteres Ausschlusskriterium war ein Aufenthalt auf einer Intensivstation, der sich über den kompletten Zeitraum des Krankenhausaufenthalts streckte. Medikamentengaben auf der Intensivstation wurden bei der Erhebung nicht gezählt.

Für die Erhebung mussten wir uns darauf festlegen, „Fälle“ zu bearbeiten, anstatt „Patienten“. Jeder neu aufgenommene Patient galt als neuer Fall, auch wenn er zum wiederholten Male aufgenommen wurde. Ein Patient, der im betreffenden Zeitraum, 01.07. bis 11.08. 2013/2017, mehr als einmal aufgenommen wurde, bildete also mehrere Fälle. GleichermaBen wurde bei einem Stationswechsel während des Aufenthalts eine neue Akte (= neuer Fall) angelegt. Der Einfachheit halber bezeichnen wir die Fälle als Patienten. Tatsächlich kann aber derselbe Patient mehrfach als Fall in der Studie auftauchen. 


\subsection{Datenerhebung}

Die für die Untersuchung bestimmten Informationen wurden aus Akten, die für den Zeitraum 2013 in analoger Form und für den Zeitraum 2017 in digitaler Form vorlagen, in die Dateneingabemaske (s. Kapitel 4.6.2.1) eingetragen (= Chart Review). Wir hielten uns für die Durchführung des Chart Review an die Empfehlungen von Panacek (2007) (Tabelle 1).

Tabelle 1 „Die 10 Gebote für die Durchführung von Aktenauswertungsstudien“ nach Panacek (2007)

1. Definiere die Forschungsfrage prospektiv vor der Datenerhebung.

2. Definiere die Auswahlkriterien prospektiv.

- Detaillierte Ein- und Ausschlusskriterien

3. Definiere Variablen und Richtlinien der Studie prospektiv.

- Diese müssen möglicherweise im Studienverlauf erweitert oder angepasst werden.

4. Sorge für eine hohe Qualität der Erhebenden

- Qualifikation und Training

5. Sorge für Konsistenz in der Erhebung.

- Wie mit einer detaillierten Datenmaske oder einem Computerprogramm

6. „Verblinde“ die Datenabstraktoren über den Zweck der Studie (wenn möglich).

7. Sorge für Qualitätssicherung der Datenerhebung durch regelmäßige Kontrollen.

8. Sorge für Qualitätssicherung der Datenverarbeitung durch Doppelt-Eingaben-Techniken.

9. Sei konsequent im Verlauf.

- Starte von Anfang an, falls es signifikante Änderungen in irgendeiner der Hauptvariablen gibt.

10. Kontrolliere den Fortschritt der Studie.

- Halte regelmäßige Treffen.

Mit freundlicher Genehmigung des Elsevier Verlages

\subsubsection{Datenauswahl}

Folgende Angaben wurden aus den Patientenakten aufgenommen:

- Geburtsjahr

- Geschlecht

- Aufenthaltszeitraum

- Abteilung, klassifiziert in:

- Innere / Chirurgie / Geriatrie

- Art der Aufnahme, klassifiziert in:

- Von Zuhause / Aus Pflegeheim / Aus anderem Krankenhaus / Aus anderer Abteilung / Unbekannt

- Verordnungsstatus von Schlafmitteln zum Zeitpunkt der Aufnahme, klassifiziert in:

○ Dauerverordnung / Bedarfsverordnung / Beides / Nicht klar erkennbar / Nicht vorhanden

- Patient verstorben? 
- Patient gestürzt?

- Intensivstationsaufenthalte während des Aufenthalts

- Diagnosen, Auswahl:

○ Demenz, Schlafstörung, Angst- oder Panikattacke, Delir, Depression, Psychose, Durchgangssyndrom, Abhängigkeit oder Abusus (bezogen auf Hypnotika), andere neurologisch-psychiatrische Diagnosen

- Medikation während des Aufenthalts:

- Art der Verordnung, klassifiziert in:

- Keine / Dauerverordnung / Bedarfsverordnung / Beides / Unbekannt

- Anzahl der Tage, an denen schlafanstoßende Medikamente verabreicht wurden

- Bei PIM: Anzahl der Tage, an denen ein schlafanstoßendes Medikament in einer inadäquaten Dosierung verabreicht wurde

- Tage mit Schlafmittel (Zahl)

Eine der erhobenen Diagnosen war das „Durchgangssyndrom“. Dieser Begriff, der so in den Akten vorkam, wird mittlerweile nicht mehr genutzt. In einem Gespräch mit zwei klinisch tätigen Ärzten wurde die Entscheidung getroffen, diese Diagnosen für die Auswertung mit „Delir“-Diagnosen zusammenzufügen (s. a. Kap. 6.1.4). Während der Erhebung fanden wir Diagnosen, die schlafmittelindizierend sein können aber nicht Teil unserer Diagnosen-Liste waren. Beispiele sind „affektive Störung“ und „Anpassungsstörung“. Bei Patienten mit solchen Diagnosen konnte eine Notiz zu dem jeweiligen Fall gemacht werden. So wurden die Diagnosen gesammelt und im Nachhinein unter bereits vorhandenen Diagnosen eingeordnet oder als ,andere neurologisch-psychiatrische Diagnosen“ eingeordnet.

\subsubsection{Klassifikation der Medikamente nach Wirkstoff}

Der Fokus der Datenerhebung lag darauf, festzustellen, ob und wieviel schlafanstoßende Medikamente im Krankenhaus Weende vor und nach der Intervention verabreicht wurden. Dafür wurde eine Liste der zu erhebenden Wirkstoffe erstellt, orientiert an Benkert und Hippius (2019) (s. Anhang 8.1). Die Liste war nach sedierenden Wirkstoffen aus den Medikamentengruppen Antidepressiva, Antihistaminika, Antipsychotika und Hypnotika geordnet. Sie enthielt sowohl die Wirkstoffnamen (sog. Frei- oder generische Namen) als auch die Markennahmen der Präparate, die das Krankenhaus bestellt hat. Die Präparatenamen, die vom Krankenhaus bestellt wurden, sind zur Einhaltung des Datenschutzes nicht auf der im Anhang vorliegenden Wirkstoffliste. Das genaue Vorgehen beim Erstellen der Wirkstoffliste und das Verfahren, wie Medikamente als schlafanstoßend bewertet wurden, ist im Anhang $8.2 \mathrm{zu}$ finden.

Traditionell zu den schlafanstoßenden Medikamenten gehörend sind Wirkstoffe aus den Gruppen der Benzodiazepine und Z-Substanzen (Steiger et al. 2019). In der Klinik als Schlafmittel eingesetzt werden allerdings auch Medikamente aus anderen Wirkstoffgruppen, wie Neuroleptika und Antidepressiva (Enzensperger und Lehmann 2007). Um ein möglichst breites Feld abzudecken wurden für die vorliegende Dissertation auch Wirkstoffe aus diesen 
weiteren Wirkstoffgruppen erhoben. In Anhang 8.3 ist eine Liste der Wirkstoffe dieser Dissertation mit einem Überblick und Abgleich von sieben weiteren Studien über Schlafmittel.

\subsubsection{Bewertung nach potenziell inadäquater Medikation}

Ältere Menschen haben bei manchen Medikamenten ein höheres Risiko für unerwünschte Arzneimittelwirkungen. Die PRISCUS-Liste (Holt et al. 2011) stellt diese potenziell inadäquate Medikation (PIM) dar und nennt Therapiealternativen. Ein Ziel der Interventionen war eine Änderung der Schlafmittelgaben für ältere Patienten von PIM zu Nicht-PIM. Dementsprechend wurden die Medikamente unserer Wirkstoffliste in PIM und Nicht-PIM eingeteilt, um zu beurteilen, ob sich nicht nur die Zahl, sondern auch die Angemessenheit von Medikamentengaben in den zwei Zeiträumen verändert hat. Manche Medikamente gelten auf der PRISCUS-Liste erst ab einer bestimmten Dosierung als inadäquat für ältere Menschen. Bei diesen Medikamenten sollte zusätzlich erhoben werden, ob die Dosierung in diesem Sinne adäquat oder inadäquat war.

Wenn aus der Dokumentation nicht zu ersehen war, in welcher Dosierung ein Medikament verabreicht wurde, konnten wir durch die Lieferungslisten der Apotheke die tatsächliche Dosierung bestimmen.

\subsection{Durchführung der Datenerhebung}

Die Erhebung fand von Juni 2018 bis März 2019 statt - im Anschluss an eine Vorbereitungsphase seit Oktober 2017. Beteiligt waren zwei weitere Mitarbeiterinnen für deren Auswahl ein beruflicher medizinischer Hintergrund obligat war. Beide Mitarbeiterinnen erhielten eine standardisierte Anleitung für die Eingabe der Daten und wurden angehalten, den Richtlinien der SOP (s. Kapitel 4.6.2.2) zu folgen. Fragen konnten bei mir jederzeit oder in einer wöchentlichen Teambesprechung mit der Betreuerin Dr. Stephanie Heinemann, MPH besprochen werden.

\subsubsection{Vorbereitung der Datenerhebung}

Im Oktober 2017 haben wir ein Datenwörterbuch, eine Wirkstoffliste und eine Excel-Tabelle erstellt. Diese Instrumente wurden in einem Pretest mit 100 Patientenfällen unter drei Fragen überprüft (April 2018 bis Mai 2018): (1) ob alle zu erhebenden Daten in den Krankenakten auffindbar sind, (2) ob die Instrumente passen und (3) ob die erhobenen Daten auswertbar sind. Auf dieser Basis wurden einige Änderungen in den zu erhebenden Daten vereinbart und bis Juni 2018 eine Eingabemaske (siehe Kapitel 4.6.2.1) und eine SOP (s. Kapitel 4.6.2.2) entworfen, die eine hohe Verlässlichkeit, gerade auch bei Eingabe durch mehrere Personen gewährleisten sollten.

Die Daten aus dem Pretest wurden ausschließlich zur Verbesserung der Erhebungs-Instrumente genutzt und gingen nicht in die Haupterhebung ein. 


\subsubsection{Instrumente der Datenerhebung}

\subsubsection{Dateneingabemaske}

Um die Erhebung zu vereinheitlichen und Fehler zu vermeiden, wurde eine Dateneingabemaske durch einen externen Dienstleister programmiert, gesichert mit Benutzernamen und Passwort. Die Maske war unter einer SSL-gesicherten Internet-Adresse zu finden und konnte von den Computern des Krankenhaus Weende angesteuert werden.

Screenshots der einzelnen „Reiter“ dieser Eingabemaske, die Informationen der einzelnen Reiter und die in das jeweilige Feld einzutragenden Daten sind in der SOP (s. Anhang 8.4) zu finden.

\subsubsection{Standard Operating Procedure}

Um sicherzustellen, dass Informationen aus den Krankenakten zuverlässig und nachvollziehbar in die Dateneingabemaske übertragen werden, wurde eine SOP entworfen (s. Anhang 8.4). Da die Erhebung auf teilweise handschriftlich ausgefüllten Akten beruhte, gab es immer wieder neue Formulierungen und Arten der Aufzeichnung, die von der Krankenakte in die Dateneingabemaske übertragen werden mussten. Entscheidungen, wie etwas übertragen wurde, sollten festgehalten werden. So wurde die SOP im Lauf der Erhebung immer wieder erweitert. Das SOP-Kapitel „Fragestellungen, die aufkamen und wie wir sie gelöst haben“ ging auf spezielle Unklarheiten ein. Im Kapitel „Log der Ergänzungen“ wurde jede Ergänzung mit einem Datum festgehalten.

\subsubsection{Plausibilitätskontrollen}

Während der Erhebung und am Ende der Erhebung wurden regelmäßig Kontrollen der eingegebenen Daten durchgeführt, indem eine Excel-Tabelle des aktuellen Datensatzes heruntergeladen und durch die betreuenden Personen nach beispielsweise fehlenden Werten oder „unmöglichen“ Geburtsjahren durchsucht wurde.

\subsection{Endpunkte}

Für die primäre Forschungsfrage nach der Wirksamkeit der Intervention auf die Menge der verabreichten schlafanstoßenden Medikamente war primärer Endpunkt der Studie der Anteil der älteren Patienten, die ein schlafanstoßendes Medikament erhielten, im Vergleich von PräInterventionszeitraum und Post-Interventionszeitraum. Zweiter Endpunkt war die Häufigkeit der Gaben schlafanstoßender Medikamente, gemessen an Tagen mit Gaben dieser Medikamente bei älteren Patienten während ihres Krankenhausaufenthaltes. 
Anzahl älterer Patienten mit mind. 1 Hypnotikum

1. Primärer Endpunkt =

Anzahl aller älteren Patienten im Krankenhaus

Anzahl d. Tage mit Hypnotika-Gaben f. alle ält. Patienten

2. Primärer Endpunkt =

Anzahl der Behandlungstage aller älteren Patienten

Für die sekundäre Forschungsfrage nach der Wirksamkeit der Intervention auf die Angemessenheit der Verabreichungen von schlafanstoßenden Medikamenten an ältere Patienten war primärer Endpunkt der Anteilälterer Patienten, die mindestens einmal ein PIM zur Schlafförderung oder Beruhigung erhielten. Sekundärer Endpunkt war die Häufigkeit der Verabreichungen von PIM während des Krankenhausaufenthaltes. Dritter Endpunkt war die Anzahl der Verabreichungen der in der Leitlinie explizit empfohlenen Wirkstoffe (Melperon, Mirtazapin, Baldrian, Zolpidem $\leq 5 \mathrm{mg}$ ) im Vergleich zur Anzahl der Gaben aller schlafanstoßenden Medikamente.

Anzahl älterer Patienten mit mindestens 1 PIM

1. Sekundärer Endpunkt =

Anzahl aller älteren Patienten im Krankenhaus

Anzahl der Tage mit PIM-Gaben f. alle älteren Patienten

2. Sekundärer Endpunkt =

Anzahl der Behandlungstage aller älteren Patienten

Anzahl d. Gaben v. SOP-Medikamenten für alle ält. Pat.

3. Sekundärer Endpunkt =

Anzahl d. Gaben aller schlafanst. Med. f. alle ält. Patienten

\subsection{Stichprobenschätzung}

Für eine Schätzung des Stichprobenumfangs gingen wir, aufgrund einer Vorauswertung einer weiteren Dissertation (Neukirchen 2018) davon aus, dass 21\% der im Krankenhaus behandelten älteren Patienten mindestens einmal während ihres Aufenthalts ein oder mehrere Gaben aus Wirkstoffen der Benzodiazepin-Medikamentengruppe erhielten. Wünschenswertes Ziel der Intervention war die Senkung dieses Anteils um 5 Prozentpunkte, also von 21\% auf 
16\%. Um unter diesen Voraussetzungen einen Rückgang von 5 Prozent statistisch abzusichern, war eine Stichprobengröße von N = 982 in beiden Patientengruppen („Krankenakten" vor und nach Intervention) erforderlich (berechnet mittels G*Power 3.1M; (Faul et al. 2007)). Geplant war daher, 1000 Fälle (Krankenakten) je Zeitraum zu erheben.

\subsection{Kontrollkrankenhaus}

Eine der im Krankenhaus Weende entsprechenden Erhebung wurde im Krankenhaus St. Martini in Duderstadt durchgeführt. In diesem Haus fanden zwischen 2013 und 2017 keine Maßnahmen statt, die man vergleichbar dem Krankenhaus Göttingen-Weende als Intervention bezeichnen könnte, sodass dieses Krankenhaus als Kontrollkrankenhaus diente.

Die Erhebung im Krankenhaus St. Martini schloss im Jahr 2013 Fälle aus den Fachabteilungen Inneren Medizin und Allgemeinchirurgie ein. Im Jahr 2017 kamen zu diesen Abteilungen Fälle aus der zu dem Zeitpunkt neuen geriatrischen Station hinzu.

Für die Stichprobe galten dieselben Ein- und Ausschluss-Kriterien wie bei der Erhebung im Krankenhaus Weende (s. Kap. 4.4). Die SOP wurde vor der Erhebung in Duderstadt entsprechend angepasst und während der Erhebung, wie im Krankenhaus Weende, um wesentliche Entscheidungen ergänzt (s. a. Kap. 4.6.2.2). Die Datenerhebung erfolgte von November 2018 bis Juli 2019 und wurde zusammen mit einer weiteren, zuvor geschulten Mitarbeiterin durchgeführt.

Durch einen Vergleich mit der Kontrollgruppe sollte es gelingen, unbekannte Störfaktoren gewissermaßen indirekt zu erfassen bzw. zu kontrollieren - beispielsweise eine mögliche generelle Tendenz, weniger schlafanstoßende Medikamente zu verschreiben. Dieser Vergleich erlaubt es uns also, validere Rückschlüsse über den Zusammenhang zwischen der Intervention und der Verschreibung schlafanstoßender Medikamente zu ziehen.

\subsection{Datenauswertung}

Die Datenanalyse für die vorliegende Dissertation erfolgte mit SAS, Version 9.4. Die Daten wurden zunächst deskriptiv ausgewertet: Entweder nach absoluten und relativen Häufigkeiten oder nach Lageparametern (Mittelwert) und Streuungsmaßen (Standardabweichung). Ob sich der Anteil an Patienten, die Schlaf- oder Beruhigungsmittel im Krankenhaus erhielten, zwischen den beiden Zeiträumen signifikant veränderte, wurde mittels Fishers exakten Test überprüft. Als Signifikanzlevel wurde 5\% gewählt. Als Maß für die Effektgröße der Veränderung wurden Odds Ratios (ORs) und ihre 95\%-Konfidenzintervalle (KI) berechnet, d.h. das Verhältnis zwischen dem Anteil an Patienten mit Schlaf- oder Beruhigungsmittel nach und vor Intervention. Bei einer nach Fishers exaktem Test signifikanten Veränderung sollte auch das 95\%-KI für die ORs die „1“ nicht einschließen. Wenn sich die 95\%-KI beider Krankenhäuser nicht überlappen, kann darüber hinaus auch von einem signifikanten Unter- 
schied zwischen Kontroll- und Interventionskrankenhaus ausgegangen werden. Die statistische Absicherung beschränkte sich auf den primären Endpunkt bzw. den Vergleich zwischen den wichtigsten Substanzen.

\subsection{Genehmigung durch die Ethik-Kommission und Datenschutz}

Die Ethikkommission der Universitätsmedizin Göttingen hat das Studienprotokoll am 20.10.2017 genehmigt (Aktennummer 25/2/14, Amendement 5).

Alle an der unmittelbaren Datenerhebung Beteiligten haben sich durch Unterschreiben einer Datenschutzvereinbarung des Evangelischen Krankenhauses Weende zur strikten Verschwiegenheit verpflichtet. Eine entsprechende Datenschutzvereinbarung gab es auch im Kontrollkrankenhaus St. Martini Duderstadt. Die Dateneingabemaske war passwortgeschützt.

Die Daten wurden vorerst pseudonymisiert auf einem Server der Gesellschaft für wissenschaftliche Datenverarbeitung Göttingen (GWDG) gespeichert. Nach Beendigung der Datenerhebung wurde die für eine Pseudonymisierung notwendige Zuordnungsliste gelöscht und die Daten anonymisiert. 


\section{$5 \quad$ Ergebnisse}

\subsection{Das Krankenhaus}

Im Zeitraum vor der Intervention (2013) verfügte das Evangelische Krankenhauses Göttingen-Weende über 384 Betten und beschäftigte 130 Ärzte und 280 Pfleger (Störmer und Karaus 2013). Im zweiten Zeitraum 2017 waren es 433 Betten, 152 Ärzte und 371 Pfleger (Hampel und Karaus 2017). Die Beschäftigtenzahlen sind in Vollzeitkräften angegeben.

\subsection{Stichprobe}

Insgesamt wurden die Akten von 2156 Fällen (58\% Frauen) ausgewertet. Hier wird bewusst von „Fällen“ - statt Personen oder Patienten - gesprochen, weil vereinzelt Patienten bei mehreren Krankenhausaufenthalten auch mehrfach (als „Fälle“) ausgewertet werden; s.a. Kap 4.4. Ausgeschlossen wurden 72 Personen, die während des Krankenhausaufenthalts verstarben und 83 Fälle, die sich über die gesamte Dauer des Aufenthalts im Krankenhaus auf Intensivstation befanden*.

Die Stichprobe bestand somit aus 2009 Fällen, mindestens 65 Jahre alt. Die Stichproben waren in beiden Zeiträumen vergleichbar (s. Tabelle 2). Gemäß der in Kap. 5.1 dargestellten Entwicklung der Beschäftigten- und Bettenzahl ist es nicht erstaunlich, dass die zweite Stichprobe etwas größer $(\mathrm{N}=1049)$ als die erste in 2013 war $(\mathrm{N}=960)$.

Der Anteil der Patienten, die auf Stationen der chirurgischen Disziplinen behandelt wurden, war fast gleich dem Anteil, der auf Stationen der Inneren Medizin behandelt wurde. Deutlich weniger Patienten wurden auf den Stationen der Geriatrie behandelt. Zwei Drittel der Patienten wurden in beiden Zeiträumen aus der häuslichen Umgebung aufgenommen und ebenso viele hatten zum Zeitpunkt der Aufnahme keine Verordnung von schlafanstoßenden Medikamenten. Der Median des Aufenthalts auf der Intensivstation lag in beiden Zeiträumen bei 3 Tagen. Für die Berechnung der Tage mit Schlafmitteln wurden die Tage auf der Intensivstation oder Intermediate Care Station ausgeschlossen, da Medikamentengaben in diesen Zeiträumen nicht erfasst wurden (s. Kap. 4.4).

Die Anzahl der Patienten mit mindestens einer neurologisch-psychiatrischen Diagnose war auf Stationen der Geriatrie am höchsten, gefolgt von Stationen der Inneren Medizin und der Chirurgie. Dieses Muster ist in den Patientengruppen beider Zeiträume zu sehen (s. Tabelle 2). Eine detaillierte Übersicht über die Verteilung der Diagnosen nach Abteilung ist in Anhang 8.5 .

\footnotetext{
* Bei einigen ausgeschlossenen Fällen trafen beide Ausschlussgründe zu.
} 
Tabelle 2 Stichprobe

Prä-Intervention

\begin{tabular}{lcc} 
& $(\mathrm{N}=960)$ & $(\mathrm{N}=1049)$ \\
\hline Weibliches Geschlecht (\%) & 58,2 & 57,8 \\
Alter (Jahre); m (SD) & $79,4( \pm 7,5)$ & $80,0( \pm 7,7)$ \\
$\quad$ Frauen & $80,6( \pm 7,5)$ & $81,0( \pm 7,5)$ \\
$\quad$ Männer & $77,8( \pm 7,3)$ & $78,5( \pm 7,7)$
\end{tabular}

Station (\%)

Innere Medizin

39,9

37,1

Chirurgie

41,2

40,8

Geriatrie

22,1

Art der Aufnahme (\%)

Von Zuhause

66,3

67,9

Aus anderer Abteilung

12,6

12,6

Aus anderem Krankenhaus

6,2

5,3

Aus Pflegeheim

14,7

12,5

Unbekannt

0,3

1,7

\section{Verordnung schlafanstoßender Medikamente}

bei Aufnahme (\%)

Keine

67,1

68,3

Dauer

21,8

19,8

Bedarf

3,8

4,5

Dauer und Bedarf

3,5

3,2

Nicht erkennbar / vorhanden

3,9

4,2

Aufenthalt (Tage); m (SD)
Innere
$7,8( \pm 6,6)$
Chirurgie
$8,3( \pm 5,9)$
Geriatrie
$17,1( \pm 8,2)$
Intensivstation
$4,2( \pm 6,4)$
$9,7( \pm 7,6)$

$7,7( \pm 6,1)$

$8,1( \pm 8,2)$

$15,4( \pm 7,9)$

$5,0( \pm 9,4)$

$9,6( \pm 8,0)$

Alle Stationen

Patienten mit mindestens

einer neurol.-psychiatrischen Diagnose (\%); (n / N)
Innere Medizin
Chirurgie
Geriatrie

$24,5(94 / 383)$

$20,8(81 / 389)$

15,9 (63/395)

14,5 (62/428)

40,7 (74/182) 


\subsubsection{Neurologisch-psychiatrische Diagnosen}

Es wurde eine breite Auswahl von neurologisch-psychiatrischen Diagnosen erhoben, die im Zusammenhang mit Gaben schlafanstoßender Medikamente stehen könnten (s. Kap. 4.5.1). Auch die Verteilung der Diagnosen war in beiden Zeiträumen ähnlich (Tabelle 3). Die Mehrheit der Patienten, 75,9\% im Prä- und 77,7\% im Postinterventionszeitraum, hatte keine der Diagnosen. Der Anteil der Patienten, die mehr als eine der Diagnosen hatten, lag bei 4,3\% im ersten Zeitraum und 5,1\% im zweiten Zeitraum. Die häufigsten Diagnosen waren Demenz und Depression.

Tabelle 3 Neurologisch-psychiatrische Diagnosen*

\begin{tabular}{lccc}
\hline & $\begin{array}{c}\text { Prä-Intervention } \\
(\mathrm{N}=960)\end{array}$ & & $\begin{array}{c}\text { Post-Intervention } \\
(\mathrm{N}=1049)\end{array}$ \\
\cline { 2 - 3 } Diagnosen & $\mathbf{\%}$ & $\mathbf{\%}$ \\
\hline Patienten mit mind. 1 Diagnose & 24,1 & 22,3 \\
Demenz & 13,9 & 12,3 \\
Depression & 9,7 & 8,2 \\
Delir & 1,8 & 1,8 \\
Schlafmittelabhängigkeit & 1,4 & 0,9 \\
Angst oder Panik & 1,4 & 2,1 \\
Psychose & 0,5 & 1,4 \\
Schlafstörung & 0,6 & 0,6 \\
Andere** & 0 & 1,0 \\
\hline
\end{tabular}

*Mehrfachnennungen

**Andere neurologisch-psychiatrische Diagnosen, z. B. affektive Störung 


\subsection{Menge und Angemessenheit der verabreichten schlafanstoßenden Medikamente}

Der Anteil älterer Patienten, die während des Aufenthalts mindestens einmal ein schlafinduzierendes Medikament erhielten, lag im Zeitraum vor der Intervention bei 50,3\% (483/960). Dieser Anteil sank statistisch signifikant im Zeitraum nach der Intervention um 14 Prozentpunkte auf 36,3\% (381/1049; s. Tabelle 4). Die Signifikanz dieses Unterschiedes liegt bei p $<0,01$. Ausgedrückt in Odds Ratios lag der Effekt bei 0,56; 95\%-KI: 0,47-0,68. Eine Übersicht über Signifikanzwerte, Odds Ratios und Konfidenzintervalle ist in Anhang 8.6. Entsprechend gingen auch die Tage zurück, an denen Patienten mindestens ein schlafanstoßendes Medikament erhielten. Vor der Intervention wurden schlafanstoßende Medikamente an 37,3\% (3191/8556) der Krankenhaustage verabreicht. Nach der Intervention waren es nur noch 28,5\% (2689/9440) der Tage. Der Anteil der Patienten, der mindestens einmal ein potenziell inadäquates Medikament erhielt, verringerte sich von 21,5\% auf 8,7\%. Gleichzeitig sank auch die Häufigkeit ihrer Verabreichungen von $12,4 \%$ auf $5,5 \%$.

Tabelle 4 Zielerreichung der primären und sekundären Endpunkte Prä- und Postintervention

\begin{tabular}{|c|c|c|c|c|c|c|c|}
\hline & \multicolumn{3}{|c|}{ Prä-Intervention } & \multicolumn{3}{|c|}{ Post-Intervention } & \multirow{3}{*}{$\begin{array}{c}\text { Differenz } \\
\text { in } \\
\text { Prozent- } \\
\text { punkten }\end{array}$} \\
\hline & \multirow{2}{*}{$\begin{array}{c}\begin{array}{c}\text { Grund- } \\
\text { gesamtheit }\end{array} \\
\mathrm{N}\end{array}$} & \multicolumn{2}{|c|}{ Zielerreichung } & \multirow{2}{*}{$\begin{array}{c}\begin{array}{c}\text { Grund- } \\
\text { gesamtheit }\end{array} \\
\mathrm{N}\end{array}$} & \multicolumn{2}{|c|}{ Zielerreichung } & \\
\hline & & $\mathrm{n}$ & $(\%)$ & & $\mathrm{n}$ & $(\%)$ & \\
\hline \multicolumn{8}{|c|}{ Primäre Endpunkte } \\
\hline $\begin{array}{l}\text { Patienten mit } \\
\text { mindestens einem } \\
\text { schlafanstoßenden } \\
\text { Medikament }\end{array}$ & 960 & 483 & $(50,3)$ & 1049 & 381 & $(36,3)$ & $-14,0$ \\
\hline $\begin{array}{l}\text { Tage mit } \\
\text { mindestens einem } \\
\text { schlafanstoßenden } \\
\text { Medikament }\end{array}$ & 8556 & 3191 & $(37,3)$ & 9440 & 2689 & $(28,5)$ & $-8,8$ \\
\hline \multicolumn{8}{|c|}{ Sekundäre Endpunkte } \\
\hline $\begin{array}{l}\text { Patienten mit } \\
\text { mindestens einem } \\
\text { PIM }\end{array}$ & 960 & 206 & $(21,5)$ & 1049 & 91 & $(8,7)$ & $-12,8$ \\
\hline $\begin{array}{l}\text { Tage mit } \\
\text { mindestens einem } \\
\text { PIM }\end{array}$ & 8556 & 1058 & $(12,4)$ & 9440 & 515 & $(5,5)$ & $-6,9$ \\
\hline $\begin{array}{l}\text { Gaben } \\
\text { SOP-empfohlener } \\
\text { Medikamente }\end{array}$ & 4062 & 1919 & $(47,2)$ & 3435 & 2011 & $(58,5)$ & 11,3 \\
\hline
\end{tabular}


Bei Betrachtung des dritten sekundären Endpunkts sieht man, dass der Anteil der Gaben von in der SOP explizit empfohlenen Medikamenten (Melperon, Mirtazapin, Baldrian, Zolpidem $\leq 5 \mathrm{mg}$ ) von 47,2\% aller Medikamentengaben vor der Intervention auf 58,5\% nach der Intervention stieg.

Als nächstes sollte geprüft werden, inwieweit der Rückgang schlafanstoßender Medikamente auf allen Stationen zu beobachten war. Der größte Unterschied zeigte sich auf Stationen der chirurgischen Disziplinen, auf denen im Prä-Interventionszeitraum 50,1\% der älteren Patienten mindestens ein schlafanstoßendes Medikament erhielten, im Post-Interventionszeitraum 29,2\% der Patienten. Nicht nur die Zahl aller schlafanstoßenden Medikamente wurde weniger, sondern auch die Zahl potenziell inadäquater Medikamente, die auf chirurgischen Stationen von 31,6\% auf 10,3\% sank (s. Tabelle 5).

Tabelle 5 Schlafanstoßende Medikamente; nach Stationen

\begin{tabular}{|c|c|c|c|c|c|c|c|}
\hline & \multicolumn{3}{|c|}{ Prä-Intervention } & \multicolumn{3}{|c|}{ Post-Intervention } & \multirow{2}{*}{$\begin{array}{c}\text { Differenz } \\
\text { in } \\
\text { Prozent- } \\
\text { punkten }\end{array}$} \\
\hline & $\mathrm{N}$ & $\mathrm{n}$ & $(\%)$ & $\mathrm{N}$ & $\mathrm{n}$ & $(\%)$ & \\
\hline \multicolumn{8}{|c|}{ Patienten mit mindestens einem schlafanstoßenden Medikament } \\
\hline Innere Medizin & 383 & 169 & $(44,1)$ & 389 & 141 & $(36,2)$ & $-7,9$ \\
\hline Chirurgie & 395 & 198 & $(50,1)$ & 428 & 125 & $(29,2)$ & $-20,9$ \\
\hline Geriatrie & 182 & 116 & $(63,7)$ & 232 & 115 & $(49,6)$ & $-14,2$ \\
\hline \multicolumn{8}{|c|}{ Patienten mit mindestens einem PIM } \\
\hline Innere Medizin & 383 & 55 & $(14,4)$ & 389 & 25 & $(6,4)$ & $-7,9$ \\
\hline Chirurgie & 395 & 125 & $(31,6)$ & 428 & 44 & $(10,3)$ & $-21,4$ \\
\hline Geriatrie & 182 & 26 & $(14,3)$ & 232 & 22 & $(9,5)$ & $-4,8$ \\
\hline
\end{tabular}

N: Grundgesamtheit, n: Patienten mit mindestens einem schlafanstoßenden Medikament 


\subsection{Schlafanstoßende Medikamente für Patienten mit und ohne neurologisch-psychiatrische Diagnosen}

Tabelle 6 vergleicht die Gaben schlafanstoßender Medikamente für Patienten mit und ohne neurologisch-psychiatrische Diagnosen (s. Kap. 4.5.1). Der sich durch die bisherigen Ergebnisse ziehende Trend, zeigt sich auch beim Vergleich dieser beiden Gruppen: Sowohl Menge als auch Häufigkeit der verabreichten schlafanstoßenden Medikamente und potenziell inadäquater Medikamente waren nach der Intervention geringer als vor der Intervention.

Die Patienten mit Diagnosen ,starten“ in beinahe allen Endpunkten mit höheren Ausgangswerten. Vor der Intervention liegt - beispielsweise - der Anteil mit schlafanstoßenden Medikamenten in der Gruppe mit Diagnosen bei 67,1\% (155/231), in der Gruppe ohne Diagnosen bei 45,0\% (328/729).

Des Weiteren zu beobachten ist der meist größere Unterschied von Vorher zu Nachher in der Gruppe der Patienten ohne neurologisch-psychiatrische Diagnosen, verglichen mit der Gruppe mit diesen Diagnosen: Die Zahl der Patienten mit schlafanstoßenden Medikamenten ist um 16,2 Prozentpunkte geringer, wohingegen nur eine Differenz von 4,7 Prozentpunkten in der Gruppe von Patienten mit neurologisch-psychiatrischen Diagnosen zu sehen ist.

Dieser größere Unterschied ist auch in der Anzahl der Patienten mit potenziell inadäquaten Medikamenten zu sehen. Hier sieht man eine Differenz von 13,7 Prozentpunkten bei den Patienten ohne Diagnosen und eine Differenz von 9,7 Prozentpunkten bei den Patienten mit Diagnosen. Entsprechend geringer ist auch die Häufigkeit von Gaben potenziell inadäquater schlafanstoßender Medikamente im Vergleich beider Zeiträume, die durch die Anzahl der Tage mit diesen Medikamenten (,n“ in Tabelle 6) von allen im Krankenhaus verbrachten Tagen (ohne Intensivtage) („N“ in Tabelle 6) berechnet wird. Bei den Patienten ohne Diagnosen lag die Vorher-Nachher-Differenz bei 7,4 Prozentpunkten und bei den Patienten mit Diagnosen bei 5,7 Prozentpunkten.

Der Anteil von in der SOP empfohlenen Medikamenten stieg in der Gruppe der Patienten ohne Diagnosen an. Von 43,5\% im Zeitraum vor der Intervention zu 63,2\% im Zeitraum nach der Intervention. In der Gruppe der Patienten mit Diagnosen war der Anteil mit 52,6\% vor der Intervention höher und verblieb beinahe gleich mit 52,3\% nachher. 
Tabelle 6 Schlafanstoßende Medikamente für Patienten mit und ohne neurologischpsychiatrische Diagnosen*

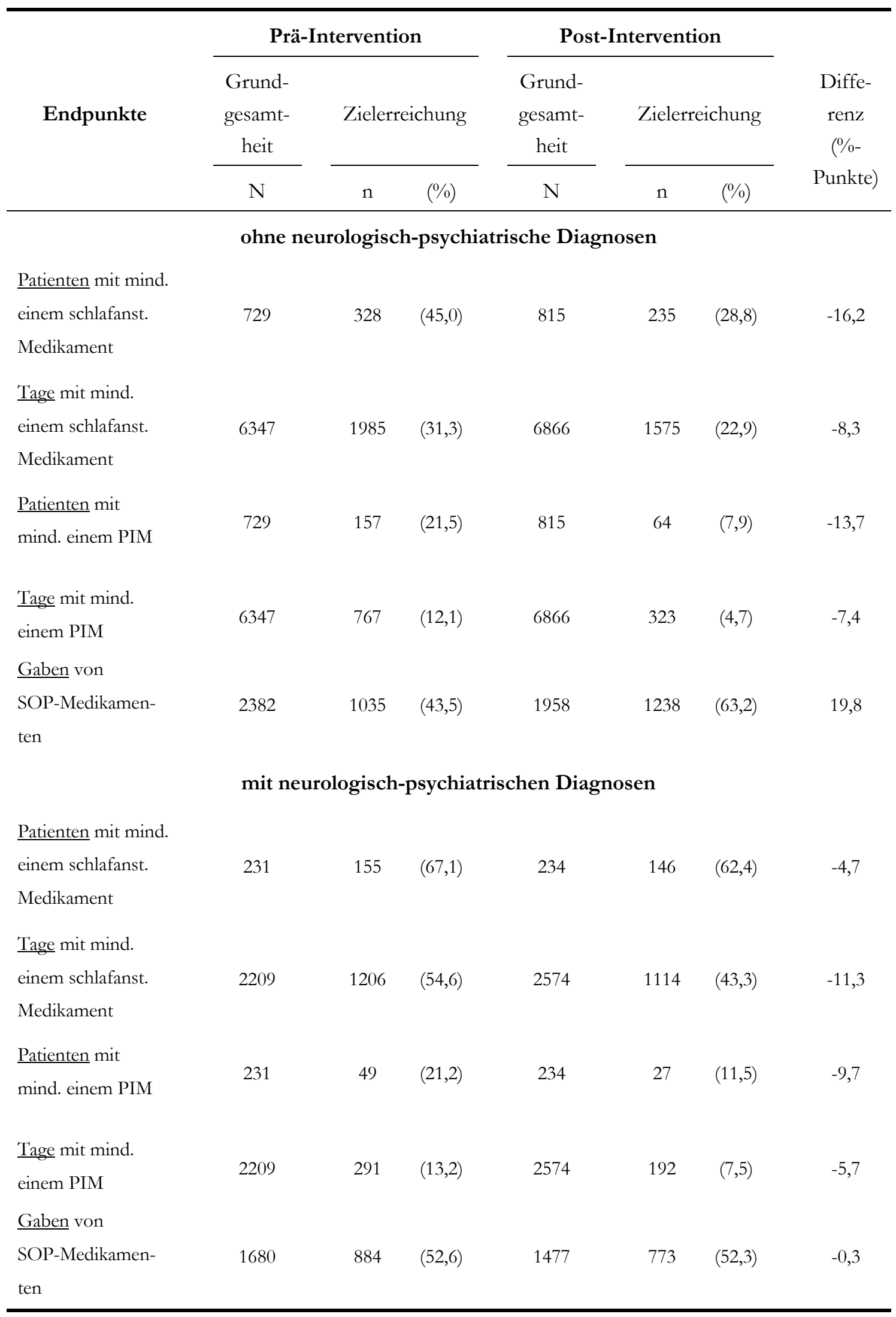

PIM: Potenziell inadäquates Medikament; SOP: Standard Operating Procedure 


\subsection{Häufigkeit der verschiedenen Wirkstoffe}

Betrachtet man die Patientenanteile mit schlafanstoßenden Medikamenten, aufgespaltet in verschiedene Wirkstoffgruppen, prägen sich die Unterschiede von vorher zu nachher verschieden stark aus (s. Abbildung 1, Tabelle 8). Während Wirkstoffe aus der Gruppe der Neuroleptika vor der Intervention bei fast der gleichen Anzahl der Patienten (14,1\%) verabreicht wurden, wie nach der Intervention (12,8\%) und bei Wirkstoffen aus der Z-Substanz-Gruppe ein statistisch signifikanter Unterschied von 4 Prozentpunkten $(p=0,03)$ zu sehen ist (OR $=0,62 ; 95 \%$-KI: 0,45-0,86; s. Anhang 8.6), fällt ein signifikanter Rückgang besonders stark in der Gruppe der Benzodiazepine auf, die vor der Intervention bei 24,3\% der Patienten verabreicht wurden, nach der Intervention bei 8,5\% ( $p<0,01)$. Die Effektstärke für diesen Rückgang lag in Odds Ratios ausgedrückt bei 0,31; 95\%-KI: 0,23-0,4. Den größten Anteil innerhalb der Benzodiazepine machte Lormetazepam aus, welches im Zeitraum vor der Intervention bei $13,2 \%$ der Patienten genutzt wurde $(11,7 \%$ in einer potenziell inadäquaten Dosierung), nach der Intervention bei 2,2\% (1,5\% in einer potenziell inadäquaten Dosierung).

Erkennbar ist auch, dass sich die Häufigkeit der Gaben von Lormetazepam vor und nach der Intervention unterschiedlich stark auf den Stationen ausprägte (Tabelle 7). Auf Stationen der chirurgischen Disziplinen, auf denen die Anzahl der Tage mit Lormetazepam vor und nach der Intervention am höchsten war, gab es auch den größten Sprung von vorher zu nachher: Lormetazepam wurde an 11,7\% der Tage des ersten Zeitraums verabreicht (10,2\% der Tage in potenziell inadäquater Dosierung). Im zweiten Zeitraum wurde es nur noch an $1,9 \%$ der Tage gegeben (1,3\% in potenziell inadäquater Dosierung).

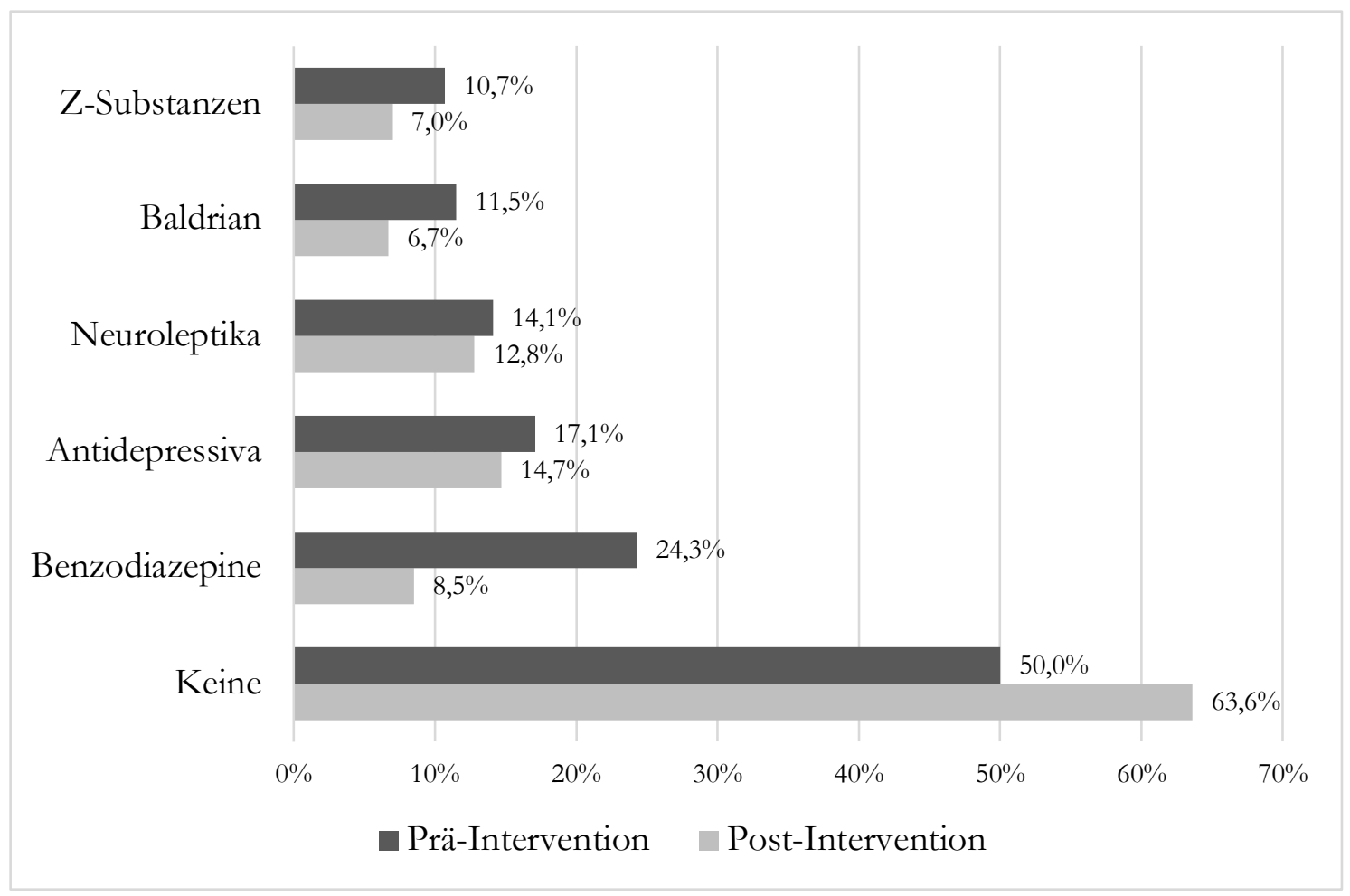

Abbildung 1 Patientenanteile mit schlafanstoßenden Medikamenten nach Wirkstoffgruppen. 
Tabelle 7 Tage mit Lormetazepam nach Abteilung und Zeitraum

\begin{tabular}{|c|c|c|c|c|c|c|c|c|c|c|}
\hline & \multicolumn{5}{|c|}{ Prä-Intervention } & \multicolumn{5}{|c|}{ Post-Intervention } \\
\hline & \multirow{2}{*}{$\begin{array}{c}\text { Tage } \\
\text { gesamt } \\
\text { N }\end{array}$} & \multicolumn{2}{|c|}{ Lormetazepam } & \multicolumn{2}{|c|}{ PID } & \multirow{2}{*}{$\begin{array}{l}\text { Tage } \\
\text { gesamt } \\
\text { N }\end{array}$} & \multicolumn{2}{|c|}{ Lormetazepam } & \multicolumn{2}{|c|}{ PID } \\
\hline & & $\mathrm{n}$ & $(\%)$ & $\mathrm{n}$ & $(\%)$ & & $\mathrm{n}$ & $(\%)$ & $\mathrm{n}$ & $(\%)$ \\
\hline \multicolumn{11}{|c|}{ Innere Medizin } \\
\hline & 2545 & 67 & $(2,6)$ & 57 & $(2,2)$ & 2785 & 2 & $(0,1)$ & 0 & $(0,0)$ \\
\hline \multicolumn{11}{|c|}{ Chirurgie } \\
\hline & 2947 & 346 & $(11,7)$ & 300 & $(10,2)$ & 3096 & 60 & $(1,9)$ & 39 & $(1,3)$ \\
\hline \multicolumn{11}{|c|}{ Geriatrie } \\
\hline & 3064 & 51 & $(1,7)$ & 49 & $(1,6)$ & 3559 & 22 & $(0,6)$ & 13 & $(0,4)$ \\
\hline
\end{tabular}

$\mathrm{N}$ : Krankenhaustage gesamt; $\mathrm{n}$ : Tage mit Lormetazepam; PID: Tage mit Lormetazepam in potenziell inadäquater Dosierung

Bei einem der Wirkstoffe, den wir aus der Z-Substanz-Wirkstoffgruppe erhoben haben, verringerte sich die Nutzung, während ein anderer vermehrt genutzt wurde: Zopiclon wurde weniger genutzt, die Anzahl der Patienten mit diesem Medikament lag im ersten Zeitraum bei 6,9\% (66/960). Im zweiten Zeitraum erhielten es nur noch 2,4\% (25/1049) der Patienten. Der Anteil der Patienten, die diesen Wirkstoff in einer potenziell inadäquaten Dosierung erhielt, ging ebenfalls zurück, von 3,8\% auf 1,7\%. Zolpidem wiederum wurde im zweiten Zeitraum bei einer größeren Zahl von Patienten verabreicht. Der Anteil stieg um 0,7 Prozentpunkte, von 3,9\% vor der Intervention zu 4,6\% nach der Intervention. Die Anzahl potenziell inadäquater Gaben von Zolpidem blieb währenddessen fast gleich.

Ein weiteres Medikament, dessen Verabreichungen sich im zweiten Zeitraum etwas erhöhten, ist Quetiapin aus der Neuroleptika-Gruppe. Vor der Intervention erhielten 2,8\% (27/960) der Patienten den Wirkstoff, nach der Intervention waren es 4,6\% (48/1049).

Betrachten wir alle schlafanstoßenden Medikamente ohne das pflanzliche Präparat Baldrian, das vor der Intervention 11,5\% der Patienten und nach der Intervention 6,7\% der Patienten erhielten, ist die Anzahl der Patienten, die schlafanstoßende Medikamente erhielten, weiterhin geringer im zweiten Zeitraum des Vorher-Nachher-Vergleichs, bewegt sich aber in beiden Zeiträumen bei rund 4 Prozentpunkten weniger mit 45,8\% vor und 32,7\% nach der Intervention. 
Tabelle 8 Patientenanteile mit schlafanstoßenden Medikamenten* nach Wirkstoffgruppen und potenziell inadäquater Dosierung**

\begin{tabular}{|c|c|c|c|c|c|c|}
\hline & \multicolumn{3}{|c|}{$\begin{array}{l}\text { Prä-Intervention } \\
\text { (n = } 960 \text { Patienten) }\end{array}$} & \multicolumn{3}{|c|}{$\begin{array}{l}\text { Post-Intervention } \\
(\mathrm{n}=1049 \text { Patienten })\end{array}$} \\
\hline & $\mathrm{n}$ & $\%$ & PID $(\%)$ & $\mathrm{n}$ & $\%$ & PID $(\%)$ \\
\hline Keine schlafanst. Medik. & 477 & 49,7 & & 667 & 63,6 & \\
\hline Alle schlafanst. Medik. & 483 & 50,3 & & 381 & 36,3 & \\
\hline Ohne Baldrian & 440 & 45,8 & & 343 & 32,7 & \\
\hline Benzodiazepine & 233 & 24,3 & & 89 & 8,5 & \\
\hline Lormetazepam & 127 & 13,2 & $>0,5 \mathrm{mg}(11,7)$ & 23 & 2,2 & $>0,5 \mathrm{mg}(1,5)$ \\
\hline Lorazepam & 55 & 5,7 & $>2 \mathrm{mg}(0,2)$ & 46 & 4,4 & $>2 \mathrm{mg}(0,4)$ \\
\hline Oxazepam & 18 & 1,9 & $>60 \mathrm{mg}(0,1)$ & 6 & 0,6 & $>60 \mathrm{mg}(0)$ \\
\hline Diazepam & 14 & 1,5 & J.G. $(1,5)$ & 2 & 0,2 & J.G. $(0,2)$ \\
\hline Temazepam & 7 & 0,7 & J.G. $(0,7)$ & 3 & 0,3 & J.G. $(0,3)$ \\
\hline Andere Benzodiazepine & 12 & 1,3 & & 9 & 0,9 & \\
\hline Z-Substanzen & 103 & 10,7 & & 73 & 7,0 & \\
\hline Zopiclon & 66 & 6,9 & $>7,5 \mathrm{mg}(3,8)$ & 25 & 2,4 & $>7,5 \mathrm{mg}(1,7)$ \\
\hline Zolpidem & 37 & 3,9 & $>5 \mathrm{mg}(2,7)$ & 48 & 4,6 & $>5 \mathrm{mg}(2,6)$ \\
\hline Neuroleptika & 135 & 14,1 & & 134 & 12,8 & \\
\hline Melperon & 53 & 5,5 & n.a. & 50 & 4,8 & n.a. \\
\hline Quetiapin & 27 & 2,8 & n.a. & 48 & 4,6 & n.a. \\
\hline Prothipendyl & 19 & 2,0 & n.a. & 11 & 1,0 & n.a. \\
\hline Promethazin & 14 & 1,5 & n.a. & 1 & 0,1 & n.a. \\
\hline Haloperidol & 8 & 0,8 & $>2 \mathrm{mg}(0,2)$ & 3 & 0,3 & $>2 \mathrm{mg}(0,1)$ \\
\hline Risperidon & 7 & 0,7 & n.a. & 12 & 1,1 & n.a. \\
\hline Andere Neuroleptika & 7 & 0,7 & & 9 & 0,9 & \\
\hline Antidepressiva & 164 & 17,1 & & 154 & 14,7 & \\
\hline Mirtazapin & 130 & 13,5 & n.a. & 137 & 13,1 & n.a. \\
\hline Amitriptylin & 11 & 1,1 & J.G. $(1,1)$ & 9 & 0,9 & J.G. $(0,9)$ \\
\hline Doxepin & 8 & 0,8 & J.G. $(0,8)$ & 4 & 0,4 & J.G. $(0,4)$ \\
\hline andere Antidepressiva & 12 & 1,3 & & 4 & 0,4 & \\
\hline Andere & 111 & 11,6 & & 71 & 6,8 & \\
\hline Baldrian & 110 & 11,5 & n.a. & 70 & 6,7 & n.a. \\
\hline
\end{tabular}

* Die Tabelle wurde gekürzt auf Wirkstoffe, die in einem der Zeiträume mindestens 5 Patienten verabreicht wurden. Eine ungekürzte Tabelle ist in Anhang 8.7.

** PID: Potenziell inadäquate Dosierung; J.G.: Jede Gabe; n.a.: nicht anwendbar 


\subsection{Kontrollkrankenhaus Duderstadt}

\subsubsection{Stichprobe}

Das Krankenhaus St. Martini in Duderstadt verfügte im ersten Erhebungszeitraum (2013) über 126 Betten und beschäftigte 33 Ärzte und 88 Pflegerinnen und Pfleger (Hackenfort und Kohlstedde 2013). Im zweiten Zeitraum (2017) waren es 140 Betten, 44 Ärzte und 105 Pflegerinnen und Pfleger (Bartels und Kohlstedde 2017). Die Beschäftigtenzahlen sind in Vollzeitkräften angegeben. In Duderstadt wurden die Akten von 817 Fällen ausgewertet. Davon wurden 35 Fälle ausgeschlossen, da die Patienten während des Aufenthalts verstarben, 6 Patienten, die nur auf der Intensivstation waren und 1 Patient, der keine Nacht im Krankenhaus verbrachte. Die Stichprobe in Duderstadt bestand somit aus 777 Fällen. Sie war, entsprechend der Entwicklung der Beschäftigten- und Bettenzahl, im ersten Zeitraum etwas kleiner $(\mathrm{N}=366)$ als im zweiten $(\mathrm{N}=411)$.

In beiden Zeiträumen wurden die meisten Patienten auf Stationen der Inneren Medizin behandelt (Tabelle 9). Den zweitgrößten Anteil machten Patienten auf chirurgischen Stationen aus. Im zweiten Zeitraum kamen Patienten der geriatrischen Abteilung dazu, die den kleinsten Anteil ausmachten. Der Median des Aufenthalts auf der Intensivstation lag in beiden Zeiträumen bei 2 Tagen.

Die Verteilung der Patienten, die neurologisch-psychiatrische Diagnosen hatten war in beiden Zeiträumen ähnlich (Tabelle 10). Die Mehrheit der Patienten, 74,0\% im ersten und $72,7 \%$ im zweiten Zeitraum, hatte keine der neurologisch-psychiatrischen Diagnosen. Der Anteil, der mehr als eine der Diagnosen hatte, lag bei 4,9\% im ersten Zeitraum und 3,2\% im zweiten. Die häufigsten Diagnosen waren Demenz und Depression. 
Tabelle 9 Stichprobe des Kontrollkrankenhauses

2013

2017

\begin{tabular}{lcc} 
& $(\mathrm{N}=366)$ & $(\mathrm{N}=411)$ \\
\hline Weibliches Geschlecht (\%) & 59,0 & 55,5 \\
Alter (Jahre); m (SD) & $79,3( \pm 6,9)$ & $80,0( \pm 7,3)$ \\
$\quad$ Frauen & $80,6( \pm 6,8)$ & $80,7( \pm 7,4)$ \\
$\quad$ Männer & $77,7( \pm 6,8)$ & $78,4( \pm 6,9)$
\end{tabular}

Station $(\%)$

$\begin{array}{lcc}\text { Innere Medizin } & 67,5 & 62,0 \\ \text { Chirurgie } & 32,5 & 28,7 \\ \text { Geriatrie } & 0 & 9,3\end{array}$

Art der Aufnahme (\%)

$\begin{array}{lcc}\text { Von Zuhause } & 79,8 & 74,5 \\ \text { Aus anderer Abteilung } & 1,6 & 9,0 \\ \text { Aus anderem Krankenhaus } & 0,3 & 1,7 \\ \text { Aus Pflegeheim } & 18,0 & 13,6 \\ \text { Unbekannt } & 0,3 & 1,2\end{array}$

Verordnung schlafanstoßender Medikamente

bei Aufnahme (\%)

Keine

64,0

65,5

Dauer

24,6

19,7

Bedarf

4,9

5,8

Dauer und Bedarf

1,4

5,4

Nicht erkennbar / vorhanden

5,2

3,7

Aufenthalt (Tage); m (SD)
Innere
$6,7( \pm 4,9)$
$7,2( \pm 5,1)$
Chirurgie
$9,4( \pm 7,0)$
$7,7( \pm 6,0)$
Geriatrie
0
$17,8( \pm 3,2)$
Intensivstation
$4,0( \pm 6,7)$
$3,8( \pm 4,4)$
$7,6( \pm 5,8)$
$8,4( \pm 6,0)$

Alle Stationen

\section{Patienten mit mindestens}

einer neurologisch-psychiatrischen Diagnose (\%); (n / N)
Innere Medizin
$26,3(65 / 247)$
$27,8(68 / 255)$
Chirurgie
$25,2(30 / 119)$
$20,3(23 / 118)$
Geriatrie
0
$44,7(16 / 38)$ 
Tabelle 10 Patienten mit neurologisch-psychiatrischen Diagnosen im Kontrollkrankenhaus

\begin{tabular}{|c|c|c|}
\hline & $\begin{array}{c}2013 \\
(N=366)\end{array}$ & $\begin{array}{c}2017 \\
(\mathrm{~N}=411)\end{array}$ \\
\hline Diagnosen* & $\%$ & $\%$ \\
\hline Patienten mit mind. 1 Diagnose & 26,0 & 27,3 \\
\hline Demenz & 13,9 & 15,6 \\
\hline Depression & 11,5 & 10,7 \\
\hline Delir & 1,6 & 0,7 \\
\hline Schlafmittelabhängigkeit & 0,0 & 0,0 \\
\hline Angst oder Panik & 1,6 & 2,2 \\
\hline Psychose & 1,6 & 0,7 \\
\hline Schlafstörung & 0,8 & 0,5 \\
\hline Andere** & 0,0 & 0,2 \\
\hline
\end{tabular}

*Mehrfachnennungen

**Andere neurologisch-psychiatrische Diagnosen, z. B. affektive Störung

\subsubsection{Menge der verabreichten schlafanstoßenden Medikamente im Kontrollkrankenhaus}

Im Kontrollkrankenhaus lag der Anteil der Patienten, die während des Aufenthalts mindestens einmal ein schlafanstoßendes Medikament erhielten bei 43,2\% (158/366) im ersten Zeitraum und bei 36,5\% (150/411) im zweiten Zeitraum (Tabelle 11). Die Reduktion war mit einem P-Wert von 0,66 statistisch nicht signifikant (s.a. Anhang 8.6). Im Interventionskrankenhaus war die Reduktion stärker als im Kontrollkrankenhaus, wie sich im Vergleich der Odds Ratios als Effektgrößen zeigt (0,56 vs. 0,76), allerdings nicht statistisch signifikant, da sich die Konfidenzintervalle der ORs überlappen.

Von den Patienten ohne neurologisch-psychiatrische Diagnosen erhielten im ersten Zeitraum 35,4\% schlafanstoßende Medikamente, im zweiten Zeitraum 27,4\%. Bei den Patienten mit diesen Diagnosen war der Anteil mit schlafanstoßenden Medikamenten höher, mit 65,3\% im ersten Zeitraum und 60,7\% im zweiten Zeitraum. 
Tabelle 11 Patienten mit mindestens einem schlafanstoßenden Medikament im Kontrollkrankenhaus, mit und ohne neurologisch-psychiatrische Diagnosen

\begin{tabular}{|c|c|c|c|c|c|c|c|}
\hline & \multicolumn{3}{|c|}{2013} & \multicolumn{3}{|c|}{2017} & \multirow{2}{*}{$\begin{array}{c}\text { Differenz } \\
\text { in } \\
\text { Prozent- } \\
\text { punkten }\end{array}$} \\
\hline & $\mathrm{N}$ & $\mathrm{n}$ & $(\%)$ & $\mathrm{N}$ & $\mathrm{n}$ & $(\%)$ & \\
\hline \multicolumn{8}{|l|}{ Alle Patienten } \\
\hline & 366 & 158 & $(43,2)$ & 411 & 150 & $(36,5)$ & $-6,9$ \\
\hline \multicolumn{8}{|c|}{$\begin{array}{l}\text { Patienten ohne } \\
\text { neurologisch-psychiatrische Diagnosen }\end{array}$} \\
\hline & 271 & 96 & $(35,4)$ & 299 & 82 & $(27,4)$ & $-8,0$ \\
\hline \multicolumn{8}{|c|}{$\begin{array}{l}\text { Patienten } \underline{\text { mit }} \\
\text { neurologisch-psychiatrischen Diagnosen }\end{array}$} \\
\hline & 95 & 62 & $(65,3)$ & 112 & 68 & $(60,7)$ & $-4,6$ \\
\hline
\end{tabular}

$\mathrm{N}$ : Grundgesamtheit, n: Patienten mit mindestens einem schlafanstoßenden Medikament 


\section{Diskussion}

Nach einer Intervention ging der Anteil von Patienten, die ein schlafanstoßendes Medikament erhielten, signifikant um knapp 15 Prozentpunkte zurück (von 50,3\% auf 36,3\%). Entsprechend verringerte sich auch der Anteil der Tage im Krankenhaus, an denen Patienten solche Medikamente erhielten (von über 37\% auf 29\%). Ebenso sank der Anteil der Patienten mit potenziell inadäquater Medikation von $22 \%$ auf $9 \%$. Am stärksten waren diese Änderungen auf Stationen der Chirurgie. Insbesondere der Anteil von Patienten mit Benzodiazepinen verringerte sich signifikant von $24 \%$ auf $8 \%$.

\subsection{Stärken und Schwächen der Untersuchung}

\subsubsection{Chart Review}

Für einen retrospektiven Vergleich der Menge verabreichter schlafanstoßender Medikamente in zwei Zeiträumen ist die Auswertung von Patientenakten gut geeignet. Nicht nur konnten wir direkt nach Planungs- und Pilotphasen mit der Erhebung beginnen. Die Akten waren zu jeder Zeit zugänglich und die Datenerhebung fand in einer ruhigen Arbeitsumgebung statt. Die Informationen, die für die Endpunkte der Studie erfasst werden sollten, konnten objektiv erfasst werden.

Ein Nachteil der Arbeit mit Patientenakten ist die eingeschränkte Verlässlichkeit der Informationen. Man weiß im Regelfall nicht, wie und von wem Patienteninformationen in die Akten eingetragen wurden und welcher Informationsverlust dabei entstand (Panacek 2007). Die Aufnahmemedikation war in unserer Erhebung bei jeweils rund 4\% der Patienten in beiden Zeiträumen nicht herauszufinden oder nicht dokumentiert. Und selbst wenn die Aufnahmemedikation dokumentiert war, kann die Fehlerquote hoch sein (Beers et al. 1990; Caglar et al. 2011). Die Genauigkeit der Dokumentation und die Schlüssigkeit der Patientendaten korrelieren negativ mit der Anzahl der an der Dokumentation beteiligten Ärzte, Pfleger, Therapeuten und Auszubildenden. Gleichzeitig hat die stationäre Medikation eine zentrale Rolle in der Krankenhausbehandlung so dass von einer hohen Sorgfalt der Dokumentation auszugehen ist und auch mögliche Fehleinträge im weiteren Verlauf korrigiert werden dürften.

Als Richtlinie für die Durchführung einer Aktenauswertung listet Panacek „10 Gebote“ (s. Tabelle 1), die - bis auf eine Ausnahme - ganz oder weitgehend beachtet wurden: Forschungsfragen und Auswahlkriterien waren vor Beginn der Durchführung festgelegt; Variablen und Richtlinien wurden vor der Erhebung festgelegt (in einer SOP; s. a. 4.6.2.2); die Erhebenden („Datenabstraktoren“) hatten einen medizinischen Hintergrund, wurden vor der Erhebung eingearbeitet und in der Anfangsphase eng betreut; durch die Arbeit mit einer Dateneingabemaske und der SOP war eine Kontinuität in der Erhebung sichergestellt; die Datenbank wurde regelmäßig durch zwei Personen auf Plausibilität und Doppelt-Einträge 
überprüft und gegebenenfalls korrigiert; es fanden regelmäßige Treffen zwischen den Datenabstraktoren und Studienleitern statt.

Die Qualitätssicherung der Datenerhebung (Interrater-Reliabilität) haben wir anhand einer kleinen Akten-Stichprobe überprüft. Hier haben wir zwar keinen statistischen Wert ermittelt, können aber einigermaßen gut Übereinstimmung und Abweichung überprüfen. Die empfohlene „Verblindung“ der Datenabstraktoren war logischerweise nicht möglich. In Anbetracht der doch engen Zusammenarbeit aller Beteiligten wäre es auch sehr „künstlich“ gewesen, zwei an der Datenerhebung Beteiligten nicht über das Ziel der Studie aufzuklären.

\subsubsection{Stichprobe}

Die sehr ähnlichen Basischarakteristika der beiden Stichproben sprechen dafür, dass sie miteinander vergleichbar sind. Die Stichproben wurden aus jeweils zwei gleichen Zeiträumen erhoben, sodass saisonale Einflüsse auszuschließen sind. Um sicherzustellen, dass wir klinisch relevante Unterschiede absichern können, fand vor der Erhebung eine Power-Berechnung statt.

Die in Kapitel 4.4 beschriebene Gegebenheit, dass für die Erhebung „Fälle“ anstatt „Patienten" bearbeitet wurden und derselbe Patient mehrfach in der Studie auftauchen kann führt dazu, dass die Patientenzahl der Studie höher ist als die tatsächliche Zahl von Patienten aus dem Zeitraum. Da es mehrere chirurgische, internistische und geriatrische Abteilungen gibt und das Verfahren, bei einer Verlegung - auch innerhalb einer Disziplin - einen neuen Fall zu erstellen konsequent durchgezogen wurde, entspricht die Verteilung der Patienten auf den Stationen nicht vollkommen der tatsächlichen. Ein Stationswechsel, der häufiger vorkam, war der Wechsel von der akuten zur rehabilitativen geriatrischen Station. Die Zahl der geriatrischen Fälle überschätzt dementsprechend die tatsächliche Zahl. Da allerdings in beiden Stichproben so verfahren wurde, wird die interne Vergleichbarkeit dadurch nicht beeinträchtigt.

Die Stichprobe aus der Studie von Arnold et al. (2017), die ebenfalls im Rahmen des Schlafmittelprojekts stattfand, ist aufgrund ähnlicher Ein- und Ausschlusskriterien vergleichbar mit der Stichprobe dieser Studie. Das durchschnittliche Alter und die Geschlechterverteilung fallen sehr ähnlich aus. Unterschiede in einigen Charakteristika sind wahrscheinlich durch das im vorherigen Absatz erläuterte Verfahren der Betrachtung eines Patienten als einen neuen Fall bei Neuaufnahme oder Verlegung zu erklären. Auf diese Weise verfuhren auch Arnold et al. (2017, S. 19), allerdings nicht bei Verlegungen innerhalb der Geriatrie. Punkte, die mutmaßlich durch dieses Verfahren in unserer Studie anders ausfallen, sind der größere Anteil von Patienten auf geriatrischen Stationen, der hohe Anteil von Patienten, die aus einer anderen Abteilung aufgenommen wurden und der kürzere durchschnittliche Aufenthalt auf Stationen der Geriatrie.

Der Anteil der Patienten mit Demenz ist in unserer Studie mit 13,9\% bzw. 12,3\% in beiden Untersuchungszeiträumen etwas höher als der Anteil in der erwähnten Arbeit von Arnold et 
al. (2017) (10,8\%). Ursache für die kleine Differenz könnte die Methode der Erhebung sein: In der Erhebung von Arnold et al. (2017) wurden Haupt- und Nebendiagnosen des Entlassungsbriefs nach Textbausteinen durchsucht, die für eine Demenz-Diagnose sprachen. In der vorliegenden Arbeit wurden sämtliche Arztbriefe des Patienten nach Haupt- und Nebendiagnosen durchsucht.

Unter Berücksichtigung der methodischen Unterschiede ist davon auszugehen, dass die Stichproben vergleichbar sind mit der von Arnold et al. (2017).

Im Vergleich mit dem Durchschnitt anderer deutscher Krankenhäuser ist der Anteil der Frauen in unserer Stichprobe etwas höher. Von den Patienten, die 65 Jahre und älter waren, machten Frauen im Jahr 2013 53,8\% aus, 2017 52,8\% (Statistisches Bundesamt 2013; Statistisches Bundesamt 2017). In unserer Stichprobe war dieser Anteil weiblicher Patienten um jeweils rund 5 Prozentpunkte höher, mit 58,2\% im ersten und 57,8\% im zweiten Zeitraum. Eine Erklärung für den Unterschied in der Geschlechterverteilung könnte sein, dass das Krankenhaus Weende nicht über gynäkologische Abteilungen verfügt.

\subsubsection{Medikamentenauswahl}

Die Definition von „Hypnotika“ ist sehr klar. Nach Steiger et al. (2019) sind Hypnotika schlaffördernde Arzneimittel. Welche Medikamente in Studien dann aber tatsächlich als Hypnotika bezeichnet und erforscht werden, ist schon nicht mehr so klar. Anhang 8.3 zeigt eine Auswahl von Medikamenten, die in verschiedenen Studien als Hypnotika definiert wurden. Die Definition von schlafanstoßenden Medikamenten in unserer Studie bezog sich primär auf deren sedierende Eigenschaft (s. Anhang 8.2). Wir wollten mit der Auswahl unserer Wirkstoffe einen möglichst großen Umfang an schlafanstoßenden Medikamenten decken, sodass beispielsweise auch Baldrian zu den sedierenden Wirkstoffen gezählt wird. Strategie der Zusammenstellung der Wirkstoffliste und die Wirkstoffe wurden in einem Gespräch mit zwei klinisch tätigen Ärzten reflektiert. Weiterer Inhalt des Gesprächs war eine Reflektion der Diagnosen (s. Kap. 6.1.4). Die Auswahl der Wirkstoffe für diese Arbeit erfolgte sehr gründlich und bedacht und stellt eine Stärke dar.

\subsubsection{Diagnosen}

Mit einer breiten Auswahl von neurologisch-psychiatrischen Diagnosen (s. Kapitel 4.5.1) wollten wir überprüfen, ob ein möglicher Rückgang von schlafanstoßenden Medikamenten auch die „richtigen“ Patienten (ohne solche Diagnosen) getroffen hat, da die untersuchten Medikamente in einigen Fällen auch andere Indikationen haben. Benzodiazepine beispielsweise werden in akuten Angstzuständen verabreicht, sedierende Neuroleptika bei Psychosen und sedierende Antidepressiva bei affektiven Störungen. Die Indikation eines Medikaments ist allerdings nicht immer aus der Krankenakte zu ersehen. Bei der Auswahl dieser Diagnosen orientierten wir uns vor allem an den häufigsten psychischen Krankheitsbildern wie Demenz 
und Depression und dazu noch anderen relevanten Diagnosen (Saß et al. 2009, S. 31). Trotzdem erhoben wir nicht alle möglichen Diagnosen. Ein Beispiel für eine Diagnose, die wir nicht erhoben haben, ist „Zustand nach Schlaganfall“: Kim et al. (2002) fanden in knapp einem Drittel der Patienten, die 3 bis 12 Monate nach Schlaganfall befragt wurden, die Unfähigkeit, Wut und Aggression zu kontrollieren. Aggressionszustände dieser Art könnten eine Indikation für Gaben schlafanstoßender Medikamente sein. Bei Unklarheiten über Diagnosen überlegten wir uns jeweils Maßnahmen, um diese zu beheben. Ein Beispiel für eine solche Maßnahme waren Gespräche mit denjenigen, die diese Diagnosen vergeben oder die ,große Krankenhauspolitik“ bestimmen (s.a. Kapitel 6.1.3).

Ferner ist auf die Genauigkeit der Dokumentation von Diagnosen einzugehen: Bei der Erhebung fiel ein deutlicher Unterschied in der Qualität der Dokumentation der Diagnosen auf. In Arztbriefen der Geriatrischen Rehabilitation war die Auflistung von Diagnosen sehr umfassend erhoben, während sich die Diagnosen in den Arztbriefen der unfallchirurgischen Stationen oftmals vor allem auf das aktuelle Problem bezogen. Die Differenzen können zur Folge haben, dass die Anzahl von Patienten mit neurologisch-psychiatrischen Diagnosen in unserer Studie unterschätzt wird. Da die Patientenanteile der verschiedenen Stationen vor und nach der Intervention allerdings mehr oder weniger identisch waren schränken die möglichen Unterschiede in den Diagnosen die Qualität der Ergebnisse dieser Studie nur bedingt ein.

\subsubsection{Studiendesign}

Das Quasi-Experiment ist eine Methode, um einen kausalen Einfluss einer unabhängigen Variable (Intervention) auf abhängige Variablen (Menge und Angemessenheit der schlafanstoßenden Medikamente, Patientencharakteristika) zu überprüfen. In unserem Quasi-Experiment untersuchten wir den kausalen Einfluss der Intervention (unabhängige Variable) auf die Menge und Angemessenheit schlafanstoßender Medikamente und Basischarakteristika der Patienten (abhängige Variablen). Im Unterschied zum Experiment erfolgt beim QuasiExperiment keine Randomisierung der Versuchspersonen. Stattdessen wird mit den vorgefundenen Gruppen gearbeitet - in unserer Studie sind das die Patienten aus den zwei Zeiträumen. Dies kann dazu führen, dass sich die Vergleichsgruppen anhand unkontrollierter Merkmale systematisch unterscheiden. Veränderungen des Effektes sind nicht eindeutig auf die unabhängige Variable zurückzuführen, weil sie auch durch die Störvariablen bedingt sein können. Die interne Validität von quasi-experimentellen Untersuchungen ist daher geringer als die von experimentellen Untersuchungen (Döring und Bortz 2016, S. 199). Dementsprechend kann eine eindeutige Kausalbeziehung zwischen unabhängiger Variable und abhängiger Variable nicht abgeleitet werden (Wirtz 2020). Wir versuchten diese unkontrollierten Merkmale durch einen Vergleich in einem weiteren Krankenhaus zu überprüfen. 
Da wir Patienten in nur einem Krankenhaus untersuchten, sind unsere Beobachtungen hierarchisch dem Krankenhaus zugeordnet und wir ,überschätzen“ dementsprechend die Stichprobe. Ein optimales Design für die Untersuchung der Forschungsfragen wäre eine clusterrandomisierte Studie gewesen, in der Stationen bzw. Krankenhäuser zufällig einer Interventionsgruppe bzw. einer Kontrollgruppe zugeordnet werden. Eine Überprüfung der Intervention in einem entsprechend erweiterten Ansatz wäre wünschenswert.

\subsubsection{Kontrollkrankenhaus}

Durch die unterschiedliche Lage der Krankenhäuser bestehen sehr wahrscheinlich Differenzen in der Sozialstruktur des Einzugsbereichs, der örtlichen Arztdichte und anderen Punkten. In der Versorgungsstufe als Häuser der Grund- und Regelversorgung entsprechen sich die Krankenhäuser. Das Kontrollkrankenhaus verfügte ebenfalls über internistische und chirurgische Stationen, im zweiten Zeitraum darüber hinaus auch über eine geriatrische Abteilung. Das Kontrollkrankenhaus war etwas kleiner als das Interventionskrankenhaus. Durch die Ergänzung des Kontrollkrankenhauses mit einer geriatrischen Abteilung im zweiten Zeitraum entsprachen sich die Fachabteilungen in beiden Krankenhäusern, was den Vergleich leichter gestaltet.

Die Stichprobe ist - der Größe des Kontrollkrankenhauses entsprechend - etwas kleiner als im Weender Krankenhaus. Wir finden auch in dieser Stichprobe einen Anstieg in der Patientenzahl vom ersten zum zweiten Erhebungszeitraum, womöglich durch das Hinzukommen der geriatrischen Abteilung. Der geringere Anteil der Patienten auf Stationen der Chirurgie (im Vergleich zum Interventionskrankenhaus) hängt womöglich mit der kleineren Anzahl an chirurgischen Fachabteilungen zusammen. Beim Betrachten der Verteilung der Patienten auf die Stationen, der Aufnahmeart, der Aufenthaltsdauer und der Anteile der Patienten mit neurologisch-psychiatrischen Diagnosen auf den jeweiligen Stationen fallen Unterschiede auf, die sich mit dem Vorhandensein einer geriatrischen Abteilung im zweiten Zeitraum erklären lassen. Bei der Betrachtung der Häufigkeiten verschiedener neurologisch-psychiatrischer Diagnosen (wie etwa Demenz und Depression) ist festzustellen, dass die Stichproben sowohl innerhalb des Krankenhauses in beiden Zeiträumen, als auch zwischen den beiden Krankenhäusern nahezu identisch sind - für die Vergleichbarkeit beider Krankenhäuser eines der wichtigsten Kriterien. 


\subsection{Menge der verabreichten schlafanstoßenden Medikamente}

Der Anteil von Patienten, die schlafanstoßende Medikamente erhielten, ging von 50,3\% (vor der Intervention) auf 36,3\% (nach der Intervention) signifikant zurück. Entsprechend ging auch die Anzahl der Tage, an denen schlafanstoßende Medikamente im Krankenhaus gegeben wurden von 40 Prozent auf rund 30 Prozent zurück.

Die zwei bereits erwähnten Studien liefern uns Zahlen für die Prävalenz von psychotropen Medikamenten (Arnold et al. 2017) beziehungsweise Benzodiazepinen und Z-Substanzen (Heinemann et al. 2019) im selben Krankenhaus, die wir mit Prävalenzen unserer Erhebung vor der Intervention vergleichen können. Arnold et al. (2017) fanden heraus, dass 54\% der Patienten psychotrope Medikamente erhielten. Heinemann et al. (2019) stellten fest, dass 46\% schlafanstoßende Medikamente und 30\% ein Benzodiazepin, eine Z-Substanz oder beides erhielten. Die kleinen Unterschiede in der Prävalenz lassen sich leicht erklären (s.a. Kap. 6.1.2): Bei Arnold et al. (2017) unterschied sich die Zusammensetzung der Wirkstoffliste bezüglich aktivierender Antidepressiva und Neuroleptika. Heinemann et al. (2019) nutzten eine ähnliche Liste an Wirkstoffen, schlossen jedoch Patienten mit der Diagnose Demenz aus - eine Gruppe, bei der ein hoher Hypnotika-Gebrauch bekannt ist (Giron et al. 2001).

Internationale Interventionsstudien, die ebenfalls auf eine Minderung der Verordnung psychotroper Substanzen zielten, ergaben Erfolgsquoten von 5 bis zu 19 Prozentpunkten. Badr et al. (2018) führten in einem amerikanischen Gemeindekrankenhaus eine kontrollierte Interventionsstudie durch, die die Anzahl der Patienten mit einer gleichzeitigen Verordnung mehrerer Sedativa bzw. Hypnotika von 34\% auf 16\% verringerte. Dolan et al. (2012) berichten von einem Audit zur Verschreibung von Benzodiazepinen und Z-Substanzen in einem irischen Krankenhaus mit Feedback und Schulungsangeboten an die niedergelassenen Ärzte der Region. Nach 6 Monaten waren die Verschreibungen von 54\% auf 46\% gefallen. In ihrem nicht-kontrollierten Audit fanden Elliott et al. (2001), dass sich die Interventionen, bestehend aus multidisziplinärem Feedback und Trainings zur Qualität von BenzodiazepinVerschreibungen, auf die Anzahl der Patienten mit Benzodiazepinen in den neun Krankenhäusern der Studie auswirkte: Die Benzodiazepin-Verordnungen sanken von 36\% auf 31\%. Die genannten Studien unterscheiden sich in den einbezogenen Substanzen, in der Studienpopulation und in der Intervention. Bei allen Unterschieden ist aber festzustellen, dass eine Minderung der Verordnung der untersuchten Substanzen erzielbar ist. Der Unterschied von vorher zu nachher mit 14 Prozentpunkten liegt in unserer Studie im höheren Bereich. Die Annahme, dass die Minderung des Anteils der Patienten mit schlafanstoßenden Medikamenten auf das Verschreibungsverhalten der Ärzte zurückzuführen ist, erscheint plausibel. 


\subsection{Angemessenheit der verabreichten schlafanstoßenden Medikamente}

Um die Angemessenheit der Verabreichungen, bzw. deren Änderung besser darzustellen, bietet sich die PRISCUS-Liste an mit ihrer Aufteilung der Medikamente in für ältere Personen angemessene und potenziell inadäquate Medikation. Demnach gab es einen starken Rückgang in der Anzahl der Patienten, die potenziell inadäquate Medikamente erhielten, von 21,5\% vor der Intervention auf nur noch 8,7\% nachher. Rückläufig war auch die Anzahl der Tage mit potenziell inadäquaten Medikamenten. Umgekehrt stieg der Anteil empfohlener Medikamente aus der SOP deutlich an.

Internationale Studien untersuchten im Sinne der Angemessenheit ebenfalls, ob eine Intervention eine Reduktion potenziell inadäquater Medikation bewirken kann. Die Interventionen beziehen sich oftmals auf alle Medikamente PRISCUS-äquivalenter Listen (wie die Beers-Kriterien oder STOPP-Kriterien) (Corbi et al. 2015), auf alle psychotropen Medikamente (Avorn et al. 1992) oder spezifisch auf Benzodiazepine (Pimlott et al. 2003).

Corbi et al. (2015) führten in einer italienischen Rehabilitationsklinik eine Intervention zur Reduktion potenziell inadäquater Medikamente durch. Die Ärzte erhielten ein 3-tägiges Training über potenziell inadäquate Medikation und eine Software, die neue Medikation nach Angemessenheit prüfte. Im Rahmen der Intervention kam es zu einem Rückgang der Patienten mit potenziell inadäquater Medikation von 55\% auf 48\%. Eine frühe Interventionsstudie von Avorn et al. (1992), die sich auf die Gruppe psychotroper Medikamente bezog, wurde in 12 amerikanischen Altenpflegeheimen durchgeführt. Nach der Intervention, einem Schulungsprogramm für das behandelnde Personal, kam es zu einer statistisch signifikanten Reduktion des Gebrauchs psychotroper Medikamente. Pimlott et al. (2003) setzten in Ontario, Kanada eine Intervention um, mit der die Benzodiazepin-Verordnungen von Hausärzten verbessert werden sollten. Als inadäquat wurden hier die gleichzeitige Behandlung eines Patienten mit verschiedenen Benzodiazepinen und der Einsatz lang wirksamer Benzodiazepine gewertet. Die Ärzte erhielten in regelmäßigen Abständen Rückmeldungen über ihr Verschreibungsverhalten und Lehrmaterial über Benzodiazepine. Auch nach dieser Intervention ging der Anteil der inadäquaten Medikamente von 20,3\% auf 19,6\% zurück, wenn auch nicht statistisch signifikant.

Der gleichzeitige Rückgang der Anzahl der Patienten mit schlafanstoßenden Medikamenten und der Anzahl der Tage mit schlafanstoßenden Medikamenten, wie auch der Rückgang der Anzahl der Patienten mit potenziell inadäquater Medikation und der Anzahl der Tage mit potenziell inadäquaten Medikamenten lassen vermuten, dass die Interventionen einen Einfluss auf die Menge und Angemessenheit der schlafanstoßenden Medikamente hatten. Was diese Annahme verstärkt ist der Wechsel von nicht-empfohlenen Medikamentengaben zu in der SOP empfohlenen Medikamentengaben. 


\subsection{Unterschiede in den Fachabteilungen}

Es zeigten sich zum Teil deutliche Unterschiede in Menge und Angemessenheit schlafanstoBender Medikamente zwischen den verschiedenen Fachabteilungen vor und nach der Intervention. Auf den geriatrischen Stationen war der Anteil der Patienten mit schlafanstoßenden Medikamenten sowohl vor, als auch nach der Intervention am höchsten. Neukirchen (2018) und Arnold et al. (2017) berichten vergleichbare Ergebnisse. Dies dürfte durch den größeren Anteil an Patienten mit neurologisch-psychiatrischen Diagnosen und dem damit verbundenen höheren Einsatz psychotroper Medikation zu erklären sein (Abi-Ackel et al. 2017). Neben einer Akutstation gehört zur geriatrischen Abteilung des Krankenhauses auch eine Rehabilitationsstation. Wie auch Neukirchen (2018) ausführt, geht das sich von den anderen Stationen unterscheidende geriatrische Behandlungskonzept mit einer insgesamt längeren Aufenthaltsdauer einher. Eine längere Aufenthaltsdauer wurde in vergangenen Studien mit einem höheren Hypnotika-Gebrauch in Verbindung gebracht (O’Reilly und Rusnak 1990; Somers et al. 2011). Während der Gebrauch von schlafanstoßenden Medikamenten auf geriatrischen Stationen in beiden Zeiträumen hoch war, war der Anteil potenziell inadäquater Medikamente in beiden Zeiträumen niedrig, was auf ein insgesamt angemesseneres Verschreibungsverhalten auf diesen Stationen hinweist.

Auf den chirurgischen Stationen war der Anteil der Patienten mit schlafanstoßenden Medikamenten im ersten Zeitraum verhältnismäßig hoch, sank bis nach den Interventionen aber am stärksten unter den drei Disziplinen. Dies gilt auch für die Angemessenheit der Medikamentengaben. Der anfangs hohe Gebrauch an schlafanstoßenden Medikamenten steht im Kontrast zu der niedrigen Rate an indikationsstellenden Diagnosen und der kurzen Aufenthaltsdauer auf den Stationen, was auf den geriatrischen Stationen eine Erklärung für den hohen Gebrauch darstellen kann. Dieser Unterschied zwischen den beiden Zeiträumen ist bemerkenswert. Wie Leape (2014) in einem Editorial über die Implementierung einer chirurgischen Sicherheits-Checkliste in der Gesundheitsversorgung schreibt, ist die „Geschichte der Patientensicherheitsbewegung eine des langsamen Fortschritts“. Um der Antwort näher zu kommen, was diesen rasanten Fortschritt bewirkt haben kann, wäre eine Befragung der Ärzte der verschiedenen Disziplinen vorstellbar.

\subsection{Schlafanstoßende Medikamente für Patienten mit und ohne neurologisch-psychiatrische Diagnosen}

Durch die Intervention sollte vor allem die Gruppe der Patienten obne neurologisch-psychiatrische Diagnosen erreicht werden, denn gerade bei diesen Patienten besteht möglicherweise keine Indikation für den Einsatz schlafanstoßender Medikamente. Umgekehrt handelt es sich bei der Gruppe mit neurologisch-psychiatrischen Diagnosen um Patienten, denen notwendige Psychopharmaka nicht verweigert werden sollten. In der Gruppe der Patienten mit neurologisch-psychiatrischen Diagnosen war der Anteil, der schlafanstoßende Medika- 
mente erhielt, größer als in der Gruppe der Patienten ohne solche Diagnosen. Gleichermaßen höher war in dieser Gruppe die Anzahl der Tage mit schlafanstoßenden Medikamenten, sowohl vor als auch nach der Intervention. Es erscheint logisch, dass der Bedarf für Psychopharmaka in dieser Patientengruppe insgesamt höher ist. Die Menge der Verabreichungen schlafanstoßender Medikamente ging für Patienten mit neurologisch-psychiatrischen Diagnosen zum zweiten Zeitraum hin in einem geringeren Maße zurück als für die Gruppe der Patienten ohne derartige Diagnosen. Dies spricht dafür, dass die Patienten mit neurologischpsychiatrischen Diagnosen im Rahmen der Intervention nicht unterversorgt wurden, also ihnen notwendige Psychopharmaka verweigert wurden. In dieser Patientengruppe ist auch zu sehen, dass der Anteil, der mit potenziell inadäquaten Medikamenten versorgt wurde, von 21,2\% auf 11,5\% der Patienten fiel. Entsprechend rückläufig war auch die Anzahl der Tage mit potenziell inadäquaten Medikamenten. Das Verhältnis von Gaben von in der SOP empfohlenen Medikamenten stieg an. Zusammenfassend wurden Patienten mit neurologischpsychiatrischen Diagnosen im Rahmen der Intervention nicht unterversorgt, der Anteil potenziell inadäquater Medikamente verringerte sich und die Angemessenheit der Medikamentengaben verbesserte sich.

In der Gruppe der Patienten ohne neurologisch-psychiatrische Diagnosen fiel der Unterschied sowohl in Menge als auch Angemessenheit der schlafanstoßenden Medikamente deutlich größer aus (um das Dreifache der Prozentpunkte in der Anzahl der Patienten mit schlafanstoßenden Medikamenten und um das Eineinhalbfache der Prozentpunkte mehr bei den Patienten mit potenziell inadäquaten Medikamenten). Unterschiede sind also vor allem in der eigentlichen Zielgruppe der Intervention zu beobachten, den Patienten, die keine neurologisch-psychiatrische Diagnose und keine Indikation für schlafanstoßende Medikamente hatten.

Es ist nicht auszuschließen, dass dennoch Patienten angemessene Hypnotika/Sedativa nicht erhielten, weil die Intervention hier und dort „,̈̈ber das Ziel hinausschoss“. Hierüber könnten Einzelbefragungen von Patienten mit neurologisch-psychiatrischen Erkrankungen Auskunft geben, um gegebenenfalls einer zu weit gehenden Absetzung von Psychopharmaka durch diese oder ähnliche Interventionen entgegenzuwirken. 


\subsection{Häufigkeit der verschiedenen Wirkstoffe}

Im Folgenden soll der in Kapitel 6.2 beschriebene Rückgang in der Menge schlafanstoßender Medikamente noch einmal detaillierter nach den einzelnen Wirkstoffen untersucht werden. Bei dieser Aufspaltung der einzelnen Wirkstoffgruppen ist der starke signifikante Rückgang von Benzodiazepinen auffällig; bei den Z-Substanzen war ein leichter, ebenfalls signifikanter, Rückgang zu verzeichnen. Der Gebrauch von Neuroleptika und Antidepressiva blieb annähernd gleich.

Die Ergebnisse unserer Erhebung sind ähnlich auch auf nationaler Ebene im Arzneiverordnungs-Report zu finden. Im Zeitraum 2013 bis 2017 ist der Einsatz von Benzodiazepinen und Z-Substanzen rückläufig, während Neuroleptika und Antidepressiva ungefähr gleichbleibend häufig verordnet werden. Auch bei genauerer Betrachtung der verschiedenen Wirkstoffe innerhalb der jeweiligen Wirkstoffgruppe ähneln unsere Ergebnisse denen im Arzneiverordnungs-Report. So nehmen Verordnungen sog. atypischer Neuroleptika (Quetiapin, Risperidon) zu, während die niedrig- und hochpotenten Neuroleptika ungefähr gleichbleibend oder abnehmend verordnet werden (Melperon, Prothipendyl, Promethazin, Haloperidol). Die Verordnung trizyklischer Antidepressiva sinkt (Amitriptylin, Doxepin), die Verordnungen von Mirtazapin bleiben ungefähr gleich (Lohse und Müller-Oerlinghausen 2014a; Lohse und Müller-Oerlinghausen 2014b; Lohse und Müller-Oerlinghausen 2018b; Lohse und Müller-Oerlinghausen 2018a). Die Autoren erwähnen, dass der Anteil der Patienten, der - epidemiologischen Zahlen zufolge - an Schlafproblemen leidet, bedeutend größer sei als der Anteil der Patienten, der mit Hypnotika (Benzodiazepine und Z-Substanzen) behandelt wird. Sie äußern die Vermutung, dass viele Patienten mit Schlafproblemen entweder keine ärztliche Behandlung suchen oder anstatt von Hypnotika mit sedierenden Antidepressiva oder Neuroleptika behandelt würden (Lohse und Müller-Oerlinghausen 2018a, S. 508). Bei den Zahlen des Arzneiverordnungs-Reports sind zwei Aspekte zu beachten: (1) Sie stellen die ambulante Patientenversorgung dar, nicht die Krankenhausversorgung. (2) Wahrscheinlich werden die Zahlen der Hypnotika unterschätzt, da viele auf Privatrezept verordnet werden (Hoffmann und Glaeske 2014).

Auch internationale Studien, die den Gebrauch psychotroper Medikamente über einen längeren Zeitraum betrachteten, fanden Trends, die denen im Arzneiverordnungs-Report ähnelten: So ging in einer Beobachtungsstudie von Patienten mit Demenz, die in Altenpflegeheimen (Ontario, Kanada) wohnen, der Gebrauch psychotroper Medikamente von 2004 bis 2013 insgesamt etwas zurück (Vasudev et al. 2015). Die einzelnen Wirkstoffgruppen verhielten sich unterschiedlich: Benzodiazepine wurden weniger häufig genutzt, der Einsatz von Antipsychotika blieb hoch und der von Antidepressiva stieg an. Dieses Muster suggeriere, laut den Autoren, dass Antidepressiva und Antipsychotika Benzodiazepine in ihrer Rolle als sedierende Medikamente ersetzen. Pitkala et al. (2015) untersuchten die Prävalenz von Opiaten, Analgetika und psychotropen Medikamenten über 8 Jahre hinweg bei älteren Patienten in finnischen Altenpflegeheimen und Einrichtungen für betreutes Wohnen. Auch wurden 
Hypnotika und Anxiolytika möglicherweise durch andere Medikamente mit sedierenden Eigenschaften ersetzt, wie Opiate, Pregabalin, Gabapentin und sedierende Antidepressiva.

Arnold et al. (2017, S. 7) bestätigen ein solches Verordnungsverhalten zumindest für die geriatrischen Stationen unseres Erhebungskrankenhauses. Dort wurden offensichtlich im Zeitraum vor der Intervention Antidepressiva und Neuroleptika als Ersatz für Benzodiazepine und Z-Substanzen zur Schlafinduktion eingesetzt.

Während der Rückgang von Benzodiazepinen und Zopiclon zwar Empfehlungen der Intervention entspricht, gab es wohl keinen Austausch von Benzodiazepinen durch weniger problematische Medikamente. Die Ergebnisse der zuvor genannten Studien und das steigende Bewusstsein für die negativen Wirkungen von Benzodiazepinen lassen einen solchen Wandel im Gesundheitssystem allerdings erwarten (Kruse 1990; Lader 2011). Nicht zuletzt gibt es Empfehlungen über den Einsatz von Antidepressiva und Neuroleptika spezifisch als Hypnotika (Enzensperger und Lehmann 2007). Auch wenn bei Medikamenten aus den Gruppen der Neuroleptika und Antidepressiva gravierende Nebenwirkungen auftreten können, spräche ein solcher Wechsel für die vermehrte Nutzung von Medikamentengruppen, die als adäquat für ältere Patienten gelten. Um - über unsere Ergebnisse hinaus - herauszufinden, ob im Krankenhaus Weende anstatt Benzodiazepine und Z-Substanzen tatsächlich andere Medikamente als Schlafmittel eingesetzt werden, wäre eine Befragung der Ärzte und Pfleger erforderlich.

\subsubsection{Z-Substanzen}

Die Verordnungen von Z-Substanzen sind insgesamt zurückgegangen, nach Wirkstoffen allerdings unterschiedlich. Während vor der Intervention Zopiclon häufiger gegeben wurde als Zolpidem, war es nach der Intervention umgekehrt. Das Bild vor der Intervention entspricht den Verordnungshäufigkeiten im Arzneiverordnungsreport, nach der Intervention allerdings nicht mehr. Der Trend könnte ein Hinweis darauf sein, dass die Empfehlung in der SOP, Zolpidem (in einer Dosierung $\leq 5 \mathrm{mg}$ ) zu nutzen, Anklang gefunden hat.

\subsubsection{Baldrian}

Bei Baldrian - als pflanzliches Mittel und als empfohlene Alternative in der SOP - würde man spontan vielleicht hoffen und erwarten, dass es nach der Intervention häufiger eingesetzt würde. Eine Abnahme der stärkeren schlafanstoßenden Medikamente mit einem gleichzeitigen Anstieg der Baldriannutzung scheint doch ganz im Sinne der Intervention, da es ein weniger potenziell gefährliches Medikament darstellt. Auf der anderen Seite war es kein Ziel der Intervention, Menschen mit Schlafproblemen zu dem falschen Schluss zu bringen, dass diese primär mit der Einnahme von Tabletten zu lösen sind. Aus diesen Gründen haben wir den Anteil der Patienten, die schlafanstoßende Medikamente erhielten, sowohl mit als auch ohne Baldrian ausgewertet. 
Erfreulicherweise gab es keine Zunahme der Patienten, die Baldrian erhielten - ihr Anteil ging von rund $11 \%$ vor der Intervention auf $7 \%$ nach der Intervention zurück. Entsprechend rückläufig war auch die Anzahl der Patienten mit allen schlafanstoßenden Medikamenten sowohl unter Einbezug von Baldrian, als auch ohne.

Der Effekt, dass Baldrian andere stärkere schlafanstoßende Medikamente ersetzt, ist offensichtlich nicht eingetreten. Vielmehr reiht sich Baldrian ein in die anderen schlafanstoßenden Medikamente, die nach der Intervention weniger verabreicht wurden.

\subsection{Vergleich mit dem Kontrollkrankenhaus}

Im Kontrollkrankenhaus war der Anteil der Patienten, die schlafanstoßende Medikamente erhielten, im ersten Zeitraum geringer als im Interventionskrankenhaus. Dort erhielten 43,2\% (158/366) der Patienten schlafanstoßende Medikamente; im Interventionskrankenhaus 50,3\% (493/960). Über die Gründe für diesen Unterschied kann keine Aussage getroffen werden - was auch nicht das Thema der vorliegenden Arbeit ist.

Wie im Interventionskrankenhaus war auch im Kontrollkrankenhaus ein Rückgang in der Menge der verbreichten schlafanstoßenden Medikamente zu sehen. Im Interventionskrankenhaus fiel der Anteil der Patienten mit schlafanstoßenden Medikamenten statistisch signifikant von 50,3\% auf 36,3\%, also um 14 Prozentpunkte - im Kontrollkrankenhaus von 43,2\% auf 36,5\% (6 Prozentpunkte, nicht signifikant). Ein Rückgang war also in beiden Krankenhäusern zu sehen, er fiel allerdings deutlich unterschiedlich zugunsten des Interventionskrankenhauses aus.

Dieser Rückgang spricht für einen Nutzen der Intervention in dem Interventionskrankenhaus - kritisch ist allerdings anzumerken, dass dieses Krankenhaus auf ein Niveau gehoben werden konnte, welches das Kontrollkrankenhaus bereits ohne eine Intervention erreicht hatte.

Von den Patienten ohne neurologisch-psychiatrische Diagnosen erhielten im Kontrollkrankenhaus 35,4\% schlafanstoßende Medikamente im ersten Zeitraum, 27,4\% im zweiten Zeitraum. Von den Patienten mit diesen Diagnosen erhielten 65,3\% schlafanstoßende Medikamente im ersten und 60,7\% im zweiten Zeitraum. Der Rückgang lag also bei 8,0 Prozentpunkten bei Patienten ohne und knapp 5 Prozentpunkten bei Patienten mit neurologischpsychiatrischen Diagnosen. Vor der Intervention erhielten im Interventionskrankenhaus 45,0\% der Patienten ohne neurologisch-psychiatrische Diagnosen entsprechende Medikamente, danach 28,8\%. Bei den Patienten mit neurologisch-psychiatrischen Diagnosen waren es 67,1\% vorher und 62,4\% nachher. Der Rückgang in der Menge schlafanstoßender Medikamente war also in der Zielgruppe der Intervention, den Patienten ohne neurologisch-psychiatrische Diagnosen, deutlich stärker ausgeprägt - 16 Prozentpunkte - verglichen mit knapp 5 Prozentpunkten bei den Patienten mit neurologisch-psychiatrischen Diagnosen. Die 
Unterschiede zwischen den beiden Zeiträumen fielen im Interventionskrankenhaus deutlich klarer aus, was für einen Erfolg der Intervention spricht.

\subsection{Schlussfolgerungen}

Eine auf mehreren Ebenen ansetzende Intervention führte zu einem signifikanten Rückgang der verabreichten schlafanstoßenden Medikamente insgesamt als auch einem Rückgang potenziell inadäquater Verabreichungen. Gleichzeitig wurden - für ältere Patienten - besser geeignete Medikamente, die in einer Leitlinie der Intervention genannt wurden, nach der Intervention häufiger genutzt.

Die Intervention zielte zwar auf eine Reduktion schlafanstoßender Medikamente für Patienten ohne neurologisch-psychiatrische Diagnosen. Sie hätte allerdings auch dazu führen können, dass Patienten, die möglicherweise entsprechende Medikamente benötigen, unterversorgt werden. Dies scheint nicht eingetroffen zu sein.

Bei der Betrachtung der einzelnen Wirkstoffe fällt auf, dass vor allem Verabreichungen von Benzodiazepinen und Z-Substanzen signifikant zurückgingen - entsprechend den Empfehlungen, die im Rahmen der Intervention gegeben wurden.

Ein genereller (,,säkulärer") Trend, weniger schlafanstoßende Medikamente zu verschreiben, lässt sich bei einem Vergleich mit dem Kontrollkrankenhaus feststellen. Der Rückgang im Interventionskrankenhaus bleibt deutlich klarer.

Die Ergebnisse stimmen mit Übersichtsarbeiten überein, welche die Effektivität von Interventionen belegen, die auf mehreren Ebenen ansetzen (Smith und Tett 2010; Mostofian et al. 2015; Rankin et al. 2018). Dies bestärkt die Annahme, dass ein kausaler Zusammenhang zwischen der Intervention und dem Rückgang schlafanstoßender Medikamente besteht.

Die Aussagekraft der Ergebnisse ist eingeschränkt durch das Studiendesign. Sicherere Rückschlüsse über die Effektivität der stattgefundenen Intervention wären mit einem Clusterrandomisierten Design möglich, in dem mehrere Krankenhäuser bzw. Stationen zufällig einer Interventions- bzw. Kontrollgruppe zugeordnet werden.

Für die Zukunft könnte sich der Einsatz von elektronischen Arzneiverordnungssystemen, in denen potenziell inadäquate Verordnungen zum Zeitpunkt der Verordnung festgestellt und direkt rückgemeldet werden, als vielversprechend erweisen (Gurwitz und Rochon 2002; O’Connor et al. 2012; Rankin et al. 2018). Die Ergänzung der Mehrebenen-Intervention mit dieser Technik (welche allerdings bisher nicht flächendeckend in Krankenhäusern vorhanden ist) und einer Überprüfung anhand eines randomisierten-kontrollieren Studiendesigns wäre ein vorstellbarer nächster Schritt. 


\section{$7 \quad$ Zusammenfassung}

Hintergrund. Bei Ein- und Durchschlafproblemen im Krankenhaus erhalten Patienten oftmals schlafanstoßende Medikamente, möglicherweise mit erheblichen Nebenwirkungen, insbesondere für ältere Patienten. Eine auf mehreren Ebenen eingreifende Intervention sollte den Gebrauch dieser Medikamente einschränken bzw. rationaler gestalten.

Fragestellung. Hat die Intervention die Menge der schlafanstoßenden Medikamente für ältere Patienten reduziert und die Angemessenheit ihrer Anwendung verbessert?

Methode. Im Rahmen einer nicht-randomisierten kontrollierten Studie mit einem Prä-Postdesign („Quasi-Experiment") wurden Anzahl, Art und Angemessenheit der eingesetzten schlafanstoßenden Medikamente in einem Interventions- und einem Kontrollkrankenhaus verglichen. Die Medikamente wurden anhand der sog. PRISCUS-Liste in angemessene oder potenziell unangemessene Medikamente eingeteilt.

Ergebnisse. Im Interventionskrankenhaus wurden 960 Patientenakten aus dem Zeitraum vor der Intervention (Juli/August 2013) und 1043 Akten nach der Intervention (Juli/August 2017) ausgewertet. Der Anteil der Patienten, der schlafanstoßende Medikamente erhielt, ging signifikant um 14 Prozentpunkte von 50,3\% auf 36,3\% zurück. Die Zahl der Patienten, die mindestens einmal ein potenziell unangemessenes Medikament erhielten, verringerte sich von $21,5 \%$ auf $8,7 \%$. Der Anteil der in der Interventions-Leitlinie empfohlenen Medikamente stieg von 47,2\% auf 58,5\%. Im Kontrollkrankenhaus erhielten im Zeitraum Juli/August 2013 43,2\% der Patienten (366 ausgewertete Akten) schlafanstoßende Medikamente, im Zeitraum Juli/August 2017 36,5\% (411 Akten), also eine Reduktion um 7 Prozentpunkte.

Diskussion. Sowohl im Interventions- als auch im Kontrollkrankenhaus ging die Verabreichung schlafanstoßender und potenziell inadäquater Medikamente vom ersten zum zweiten Zeitraum zurück. Der Unterschied zwischen den beiden Zeiträumen fällt im Interventionskrankenhaus deutlich klarer aus (14 vs. 7 Prozentpunkte), was für eine Interventions-bedingte Änderung spricht. Das gilt besonders für die Benzodiazepine. Das Ergebnis ist vereinbar mit Erkenntnissen aus Übersichtsarbeiten, wonach komplexe Interventionen zu Verbesserungen in der Patientenversorgung führen. Verbindliche Rückschlüsse über den Einfluss der Intervention auf die Veränderungen der medikamentösen Versorgung im Studienkrankenhaus sind durch das quasi-experimentelle Studiendesign limitiert - eine (cluster-)randomisierte Untersuchung mehrerer Stationen bzw. Krankenhäuser wäre wünschenswert. 


\section{$8 \quad$ Anhang}

\subsection{Wirkstoffliste}

\begin{tabular}{|c|c|c|c|c|c|}
\hline Nr. & Wirkstoffgruppe & $\begin{array}{l}\text { Wirkstoff- } \\
\text { name }\end{array}$ & $\begin{array}{c}\text { Auf } \\
\text { PRISCUS } \\
\text { Liste }^{*}\end{array}$ & $\begin{array}{l}\text { Potenziell } \\
\text { inadäquate } \\
\text { Dosierung } \\
\text { (PRISCUS)† }\end{array}$ & $\begin{array}{l}\text { Handels- } \\
\text { namen }\end{array}$ \\
\hline 1 & $\begin{array}{l}\text { Andere Schlaf- und } \\
\text { Beruhigungsmittel }\end{array}$ & Baldrian & Nein & - & \\
\hline 2 & $\begin{array}{l}\text { Andere Schlaf- und } \\
\text { Beruhigungsmittel }\end{array}$ & Chloralhydrat & $\mathrm{Ja}$ & - & \\
\hline 3 & $\begin{array}{l}\text { Andere Schlaf- und } \\
\text { Beruhigungsmittel }\end{array}$ & Clomethiazol & Nein & - & \\
\hline 4 & $\begin{array}{l}\text { Andere Schlaf- und } \\
\text { Beruhigungsmittel }\end{array}$ & $\begin{array}{l}\text { Diphenhydra- } \\
\min \end{array}$ & $\mathrm{Ja}$ & - & \\
\hline 5 & $\begin{array}{l}\text { Andere Schlaf- und } \\
\text { Beruhigungsmittel }\end{array}$ & Doxylamin & $\mathrm{Ja}$ & - & \\
\hline 6 & $\begin{array}{l}\text { Andere Schlaf- und } \\
\text { Beruhigungsmittel }\end{array}$ & Melatonin & Nein & - & Circadin \\
\hline 7 & Antidepressiva & Agomelatin & Nein & - & \\
\hline 8 & Antidepressiva & Amitriptylin & $\mathrm{Ja}$ & - & Saroten \\
\hline 9 & Antidepressiva & Doxepin & $\mathrm{Ja}$ & - & Aponal \\
\hline 10 & Antidepressiva & Maprotilin & $\mathrm{Ja}$ & - & \\
\hline 11 & Antidepressiva & Mianserin & Nein & - & \\
\hline 12 & Antidepressiva & Mirtazapin & Nein & - & Remergil \\
\hline 13 & Antidepressiva & Opipramol & Nein & - & Insidon \\
\hline 14 & Antidepressiva & Trazodon & Nein & - & \\
\hline 15 & Antidepressiva & Trimipramin & $\mathrm{Ja}$ & - & Stangyl \\
\hline 16 & $\begin{array}{l}\text { Benzodiazepine - kurz } \\
\text { und mittellang wirksam }\end{array}$ & Alprazolam & $\mathrm{Ja}$ & - & \\
\hline 17 & $\begin{array}{l}\text { Benzodiazepine - kurz } \\
\text { und mittellang wirksam }\end{array}$ & Brotizolam & $\mathrm{Ja}$ & $>0,125 \mathrm{mg} / \mathrm{Tag}$ & \\
\hline 18 & $\begin{array}{l}\text { Benzodiazepine - kurz } \\
\text { und mittellang wirksam }\end{array}$ & Lorazepam & $\mathrm{Ja}$ & $>2 \mathrm{mg} / \mathrm{Tag}$ & Tavor \\
\hline 19 & $\begin{array}{l}\text { Benzodiazepine - kurz } \\
\text { und mittellang wirksam }\end{array}$ & $\begin{array}{l}\text { Lorme- } \\
\text { tazepam }\end{array}$ & $\mathrm{Ja}$ & $>0,5 \mathrm{mg} / \mathrm{Tag}$ & Noctamid \\
\hline 20 & $\begin{array}{l}\text { Benzodiazepine - kurz } \\
\text { und mittellang wirksam }\end{array}$ & Nitrazepam & Nein & - & \\
\hline 21 & $\begin{array}{l}\text { Benzodiazepine - kurz } \\
\text { und mittellang wirksam }\end{array}$ & Oxazepam & $\mathrm{Ja}$ & $>60 \mathrm{mg} / \mathrm{Tag}$ & $\begin{array}{l}\text { Adumbran, } \\
\text { Durazepam }\end{array}$ \\
\hline
\end{tabular}

* Medikamente, die auf der PRISCUS-Liste als potenziell inadäquate Medikamente für ältere Patienten eingeordnet werden.

† Medikamente, die erst ab einer bestimmten Dosierung als potenziell inadäquate Medikamente für ältere Patienten eingeordnet werden. 


\begin{tabular}{|c|c|c|c|c|c|}
\hline Nr. & Wirkstoffgruppe & $\begin{array}{l}\text { Wirkstoff- } \\
\text { name }\end{array}$ & $\begin{array}{c}\text { Auf } \\
\text { PRISCUS } \\
\text { Liste }^{*}\end{array}$ & $\begin{array}{c}\text { Potenziell } \\
\text { inadäquate } \\
\text { Dosierung } \\
\text { (PRISCUS)† }\end{array}$ & $\begin{array}{l}\text { Handels- } \\
\text { namen }\end{array}$ \\
\hline 22 & $\begin{array}{l}\text { Benzodiazepine - kurz } \\
\text { und mittellang wirksam }\end{array}$ & Temazepam & $\mathrm{Ja}$ & - & $\begin{array}{l}\text { Planum, } \\
\text { Remestan }\end{array}$ \\
\hline 23 & $\begin{array}{l}\text { Benzodiazepine - kurz } \\
\text { und mittellang wirksam }\end{array}$ & Tetrazepam & $\mathrm{Ja}$ & - & Musaril \\
\hline 24 & $\begin{array}{l}\text { Benzodiazepine - kurz } \\
\text { und mittellang wirksam }\end{array}$ & Triazolam & $\mathrm{Ja}$ & - & \\
\hline 25 & $\begin{array}{l}\text { Benzodiazepine - } \\
\text { lang wirksam }\end{array}$ & Bromazepam & $\mathrm{Ja}$ & - & \\
\hline 26 & $\begin{array}{l}\text { Benzodiazepine - } \\
\text { lang wirksam }\end{array}$ & $\begin{array}{l}\text { Chlordia- } \\
\text { zepoxid }\end{array}$ & $\mathrm{Ja}$ & - & \\
\hline 27 & $\begin{array}{l}\text { Benzodiazepine - } \\
\text { lang wirksam }\end{array}$ & Clobazam & $\mathrm{Ja}$ & - & \\
\hline 28 & $\begin{array}{l}\text { Benzodiazepine - } \\
\text { lang wirksam }\end{array}$ & Diazepam & $\mathrm{Ja}$ & - & \\
\hline 29 & $\begin{array}{l}\text { Benzodiazepine - } \\
\text { lang wirksam }\end{array}$ & $\begin{array}{l}\text { Dikaliumclo- } \\
\text { razepat }\end{array}$ & $\mathrm{Ja}$ & - & Tranxilium \\
\hline 30 & $\begin{array}{l}\text { Benzodiazepine - } \\
\text { lang wirksam }\end{array}$ & $\begin{array}{l}\text { Flunitra- } \\
\text { zepam }\end{array}$ & $\mathrm{Ja}$ & - & Rohypnol \\
\hline 31 & $\begin{array}{l}\text { Benzodiazepine - } \\
\text { lang wirksam }\end{array}$ & Flurazepam & $\mathrm{Ja}$ & - & $\begin{array}{l}\text { Dalma- } \\
\text { dorm, Stau- } \\
\text { rodorm }\end{array}$ \\
\hline 32 & $\begin{array}{l}\text { Benzodiazepine - } \\
\text { lang wirksam }\end{array}$ & Medazepam & $\mathrm{Ja}$ & - & \\
\hline 33 & $\begin{array}{l}\text { Benzodiazepine - } \\
\text { lang wirksam }\end{array}$ & Prazepam & $\mathrm{Ja}$ & - & \\
\hline 34 & Neuroleptika & Aripiprazol & Nein & - & \\
\hline 35 & Neuroleptika & Asenapin & Nein & - & \\
\hline 36 & Neuroleptika & Benperidol & Nein & - & Glianimon \\
\hline 37 & Neuroleptika & $\begin{array}{l}\text { Chlorprothi- } \\
\text { xen }\end{array}$ & Nein & - & \\
\hline 38 & Neuroleptika & Clozapin & $\mathrm{Ja}$ & - & \\
\hline 39 & Neuroleptika & Flupentixol & Nein & - & \\
\hline 40 & Neuroleptika & Fluphenazin & $\mathrm{Ja}$ & - & \\
\hline 41 & Neuroleptika & Haloperidol & $\mathrm{Ja}$ & $>2 \mathrm{mg}$ & \\
\hline 42 & Neuroleptika & $\begin{array}{l}\text { Levome- } \\
\text { promazin }\end{array}$ & $\mathrm{Ja}$ & - & \\
\hline 43 & Neuroleptika & Melperon & Nein & - & $\begin{array}{l}\text { Eunerpan, } \\
\text { Melneurin }\end{array}$ \\
\hline 44 & Neuroleptika & Olanzapin & $\mathrm{Ja}$ & $>10 \mathrm{mg}$ & \\
\hline 45 & Neuroleptika & Perazin & Nein & - & \\
\hline 46 & Neuroleptika & Perphenazin & Nein & - & \\
\hline 47 & Neuroleptika & Pipamperon & Nein & - & \\
\hline
\end{tabular}




\begin{tabular}{|c|c|c|c|c|c|}
\hline Nr. & Wirkstoffgruppe & $\begin{array}{l}\text { Wirkstoff- } \\
\text { name }\end{array}$ & $\begin{array}{c}\text { Auf } \\
\text { PRISCUS } \\
\text { Liste }^{*}\end{array}$ & $\begin{array}{c}\text { Potenziell } \\
\text { inadäquate } \\
\text { Dosierung } \\
\text { (PRISCUS) }\end{array}$ & $\begin{array}{l}\text { Handels- } \\
\text { namen }\end{array}$ \\
\hline 48 & Neuroleptika & Promethazin & Nein & - & Atosil \\
\hline 49 & Neuroleptika & Prothipendyl & Nein & - & Dominal \\
\hline 50 & Neuroleptika & Quetiapin & Nein & - & Seroquel \\
\hline 51 & Neuroleptika & Risperidon & Nein & - & \\
\hline 52 & Neuroleptika & Thioridazin & $\mathrm{Ja}$ & - & \\
\hline 53 & Neuroleptika & $\begin{array}{l}\text { Zuclopent- } \\
\text { hixol }\end{array}$ & Nein & - & \\
\hline 54 & $\begin{array}{l}\text { Nicht einberechnen } \\
\text { (OP-Medikation) }\end{array}$ & Midazolam & Nein & - & \\
\hline 55 & Z-Substanzen & Zaleplon & $\mathrm{Ja}$ & $>5 \mathrm{mg} / \mathrm{Tag}$ & \\
\hline 56 & Z-Substanzen & Zolpidem & $\mathrm{Ja}$ & $>5 \mathrm{mg} / \mathrm{Tag}$ & Stilnox \\
\hline 57 & Z-Substanzen & Zopiclon & $\mathrm{Ja}$ & $>3,75 \mathrm{mg} / \mathrm{Tag}$ & Ximovan \\
\hline
\end{tabular}




\subsection{Erstellen der Wirkstoffliste}

Basierend auf (1) einer Liste aus einer vorhergehenden Untersuchung von Arnold (2017), (2) Lieferungslisten des Krankenhauses und (3) Fachinformationen, wurde eine Wirkstoffliste mit schlafanstoßenden Medikamenten erstellt.

Anfangs wurde mit einer Wirkstoffliste psychotroper Medikamente aus der vorangegangenen Untersuchung von Arnold (2017) gearbeitet. Aus dieser Liste wurden Hypnotika und Substanzen aus anderen Wirkstoffgruppen, die eine sedierende Wirkung haben und deswegen potenziell als Hypnotikum genutzt werden könnten, übernommen. Nach Rücksprache mit der Apotheke, die zum Zeitpunkt der Erhebungszeiträume 2013 und 2017 das Krankenhaus Weende versorgte, erhielten wir eine Liste aller gelieferten Psychopharmaka der beiden Zeiträume.

\subsubsection{Definition der Hypnotika und Zusammenführen der Informationen}

Grundlage für die Definition von Hypnotika waren Abschnitte aus dem „Kompendium der psychiatrischen Pharmakotherapie“ (Benkert und Hippius 2019; Müller und Benkert 2019; Regen und Benkert 2019; Steiger et al. 2019). Der Arzneiverordnungsreport (Schwabe et al. 2017) half bei der Einschätzung, wie oft ein Medikament in der Erhebung auftauchen könnte. Alle Medikamente, die als Hypnotikum im „Kompendium der psychiatrischen Pharmakotherapie" definiert waren, wurden in die Wirkstoffliste übernommen. Medikamente, die nicht primär als Hypnotikum definiert sind, aber sedierende Eigenschaften haben, sollten auch erhoben werden. Die in Tabelle A 1 dargestellten Wirkmechanismen, die für die sedierenden Eigenschaften von Hypnotika verantwortlich sind, sind auch in manchen Antidepressiva und Antipsychotika zu finden, die nicht primär als Hypnotika definiert sind. Hatten Antidepressiva und Antipsychotika solche Eigenschaften, sollten sie mit auf die Liste der zu erhebenden Wirkstoffe gesetzt werden. Dafür wurden Informationen genutzt, die in Tabelle A 2, Tabelle A 3und Tabelle A 4 dargestellt sind. Medikamente, die in Tabelle A 2 und Tabelle A 4 in einer der Spalten mit zwei „,+ oder mehr, bzw. in Tabelle A 3 mit einem „,+ oder mehr vermerkt sind, wurden in der Wirkstoffliste aufgenommen.

\subsubsection{Vergleich anderer Studien mit Hypnotika}

Um zu vermeiden, dass in der Wirkstoffliste für die vorliegende Untersuchung fälschlich viele Medikamente vorkommen, die in keiner anderen Studie als Hypnotikum definiert werden, wurde eine Übersicht erstellt. In der Übersicht ist zu sehen, welche Wirkstoffe in anderen Studien erhoben wurden, verglichen mit unserer Erhebung (s. Anhang 8.3). 


\subsubsection{Reflektion mit Klinikärzten}

Das Verfahren beim Erstellen der Wirkstoffliste und der Vergleich mit Wirkstoffen aus anderen Studien wurde in einem Gespräch mit zwei klinisch tätigen Ärzten reflektiert. Die vollständige Liste der Wirkstoffe, die erhoben wurden, ist im Anhang 8.1 zu finden.

Tabelle A 1 Wirkmechanismen von Hypnotika. In Anlehnung an Steiger et al. (2019)

\begin{tabular}{|l|l|}
\hline $\begin{array}{l}\text { BZD-Hypnotika } \\
\text { \& Non-BZD- } \\
\text { Hypnotika }\end{array}$ & $\begin{array}{l}\text { Die als Hypnotika gebräuchlichen BZD vermitteln ihre Wirkung } \\
\text { hauptsächlich über die die Untereinheit } \alpha_{1} \text { enthaltenden } \mathbf{G A B A}_{\mathbf{A}^{-}} \\
\text {Rezeptoren. }\end{array}$ \\
\hline Antihistaminika & $\begin{array}{l}\text { Vermittlung der schlaffördernden Wirkung über Blockade zentra- } \\
\text { ler } \mathbf{H}_{1} \text {-Rezeptoren. }\end{array}$ \\
\hline Antidepressiva & $\begin{array}{l}\text { Vermittlung der schlafinduzierenden Wirkung vorwiegend über 5- } \\
\mathbf{H T}_{2}-\text { und } \mathbf{H}_{1} \text {-antagonistische Eigenschaften, z. T. auch über } \\
\text { Blockade der } \boldsymbol{\alpha}_{1} \text {-adrenergen Rezeptoren. }\end{array}$ \\
\hline Antipsychotika & $\begin{array}{l}\text { Vermittlung der schlafinduzierenden Wirkung vorwiegend über 5- } \\
\mathbf{H T}_{2} \text { - und } \mathbf{H}_{1} \text {-antagonistische Eigenschaften, z. T. auch über } \\
\text { Blockade der } \boldsymbol{\alpha}_{1} \text {-adrenergen Rezeptoren. }\end{array}$ \\
\hline \multicolumn{2}{|l|}{ GABA $=\gamma$-Aminobuttersäure, $H=$ Histamin, 5-HT= Serotonin } \\
\hline
\end{tabular}

Mit freundlicher Genehmigung des Springer Nature Verlages 
Tabelle A 2 Übersicht der pharmakologischen Angriffspunkte von Antidepressiva. In Anlehnung an Regen und Benkert (2019)

\begin{tabular}{|c|c|c|c|}
\hline Antidepressivum & $\mathbf{H}_{1}$ & $5-\mathbf{H T}_{2}$ & $\alpha_{1}$ \\
\hline Agomelatin* & 0 & ++ & 0 \\
\hline Amitriptylin & +++ & ++ & +++ \\
\hline Amitriptylinoxid & ++ & ++ & ++ \\
\hline Bupropion & $+/-$ & $+/-$ & + \\
\hline Citalopram & + & 0 & $+/-$ \\
\hline Clomipramin & + & + & ++ \\
\hline Doxepin & +++ & ++ & +++ \\
\hline Duloxetin & $+/-$ & 0 & $+/-$ \\
\hline Escitalopram & $+/-$ & 0 & $+/-$ \\
\hline Fluoxetin & $+/-$ & + & $+/-$ \\
\hline Fluvoxamin & 0 & 0 & $+/-$ \\
\hline Hypericum & 0 & $?$ & 0 \\
\hline Imipramin & $+/-$ & + & + \\
\hline Maprotilin & +++ & + & + \\
\hline Milnacipran & 0 & 0 & 0 \\
\hline Mirtazapin & +++ & ++ & + \\
\hline Moclobemid & 0 & 0 & 0 \\
\hline Paroxetin & 0 & 0 & $+/-$ \\
\hline Reboxetin & 0 & 0 & 0 \\
\hline Sertralin & 0 & 0 & 0 \\
\hline Tianeptin & 0 & 0 & 0 \\
\hline Tranylcypromin & 0 & 0 & 0 \\
\hline Trazodon & + & ++ & ++ \\
\hline Trimipramin & +++ & ++ & +++ \\
\hline Venlafaxin & 0 & 0 & 0 \\
\hline Vortioxetin & 0 & 0 & 0 \\
\hline \multicolumn{4}{|c|}{$\begin{array}{l}H_{1} \text { Antagonismus an Histaminrezeptoren (Typ 1), 5-HT⿰ Antagonismus an 5-HT } \mathrm{H}_{2} \text {-Rezep- } \\
\text { toren, } a_{1} \text { Antagonismus an } \alpha_{1} \text {-Adrenozeptoren; }+++ \text { sehr stark, }++ \text { stark, }+ \text { schwach, } \\
+/- \text { sehr schwach, } 0 \text { nicht wirksam. } \\
\text { * Agomelatin: selektiver, spezifischer Agonismus am Melatoninrezeptor, Antagonismus an } \\
\text { 5-HT-Rezeptoren. }\end{array}$} \\
\hline
\end{tabular}

Mit freundlicher Genehmigung des Springer Nature Verlages 
Tabelle A 3 Häufigkeit relevanter unerwünschter Wirkungen von Antidepressiva in der klinischen Praxis. In Anlehnung an Regen und Benkert (2019), gekürzt auf Sedierung

\begin{tabular}{|c|c|}
\hline Antidepressivum & Sedierung \\
\hline Agomelatin & + \\
\hline Amitriptylin & +++ \\
\hline Amitriptylinoxid & +++ \\
\hline Bupropion & 0 \\
\hline Citalopram & 0 \\
\hline Clomipramin & + \\
\hline Doxepin & +++ \\
\hline Duloxetin & 0 \\
\hline Escitalopram & 0 \\
\hline Fluoxetin & 0 \\
\hline Fluvoxamin & $(+)$ \\
\hline Hypericum & + \\
\hline Imipramin & + \\
\hline Maprotilin & ++ \\
\hline Mianserin & ++ \\
\hline Milnacipran & 0 \\
\hline Mirtazapin & ++ \\
\hline Moclobemid & 0 \\
\hline Paroxetin & 0 \\
\hline Reboxetin & 0 \\
\hline Sertralin & 0 \\
\hline Tianeptin & 0 \\
\hline Tranylcypromin & 0 \\
\hline Trazodon & +++ \\
\hline Trimipramin & +++ \\
\hline Venlafaxin & 0 \\
\hline Vortioxetin & 0 \\
\hline
\end{tabular}

Mit freundlicher Genehmigung des Springer Nature Verlages 
Tabelle A 4 Rezeptorwirkungsprofile von Antipsychotika. In Anlehnung an Müller und Benkert (2019)

\begin{tabular}{|c|c|c|c|}
\hline Antipsychotikum & $\mathbf{H}_{1}$ & $5-\mathrm{HT}_{2}$ & $\alpha_{1}$ \\
\hline Amisulprid & 0 & 0 & 0 \\
\hline Aripiprazol & + & ++ & + \\
\hline Asenapin & + & ++ & + \\
\hline Benperidol & 0 & ++ & + \\
\hline Bromperidol & 0 & 0 & + \\
\hline Cariprazin & + & ++ & + \\
\hline Chlorprothixen & +++ & ++ & + \\
\hline Clozapin & +++ & +++ & + \\
\hline Flupentixol & + & ++ & + \\
\hline Fluphenazin & ++ & ++ & ++ \\
\hline Fluspirilen & 0 & + & 0 \\
\hline Haloperidol & 0 & + & ++ \\
\hline Levomepromazin & +++ & + & ++ \\
\hline Loxapin & +++ & +++ & +++ \\
\hline Lurasidon & 0 & +++ & ++ \\
\hline Melperon & + & ++ & + \\
\hline Olanzapin & +++ & +++ & ++ \\
\hline Paliperidone & + & +++ & + \\
\hline Perazin & +++ & ++ & ++ \\
\hline Perphenazin & ++ & ++ & ++ \\
\hline Pimozid & 0 & ++ & 0 \\
\hline Pipamperon & 0 & ++ & + \\
\hline Prothipendyl & $?$ & $?$ & $?$ \\
\hline Quetiapin & ++ & + & + \\
\hline Risperidon & + & +++ & +++ \\
\hline Sertindol & 0 & +++ & ++ \\
\hline Sulpirid & 0 & 0 & 0 \\
\hline Thioridazin & + & ++ & +++ \\
\hline Ziprasidon & ++ & +++ & + \\
\hline Zuclopenthixol & +++ & 0 & +++ \\
\hline
\end{tabular}

Mit freundlicher Genehmigung des Springer Nature Verlages 


\subsection{Vergleich von „Hypnotika“-Studien}

Für die vorliegende Arbeit wurde eine Liste von schlafanstoßenden Medikamenten - „Нypnotika“ - erstellt, die alle Wirkstoffe enthalten sollte, die potenziell in der Klinik eingesetzt werden könnten. Wie diese Liste erstellt wurde ist in Anhang 8.2 ausgeführt. Um einen Vergleich mit anderen Studien herzustellen und zu verhindern, dass Medikamente fälschlicherweise Medikamente als schlafanstoßende Mittel definiert werden, wurde eine Vergleichsliste von in der vorliegenden Dissertation erhobenen Wirkstoffen und anderer Studien über Schlafmittel erstellt (Tabelle A 5).

Studien, die sich auf „Hypnotika“ beziehen sind die von Eide und Schjøtt (2001), Opedal et al. (1998), Tom et al. (2016) und Glass et al. (2005). 
Tabelle A 5 Auswahl von sieben Studien, in denen schlafanstoßende Mittel ("Hypnotika") untersucht wurden. Ein "Ja" heißt, dass das Medikament in der Studie als Hypnotikum erhoben wurde. Medikamente in Zeilen ohne farbliche Füllung kamen in keiner der Studien vor. Farblich gekennzeichnete Zeilen zeigen, dass der Wirkstoff in mindestens einer anderen Studie erhoben wurde.

\begin{tabular}{|c|c|c|c|c|c|c|c|c|c|c|}
\hline $\mathbf{N r}$ & Wirkstoffgruppe & Wirkstoffname & $\begin{array}{l}\text { (Enzensper- } \\
\text { ger und } \\
\text { Lehmann } \\
\text { 2007) }\end{array}$ & $\begin{array}{c}\text { (Reeve } \\
\text { et al. } \\
2017 \text { ) }\end{array}$ & $\begin{array}{c}(\text { Tre- } \\
\text { win et } \\
\text { al. } \\
\text { 1992) }\end{array}$ & $\begin{array}{l}\text { (Eide } \\
\text { und } \\
\text { Schjøtt } \\
\text { 2001) }\end{array}$ & $\begin{array}{c}\text { (Ope- } \\
\text { dal et } \\
\text { al. } \\
\text { 1998) }\end{array}$ & $\begin{array}{l}\text { (Tom } \\
\text { et al. } \\
2016)\end{array}$ & $\begin{array}{c}\text { (Glass } \\
\text { et al. } \\
2005)^{*}\end{array}$ & $\begin{array}{c}\text { In We- } \\
\text { ende } \\
\text { verab- } \\
\text { reicht? }\end{array}$ \\
\hline 1 & Andere & Baldrian & & & & & & & & $\mathrm{Ja}$ \\
\hline 2 & Andere & Chloralhydrat & & & $\mathrm{Ja}$ & & $\mathrm{Ja}$ & $\mathrm{Ja}$ & & Nein \\
\hline 4 & Andere & Diphenhydramin & & & & & & & $\mathrm{Ja}$ & Nein \\
\hline 5 & Andere & Doxylamin & & & & & & & $\mathrm{Ja}^{* *}$ & Nein \\
\hline 6 & Andere & Melatonin & & & & & & & & $\mathrm{Ja}$ \\
\hline
\end{tabular}

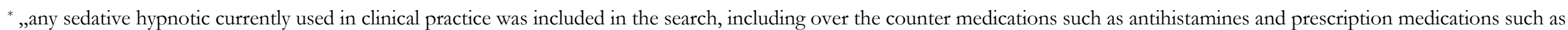
benzodiazepines and zolpidem, zopiclone, and zaleplon."

** „We searched Medline, Embase, the Cochrane clinical trials database, PubMed, and PsychLit from 1966 to 2003, using the Keywords [...] “antibistamines” [...] or “diphenhydramine [...].” 


\begin{tabular}{|c|c|c|c|c|c|c|c|c|c|c|}
\hline $\mathbf{N r}$ & Wirkstoffgruppe & Wirkstoffname & $\begin{array}{l}\text { (Enzensper- } \\
\text { ger und } \\
\text { Lehmann } \\
\text { 2007) }\end{array}$ & $\begin{array}{c}\text { Reeve } \\
\text { et al. } \\
2017 \text { ) }\end{array}$ & $\begin{array}{c}\text { (Tre- } \\
\text { win et } \\
\text { al. } \\
\text { 1992) }\end{array}$ & $\begin{array}{l}\text { (Eide } \\
\text { und } \\
\text { Schjøtt } \\
\text { 2001) }\end{array}$ & $\begin{array}{c}\text { (Ope- } \\
\text { dal et } \\
\text { al. } \\
\text { 1998) }\end{array}$ & $\begin{array}{l}\text { (Tom } \\
\text { et al. } \\
2016)\end{array}$ & $\begin{array}{l}\text { (Glass } \\
\text { et al. } \\
2005)^{*}\end{array}$ & $\begin{array}{c}\text { In We- } \\
\text { ende } \\
\text { verab- } \\
\text { reicht? }\end{array}$ \\
\hline 10 & Antidepressiva & Maprotilin & $\mathrm{Ja}$ & & & & & & & $\mathrm{Ja}$ \\
\hline 11 & Antidepressiva & Mianserin & $\mathrm{Ja}$ & & & $\mathrm{Ja}$ & $\mathrm{Ja}$ & & & $\mathrm{Ja}$ \\
\hline 12 & Antidepressiva & Mirtazapin & $\mathrm{Ja}$ & & & & & $\mathrm{Ja}$ & & $\mathrm{Ja}$ \\
\hline 13 & Antidepressiva & Opipramol & & & & & & & & $\mathrm{Ja}$ \\
\hline 14 & Antidepressiva & Trazodon & $\mathrm{Ja}$ & & & & & $\mathrm{Ja}$ & & $\mathrm{Ja}$ \\
\hline 15 & Antidepressiva & Trimipramin & $\mathrm{Ja}$ & & & & & & & $\mathrm{Ja}$ \\
\hline 16 & Benzodiazepine - kurz u. mittellang & Alprazolam & & $\mathrm{Ja}$ & $\mathrm{Ja}$ & & & & & Nein \\
\hline 17 & Benzodiazepine - kurz u. mittellang & Brotizolam & & & & & & & & Nein \\
\hline 18 & Benzodiazepine - kurz u. mittellang & Lorazepam & & $\mathrm{Ja}$ & $\mathrm{Ja}$ & & & $\mathrm{Ja}$ & & $\mathrm{Ja}$ \\
\hline 19 & Benzodiazepine - kurz u. mittellang & Lormetazepam & & $\mathrm{Ja}^{* * *}$ & $\mathrm{Ja}$ & & & & & $\mathrm{Ja}$ \\
\hline 20 & Benzodiazepine - kurz u. mittellang & Nitrazepam & & $\mathrm{Ja}$ & $\mathrm{Ja}$ & $\mathrm{Ja}$ & $\mathrm{Ja}$ & & & $\mathrm{Ja}$ \\
\hline 21 & Benzodiazepine - kurz u. mittellang & Oxazepam & & $\mathrm{Ja}$ & & $\mathrm{Ja}$ & $\mathrm{Ja}$ & $\mathrm{Ja}$ & & $\mathrm{Ja}$ \\
\hline 22 & Benzodiazepine - kurz u. mittellang & Temazepam & & $\mathrm{Ja}$ & $\mathrm{Ja}$ & & & $\mathrm{Ja}$ & & $\mathrm{Ja}$ \\
\hline
\end{tabular}

${ }^{* * *}$ Lormetazepam wurde als temporäre Substitution der regulären BZD Therapie genutzt 


\begin{tabular}{|c|c|c|c|c|c|c|c|c|c|c|}
\hline $\mathbf{N r}$ & Wirkstoffgruppe & Wirkstoffname & $\begin{array}{l}\text { (Enzensper- } \\
\text { ger und } \\
\text { Lehmann } \\
\text { 2007) }\end{array}$ & $\begin{array}{c}\text { Reeve } \\
\text { et al. } \\
2017 \text { ) }\end{array}$ & $\begin{array}{c}\text { (Tre- } \\
\text { win et } \\
\text { al. } \\
\text { 1992) }\end{array}$ & $\begin{array}{l}\text { (Eide } \\
\text { und } \\
\text { Schjøtt } \\
\text { 2001) }\end{array}$ & $\begin{array}{c}\text { (Ope- } \\
\text { dal et } \\
\text { al. } \\
\text { 1998) }\end{array}$ & $\begin{array}{l}\text { (Tom } \\
\text { et al. } \\
\text { 2016) }\end{array}$ & $\begin{array}{l}\text { (Glass } \\
\text { et al. } \\
\text { 2005)* }\end{array}$ & $\begin{array}{l}\text { In We- } \\
\text { ende } \\
\text { verab- } \\
\text { reicht? }\end{array}$ \\
\hline 23 & Benzodiazepine - kurz u. mittellang & Tetrazepam & & & & & & & & $\mathrm{Ja}$ \\
\hline 24 & Benzodiazepine - kurz u. mittellang & Triazolam & & $\mathrm{Ja}$ & $\mathrm{Ja}$ & & & $\mathrm{Ja}$ & & Nein \\
\hline 25 & Benzodiazepine - Lang & Bromazepam & & $\mathrm{Ja}$ & & & & & & $\mathrm{Ja}$ \\
\hline 26 & Benzodiazepine - Lang & Chlordiazepoxid & & $\mathrm{Ja}$ & $\mathrm{Ja}$ & & & & & Nein \\
\hline 27 & Benzodiazepine - Lang & Clobazam & & $\mathrm{Ja}$ & & & & & & Nein \\
\hline 28 & Benzodiazepine - Lang & Diazepam & & $\mathrm{Ja}$ & $\mathrm{Ja}$ & $\mathrm{Ja}$ & $\mathrm{Ja}$ & & & $\mathrm{Ja}$ \\
\hline 29 & Benzodiazepine - Lang & Dikaliumclorazepat & & $\mathrm{Ja}$ & $\mathrm{Ja}$ & & & & & $\mathrm{Ja}$ \\
\hline 30 & Benzodiazepine - Lang & Flunitrazepam & & $\mathrm{Ja}$ & $\mathrm{Ja}$ & $\mathrm{Ja}$ & $\mathrm{Ja}$ & & & $\mathrm{Ja}$ \\
\hline 31 & Benzodiazepine - Lang & Flurazepam & & $\mathrm{Ja}$ & $\mathrm{Ja}$ & & & $\mathrm{Ja}$ & & $\mathrm{Ja}$ \\
\hline 32 & Benzodiazepine - Lang & Medazepam & & $\mathrm{Ja}$ & & & & & & Nein \\
\hline 33 & Benzodiazepine - Lang & Prazepam & & $\mathrm{Ja}$ & & & & & & Nein \\
\hline 34 & Neuroleptika & Aripiprazol & & & & & & & & Nein \\
\hline 35 & Neuroleptika & Asenapin & & & & & & & & Nein \\
\hline 36 & Neuroleptika & Benperidol & & & & & & & & $\mathrm{Ja}$ \\
\hline
\end{tabular}




\begin{tabular}{|c|c|c|c|c|c|c|c|c|c|c|}
\hline $\mathrm{Nr}$ & Wirkstoffgruppe & Wirkstoffname & $\begin{array}{l}\text { (Enzensper- } \\
\text { ger und } \\
\text { Lehmann } \\
\text { 2007) }\end{array}$ & $\begin{array}{c}\text { (Reeve } \\
\text { et al. } \\
2017 \text { ) }\end{array}$ & $\begin{array}{c}(\text { Tre- } \\
\text { win et } \\
\text { al. } \\
1992)\end{array}$ & $\begin{array}{l}\text { (Eide } \\
\text { und } \\
\text { Schjøtt } \\
\text { 2001) }\end{array}$ & $\begin{array}{c}\text { (Ope- } \\
\text { dal et } \\
\text { al. } \\
\text { 1998) }\end{array}$ & $\begin{array}{l}\text { (Tom } \\
\text { et al. } \\
2016 \text { ) }\end{array}$ & $\begin{array}{l}\text { (Glass } \\
\text { et al. } \\
2005)^{*}\end{array}$ & $\begin{array}{l}\text { In We- } \\
\text { ende } \\
\text { verab- } \\
\text { reicht? }\end{array}$ \\
\hline 37 & Neuroleptika & Chlorprothixen & $\mathrm{Ja}$ & & & & & & & Nein \\
\hline 38 & Neuroleptika & Clozapin & & & & & & & & Nein \\
\hline 40 & Neuroleptika & Fluphenazin & & & & & & & & Nein \\
\hline 41 & Neuroleptika & Haloperidol & & & & & & & & $\mathrm{Ja}$ \\
\hline 42 & Neuroleptika & Levomepromazin & $\mathrm{Ja}$ & & & $\mathrm{Ja}$ & $\mathrm{Ja}$ & & & Nein \\
\hline 46 & Neuroleptika & Perphenazin & & & & & & & & Nein \\
\hline 47 & Neuroleptika & Pipamperon & $\mathrm{Ja}$ & & & & & & & $\mathrm{Ja}$ \\
\hline 48 & Neuroleptika & Promethazin & $\mathrm{Ja}$ & & & $\mathrm{Ja}$ & $\mathrm{Ja}$ & & & $\mathrm{Ja}$ \\
\hline 49 & Neuroleptika & Prothipendyl & $\mathrm{Ja}$ & & & & & & & $\mathrm{Ja}$ \\
\hline 50 & Neuroleptika & Quetiapin & $\mathrm{Ja}$ & & & & & & & $\mathrm{Ja}$ \\
\hline
\end{tabular}




\begin{tabular}{|c|c|c|c|c|c|c|c|c|c|c|}
\hline $\mathbf{N r}$ & Wirkstoffgruppe & Wirkstoffname & $\begin{array}{l}\text { (Enzensper- } \\
\text { ger und } \\
\text { Lehmann } \\
\text { 2007) }\end{array}$ & $\begin{array}{l}\text { Reeve } \\
\text { et al. } \\
2017 \text { ) }\end{array}$ & $\begin{array}{c}\text { (Tre- } \\
\text { win et } \\
\text { al. } \\
\text { 1992) }\end{array}$ & $\begin{array}{l}\text { (Eide } \\
\text { und } \\
\text { Schjøtt } \\
\text { 2001) }\end{array}$ & $\begin{array}{c}\text { (Ope- } \\
\text { dal et } \\
\text { al. } \\
\text { 1998) }\end{array}$ & $\begin{array}{l}\text { (Tom } \\
\text { et al. } \\
\text { 2016) }\end{array}$ & $\begin{array}{l}\text { (Glass } \\
\text { et al. } \\
\text { 2005)* }\end{array}$ & $\begin{array}{c}\text { In We- } \\
\text { ende } \\
\text { verab- } \\
\text { reicht? }\end{array}$ \\
\hline 51 & Neuroleptika & Risperidon & $\mathrm{Ja}$ & & & & & & & $\mathrm{Ja}$ \\
\hline 52 & Neuroleptika & Thioridazin & $\mathrm{Ja}$ & & & & & & & Nein \\
\hline 53 & Neuroleptika & Zuclopenthixol & & & & & & & & Nein \\
\hline 54 & OP-Medikation & Midazolam & & & & & & & & $\mathrm{Ja}$ \\
\hline 55 & Z-Substanzen & Zaleplon & & $\mathrm{Ja}$ & & & & $\mathrm{Ja}$ & $\mathrm{Ja}$ & Nein \\
\hline 56 & Z-Substanzen & Zolpidem & & $\mathrm{Ja}$ & & $\mathrm{Ja}$ & & $\mathrm{Ja}$ & $\mathrm{Ja}$ & $\mathrm{Ja}$ \\
\hline 57 & Z-Substanzen & Zopiclon & & $\mathrm{Ja}$ & & $\mathrm{Ja}$ & $\mathrm{Ja}$ & & $\mathrm{Ja}$ & $\mathrm{Ja}$ \\
\hline
\end{tabular}




\subsection{Standard Operating Procedure}

Auf den folgenden Seiten findet sich die SOP (s.a. Kapitel 4.6.2.2).

Die SOP stellt eine Anleitung für die Datenabstraktoren dar, in der jeder Punkt der Dateneingabemaske erklärt wird. Zu jedem Punkt wird erläutert, wie Informationen dazu in der Patientenakte zu finden sind. Zusätzlich wurden Entscheidungen festgehalten, wie bestimmte Angaben aus den Akten in die Eingabemaske übertragen wurden. 


\section{Inhaltsverzeichnis}

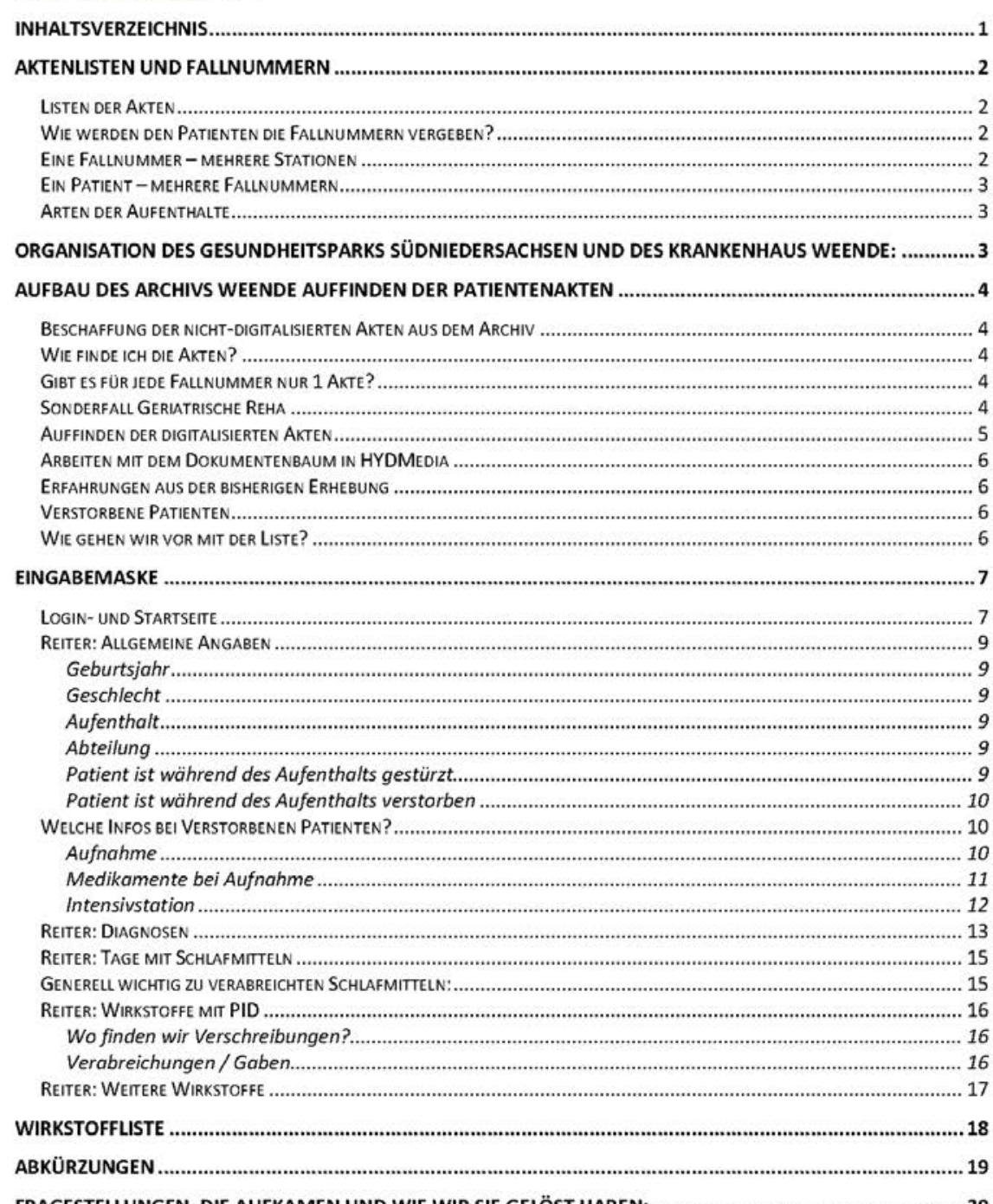




\section{Aktenlisten und Fallnummern}

\section{Listen der Akten}

Es gibt 2 Listen von Fallnummern, die für uns erstellt wurden.

1. Liste: 01.07 .2013 bis 31.12 .2013

2. Liste: 01.07 .2017 bis 31.12 .2017

Die Listen führen alle Fallnummern mit Patienten, die 65 Jahre oder älter sind, auf. Die Fallnummern sind nach Aufnahmedatum geordnet (Abbildung 1).

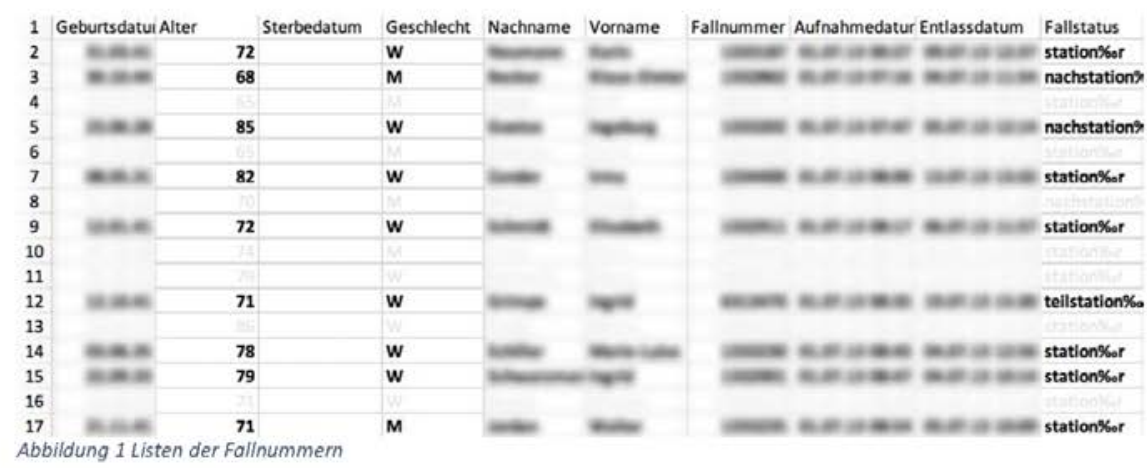

Schwarz und Fett: alle Fälle, die im Krankenhaus Weende stattgefunden haben und in unserer Studie sind.

: alle Fälle, die an den Standorten Neu-Mariahilf oder Lenglern stattgefunden haben und nicht relevant für unsere Erhebung sind.

Die Liste 2013 wollen wir Fallnummer für Fallnummer in die Datenbank aufnehmen, bis wir 1000 Einträge in unserer Datenbank haben.

Die Liste 2017 wollen wir Fallnummer für Fallnummer in die Datenbank aufnehmen, für den Zeitraum, der durch die Erhebung der ersten 1000 Fälle der Liste 2013 festgelegt wurde. Update (nach der Erhebung 2013): Der Zeitraum für 2017 ist festgelegt für alle Aufnahmen 01.07.2017 - 11.08.2017

Wie werden den Patienten die Fallnummern vergeben?

Eine Fallnummer wird einem Patienten vergeben, wenn er im Krankenhaus aufgenommen wird. Diese Nummer kann bei Verlegung auf eine andere Station gleichbleiben - es kann auch sein, dass für den weiteren Aufenthalt eine neue Fallnummer vergeben wird.

Eine Fallnummer - mehrere Stationen

Es kann sein, dass ein Patient unter einer Fallnummer auf mehreren verschiedenen Stationen behandelt wird.

Beispiel:

Ein Patient wird in der Allgemeinchirurgie (ACH) operiert, danach für die Nachversorgung auf eine Station der Inneren Medizin (IM) verlegt und dann in die ambulante Weiterversorgung entlassen. 
Der Patient hat beispielsweise die Fallnummer 3346222 für den Aufenthalt auf der Allgemeinchirurgie, wo der Patient für eine Operation behandelt wird. Dann kommt der Patient nach der Operation auf eine Station der Inneren Medizin, wo der Aufenthalt weiter mit der Fallnummer 3364222 geführt wird.

Für den Aufenthalt auf der Station der Allgemeinchirurgie wird ein Arztbrief geschrieben, um den Patienten auf die neue Station zu verschieben. Für den darauffolgenden Aufenthalt auf der Station der Inneren Medizin wird auch ein Arztbrief geschrieben um den Patienten dann in die ambulante Versorgung zu entlassen.

Für uns sind diese beiden Aufenthalte $(A C H+I M) 2$ unterschiedliche Fälle.

Wir erstellen also für den ersten Aufenthalt in der $\mathrm{ACH}$ einen Fall in unserer Eingabemaske und für den Aufenthalt in der IM einen neuen Fall in unserer Eingabemaske (Tabelle 1).

Tabelle 1 Eine Fallnummer - mehrere Stationen

\begin{tabular}{|l|l|l|}
\hline Fallnummer & Station & Fall in unserer Studie \\
\hline 3346222 & Allgemeinchirurgie (ACH) & Fall 1 \\
\hline 3346222 & Innere Medizin (IM) & Fall 2 \\
\hline
\end{tabular}

In dem Programm ORBIS kann es sein, dass dieser Fall nur als ein Aufenthalt auf einer der beiden Stationen eingeordnet ist.

Ein Patient - mehrere Fallnummern

Wird ein Patient entlassen und daraufhin wieder aufgenommen, erhält er oft eine neue Fallnummer. Deswegen kann es sein, dass wir denselben Patienten mehrere Male in unsere Datenbank aufnehmen werden.

Wird ein Patient von einer Abteilung auf eine andere Abteilung verlegt, kann es sein, dass er dort eine neue Fallnummer bekommt.

Da unsere Liste alle Fallnummern in chronologischer Folge auflistet, die wir erheben wollen, müssen wir die neue Fallnummer nur erheben, wenn sie auch auf der Liste ist.

Wir arbeiten die Liste chronologisch ab und damit sollte diese neue Fallnummer - weiter unten - auch auf der Liste sein.

Wurde ein Patient in die Datenbank aufgenommen, muss der Fall auf der Liste gekennzeichnet werden.

\section{Arten der Aufenthalte}

Wir wollen stationäre Aufenthalte erheben, bei denen die Patienten ihre Nächte im Krankenhaus verbringen.

Unter den Fallnummern sind auch Aufenthalte in der Geriatrischen Tagesklinik (GTK). Diese Fälle wollen wir nicht mit aufnehmen.

\section{Standorte des Krankenhaus Weende}

Das Evangelische Krankenhaus Göttingen-Weende hat 3 Standorte:

- Göttingen-Weende

- Bovenden-Lenglern

- Göttingen/Neu-Mariahilf 
Wir nehmen nur Fälle und Akten aus dem Standort Göttingen-Weende auf.

\section{Aufbau des Archivs Weende Auffinden der Patientenakten \\ Beschaffung der nicht-digitalisierten Akten aus dem Archiv \\ Die Akten sind im Archiv gelagert.}

Jeder Fallnummer ist normalerweise 1 Papierakte zugeordnet. Es kann auch sein, dass es für eine Fallnummer mehrere Akten gibt, wenn der Aufenthalt lang ist.

Die Akten werden jeden Beginn des Arbeitstags aus dem Archiv geholt und müssen am Ende des Arbeitstags wieder in das Archiv gebracht werden. Die Akten, die wir am Anfang des Tages aus dem Archiv mitnehmen, müssen wir auf einer Extra-Liste, die wir unten im Archiv lassen, vermerken.

Hier kennzeichnen wir die Fallnummern mit " $x$ " und zwar folgendermaßen:

$x$ : Haben wir mitgenommen

xx: Haben wir fertig bearbeitet

xxx: Sind wieder in den Schrank eingeordnet

Auf diese Weise hat auch das Personal des Archivs einen Überblick über unsere Arbeit.

Wie finde ich die Akten?

Die Akten sind nach Behandlungsjahr und Fallnummer in den Schränken des Archivs geordnet.

Gibt es für jede Fallnummer nur 1 Akte?

Bei längeren Aufenthalten kann es passieren, dass die Patienten viel Dokumentation und dementsprechend viel Papier brauchen. In solchen Fällen können für eine Fallnummer (1 Aufenthalt) mehrere Akten vorhanden sein. Die Akten sind dann im Archiv nebeneinander am gleichen Ort aufzufinden.

Sonderfall Geriatrische Reha

Es kann vorkommen, dass ein Patient nach einem stationären Aufenthalt in zum Beispiel der Inneren Medizin aufgrund eines akuten Ereignisses auf eine geriatrische Rehabilitationsstation kommt. In dem Fall sind die Akten mit der Fallnummer für den ersten akuten Aufenthalt den Akten aus der geriatrischen Rehabilitation zugeordnet und in den Schränken, wo die geriatrischen Rehabilitationskurven aufbewahrt sind, unter der geriatrischen Fallnummer, aufzufinden.

Beispiel: 
Aufenthalt 1 auf einer Station der Inneren Medizin:

1337092

Aufenthalt 2 auf einer Station der geriatrischen Rehabilitation:

Dokumente zu Aufenthalt 1 und Aufenthalt 2 sind in der Akte des Aufenthalts 2 der geriatrischen Rehabilitation zu finden.

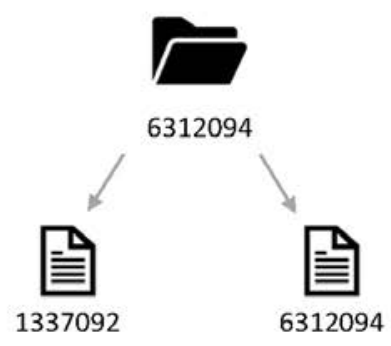

Auffinden der digitalisierten Akten

1. Schritt: „Orbis OneKis"

- Einloggen in den Orbis-Account

- Fallnummer eingeben, um den Patienten zu finden

- In dem Fenster, in dem die Aufenthalte gelistet sind, den gesuchten Aufenthalt raussuchen -> Rechtsklick -> "digitales Archiv“ anklicken

- Es öffnet sich das Programm "HYDMedia G5"

2. „HYDMedia"

- In der linken Leiste „Dokumentenbaum“ finden sich alle digitalisierten Aufenthalte des Patienten.

- Auswahl des gesuchten Aufenthalts durch Anklicken

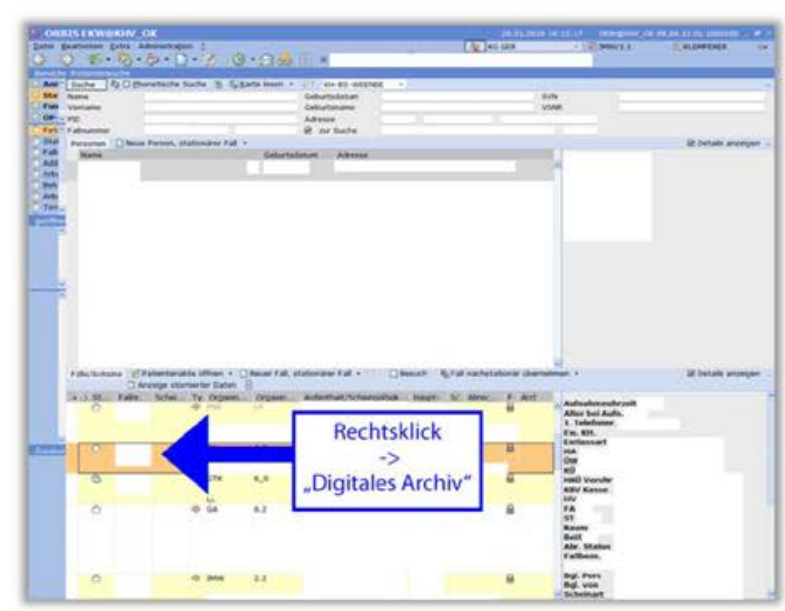

Abbildung 2 Zugriff auf die digitale Akte von Orbis 


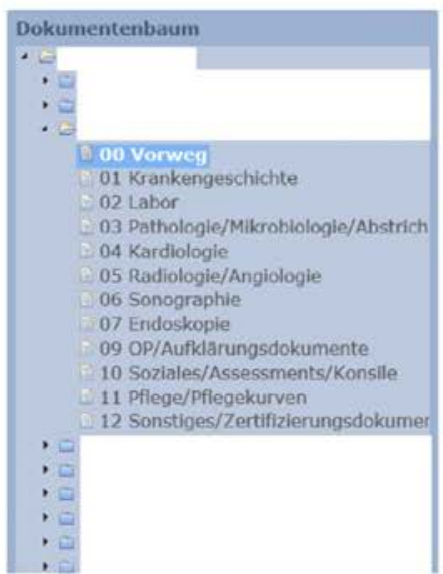

Abbildung 3 HYDMedia Dokumentenbaum
Arbeiten mit dem Dokumentenbaum in HYDMedia Die gescannten Akten sind in den Reitern "00 Vorweg" bis "12 Sonstiges/Zertifizierungsdokumente" geordnet. Erfahrungen aus der bisherigen Erhebung (Stand: 12.02.19):

\begin{tabular}{|c|c|}
\hline 00 & $\begin{array}{l}\text { - Arztbriefe } \\
\begin{aligned} & \circ \text { für den Aufenthalt } \\
& \text { aus vorherigen Aufenthalten } \\
& \text { Krankenhaus-interne Arztbriefe } \\
& \text { (z.B. IMC-Briefe) }\end{aligned}\end{array}$ \\
\hline 01 & $\begin{array}{l}\text { - Aufnahmedokumente } \\
\text { - Medikamentenpläne } \\
\text { - Unfallprotokolle }\end{array}$ \\
\hline$\ldots$ & $\ldots$ \\
\hline 09 & - Anästhesieprotokoll \\
\hline 10 & - Neuropsychologie-Befunde \\
\hline 11 & $\begin{array}{l}\text { - Pflegedokumente } \\
\text { - Intensivstationsaufenthalte }\end{array}$ \\
\hline 12 & $\begin{array}{l}\text { - "Unfallprotokoll“ (kann ein } \\
\text { Sturzprotokoll sein) }\end{array}$ \\
\hline
\end{tabular}

Die Reiter „02 Labor" bis „07 Endoskopie“ enthalten keine Dokumente, die für uns primär wichtig sind. ${ }^{1}$ Es kann aber helfen die Dokumente anzugucken, um Unklarheiten in einem Aufenthalt zu verstehen.

\section{Verstorbene Patienten}

Wenn ein Patient während dem Aufenthalt im Krankenhaus verstorben ist, ist es wichtig das Feld „Patient ist während des Aufenthalts verstorben“ mit „Ja" zu kennzeichnen.

Die restlichen Informationen, die wir über diesen Patienten aufnehmen sind nur wenige: Im Reiter "Allgemeine Angaben“:

- Geburtsjahr

- Krankenhaus

- Aufenthalt

- Geschlecht

- Abteilung

Danach kann der Fall abgeschlossen werden.

Wie gehen wir vor mit der Liste?

Wir arbeiten die Liste chronologisch ab.

\footnotetext{
${ }^{1}$ Wissensstand nach Rücksprache mit dem Archiv (Dezember 2018) und mit Simone Assmann (22.01.19)
} 
JONAS KLEMPERER

\section{STANDARD OPERATING PROCEDURE - WEENDE}

Jeder von uns hat eine eigene Liste, an der er/sie sich orientieren kann um die Fälle einzutragen. Wenn ein Fall in die Eingabemaske eingetragen wurde, vermerken wir das auf unserer Liste.

Eingabemaske

https://www.allgemeinmedizin2.med.uni-goettingen.de/intervent/

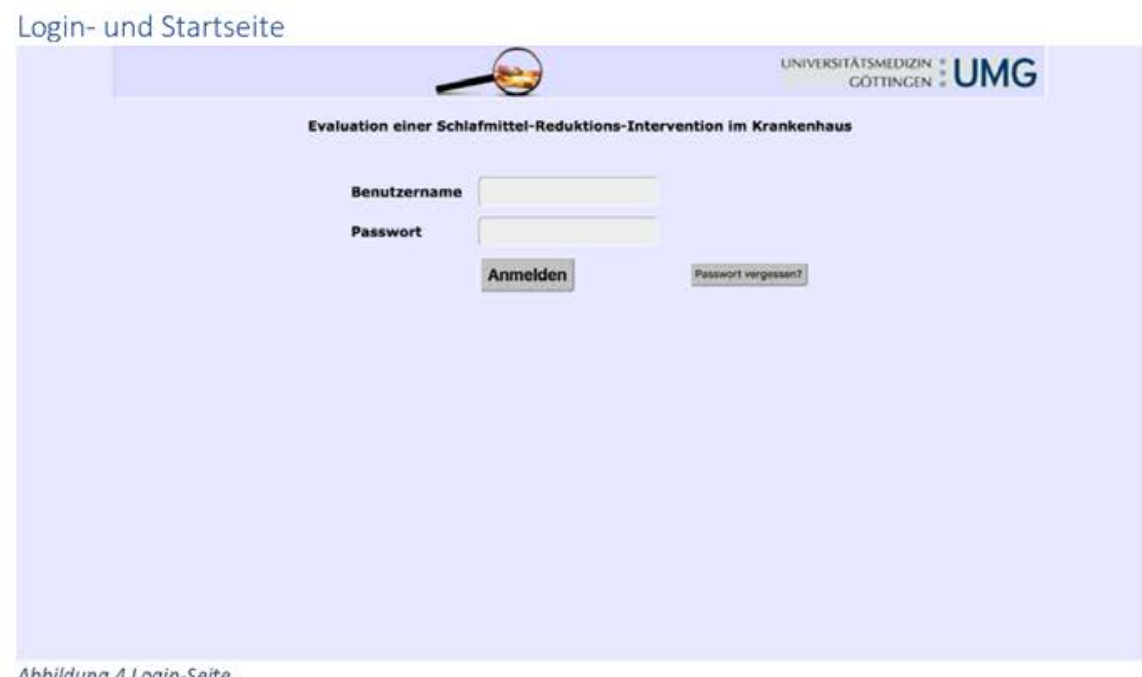

Auf der Login-Seite (Abbildung 4) können wir uns mit unserem Benutzernamen und Passwort anmelden. 
JONAS KLEMPERER

STANDARD OPERATING PROCEDURE - WEENDE

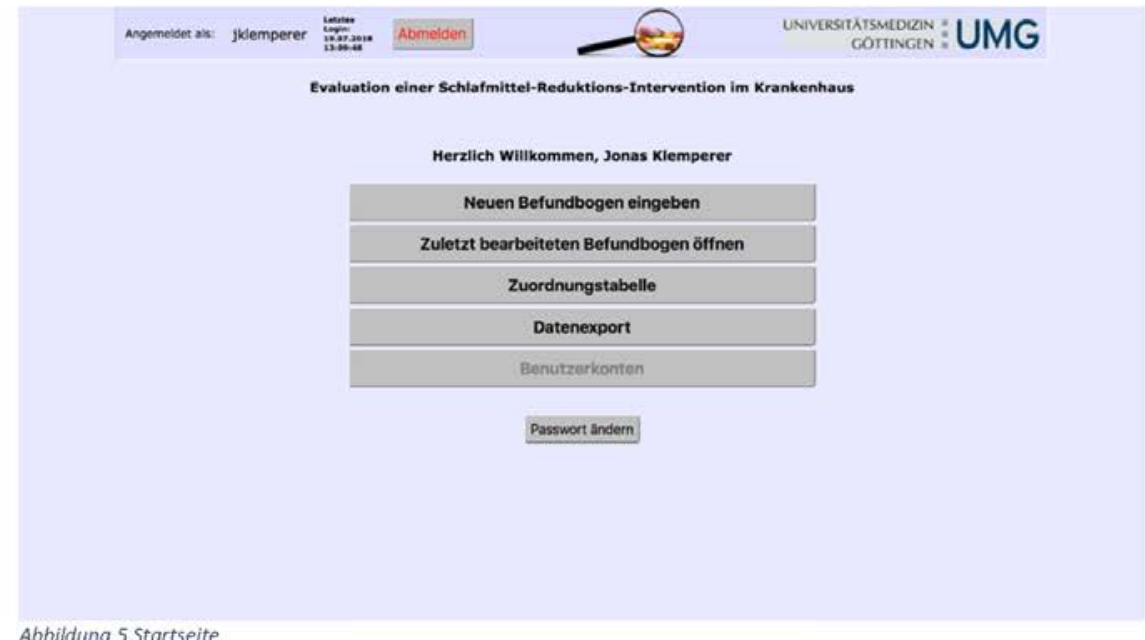

Abbildung 5 Startseite

Nach der Anmeldung mit Benutzernamen und Passwort öffnet sich die Startseite (Abbildung 5)

Neuen Befundbogen eingeben: Hiermit können wir einen neuen Eintrag in unserer Datenbank machen. Bei der Auswahl dieses Feldes wird ein Eintrag erstellt und in der Bearbeitungsansicht geöffnet.

Zuletzt bearbeiteten Befundbogen öffnen: Die Auswahl dieses Feldes bringt den Benutzer zu dem zuletzt geöffneten Befundbogen zurück.

Zuordnungstabelle: Bei Auswahl dieses Feldes öffnet sich die Tabelle mit allen bisher erstellten Befundbögen. Diese können bearbeitet, beschriftet und gelöscht werden. 
Reiter: Allgemeine Angaben

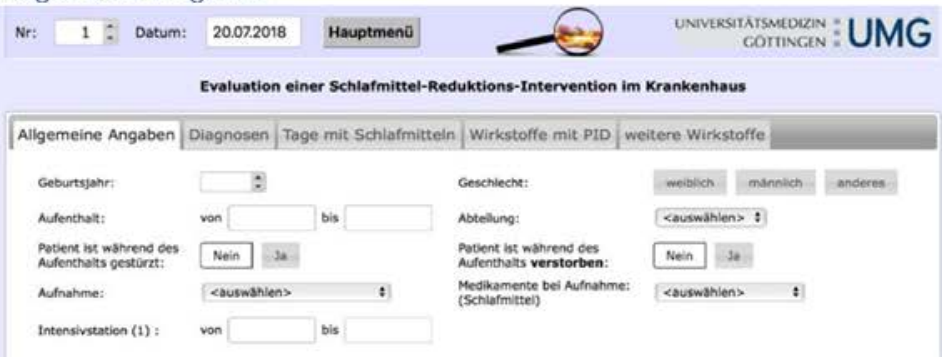

Abbildung 6 Allgemeine Angaben

Auszufüllende Felder:

Geburtsjahr: Das Geburtsjahr des Patienten

Geschlecht: Das Geschlecht des Patienten

Aufenthalt: Datum des ersten Tags des Aufenthalts und Datum des Tags der Entlassung.

Abteilung: In welcher Abteilung hat der Aufenthalt stattgefunden?

Auswahlmöglichkeiten:

- Innere
- Chirurgie
- Geriatrie

\begin{tabular}{|c|c|}
\hline Organisationseinheit & Abteilung \\
\hline IM & Innere \\
IMW & Chirurgie \\
\hline ACH & \\
GYN & \\
PCH & \\
UCH & \\
UR & Geriatrie \\
\hline GA & \\
\hline GR & \\
\hline
\end{tabular}

Schmerztherapie: In unserer Patientenliste sind Patienten, die einen stationären Aufenthalt über die Schmertherapie haben. Wir nehmen diese Patienten auf, ordnen sie unter "Innere“ ein und müssen sie (wichtig!) in der Zuordnungstabelle mit "Schmerztherapie" kennzeichnen.

Patient ist während des Aufenthalts gestürzt: „Ja“, wenn in der Akte ein Sturzprotokoll aufzufinden ist für einen Sturz, der während dem Zeitraum des Aufenthalts stattgefunden hat. 
Patient ist während des Aufenthalts verstorben: „Ja“, wenn in der Akte eine Todesbescheinigung aufzufinden ist, für das Versterben des Patienten während des Zeitraums. Auch ein „Exitus letalis“ im Arztbrief für den Zeitraum kann als Tod bewertet werden.

\section{Aufnahme: Aus welchem} Versorgungskontext kommt ein Patient für den Aufenthalt? Auswahlmöglichkeiten:

- von zu Hause

- aus Pflegeheim

- aus anderem Krankenhaus

- aus anderer Abteilung

- unbekannt

Die Aufnahme wird in Arztbriefen nicht standardisiert beschrieben. Also müssen wir mehrere Informationen nutzen, um zu einer Aussage zu kommen.

Was wir hierbei beachten können um eine möglichst verlässliche Aussage zu treffen:

\section{Welche Infos bei Verstorbenen}

Patienten?

Wenn ein Patient während dem Aufenthalt im Krankenhaus verstorben ist, ist es wichtig das Feld „Patient ist während des Aufenthalts verstorben “ mit „Ja" zu kennzeichnen.

Die restlichen Informationen, die wir über diesen Patienten aufnehmen sind nur wenige:

Im Reiter „Allgemeine Angaben“:

- Geburtsjahr

- Krankenhaus

- Aufenthalt

- Geschlecht

- Abteilung

Danach kann der Fall abgeschlossen werden.

- Arztbriefe von direkt vorher anschließenden Aufenthalten

- Von anderen Stationen des gleichen Hauses

- Von anderen Krankenhäusern

- Stammblatt (von der Pflege erhoben):

- Lebenssituation: ledig, verheiratet, verwitwet)

- Wohnsituation (woher): lebt mit Familie, lebt allein, Altenwohnanlage, Pflegeheim, Sozialstation/Pflegedienst, Fremdkrankenhaus, MRSA Risikopatient, Pflege-Überleitung

- Überleitbogen aus einem Pflegeheim

Ein paar Beispiele für Informationen, die zu den jeweiligen Aufnahme-Möglichkeiten passen:

\begin{tabular}{|l|l|}
\hline Aufnahme & Informationen aus der Akte \\
\hline von zu Hause & $\begin{array}{l}\text { Arztbrief beschreibt, dass zum Beispiel ein Unfall bei } \\
\text { der Gartenarbeit zuhause passiert ist und deswegen } \\
\text { die Aufnahme erfolgt. } \\
\text { - }\end{array}$ \\
\hline aus Pozialstation / Pflegedienst \\
\hline - Aus anderem Krankenhaus & $\begin{array}{l}\text { Arztbrief beschreibt, dass der Patient aus einem } \\
\text { Pflegeheim kommt. }\end{array}$ \\
& $\begin{array}{l}\text { Es ist ein Überleitbogen aus dem Pflegeheim } \\
\text { vorhanden. }\end{array}$ \\
\hline - Arztbrief beschreibt, dass der Patient von einem \\
anderen Krankenhaus aufgenommen wurde \\
- Es ist ein Arztbrief des anderen Krankenhauses \\
vorhanden, der zeitlich direkt (kein Tag Pause) an \\
den Aufenthalt im Krankenhaus Weende anschließt.
\end{tabular}




\begin{tabular}{|c|c|}
\hline & $\begin{array}{l}\text { Es ist in einem Arztbrief von einem vorherigen } \\
\text { Aufenthalt klar formuliert, dass der Patient zur } \\
\text { Weiterbehandlung ins EKW kommt. } \\
\text { - Aufnahme aus Lenglern/anderen Standorten des } \\
\text { Gesundheitsparks Südniedersachsen. }\end{array}$ \\
\hline aus anderer Abteilung & $\begin{array}{l}\text { - Im Arztbrief wird erwähnt, dass der Patient von einer } \\
\text { anderen Abteilung des KHW aufgenommen wird. } \\
\text { - Es ist ein Arztbrief über einen Aufenthalt im EKW } \\
\text { vorhanden, der direkt (kein Tag Pause) vor dem } \\
\text { jetzigen Aufenthalt stattgefunden hat. } \\
\text { - Es sind mehrere verschiedene Pflegekurven } \\
\text { vorhanden, die den Aufenthalt von mehreren } \\
\text { Stationen zeigen }\end{array}$ \\
\hline Unbekannt & $\begin{array}{l}\text { - Es sind keine/nicht ausreichend Informationen } \\
\text { vorhanden, um eine verlässliche Aussage zu treffen. }\end{array}$ \\
\hline
\end{tabular}

In der Bearbeitung der Fälle gefundene Formulierungen und wie wir sie gewertet haben:

\begin{tabular}{|l|l|}
\hline Aufnahme & Formulierung in der Akte \\
\hline Pflegeheim & - Betreutes Wohnen \\
& - Altenwohnanlage \\
- Wohnpark, Zimmermannstraße (Göttingen) \\
- Senioren-Park Carpe Diem (Göttingen) \\
- GDA-Wohnstift (Göttingen) \\
\hline
\end{tabular}

Was geben wir ein, wenn ein Patient nach Einlieferung in ein anderes Krankenhaus direkt in unser Krankenhaus weiterverlegt wurde?

Wenn der Patient keine Nacht in dem Krankenaus verbracht hat, von dem er kommt, zählen wir den Ort der Aufnahme, von dem der Patient vorher kam.

Beispiel: Ein Patient wird von zuhause in die Notaufnahme aufgenommen, dann am gleichen Tag weiter ins EKW: Aufnahme von zuhause.

Was geben wir ein, wenn ein Patient im (selben oder anderen) Krankenhaus lag, an dem Tag entlassen wurde und wieder aufgenommen wurde?

Wenn ein Patient direkt anschließend einen Aufenthalt in dem Krankenhaus Weende hatte oder einem anderen Krankenhaus - und Tag der Entlassung wieder aufgenommen wird, geben wir ein, dass der Patient "aus anderer Abteilung" oder "aus anderem Krankenhaus" aufgenommen wurde. (siehe Tabelle oben: Aufnahme „andere Abteilung“)

Medikamente bei Aufnahme:

Die Medikamente (nur Schlafmittel/Hypnotika), die dem Patienten zum Zeitpunkt der Aufnahme verschrieben waren (aus dem vorherigen Kontext):
- Nein
- Dauermedikation
- Bedarfsmedikation 


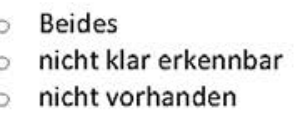

Welche Informationen können wir hier benutzen, um eine Auswahl zu treffen?

- Ärztlicher Anamnesebogen: „Vormedikation“

- Aktueller Medikamentenplan eines Patienten, der vom Hausarzt erstellt wurde.

- Aktueller Medikamentenplan eines Patienten, der vom Pflegeheim erstellt wurde (dann oft im Überleitbogen)

- Aktuelle Medikamente bei Aufnahme von einer anderen Station: In den ärztlichen Verordnungen für den Aufenthalt der direkt vorher anschließend war.

- Aktuelle Medikamente bei Aufnahme aus einem anderen Krankenhaus: Medikamente aus dem Arztbrief des direkt vorher anschließenden Aufenthalts.

\begin{tabular}{|l|l|}
\hline Auswahlmöglichkeit & $\begin{array}{l}\text { Medikamente (Verschreibung) bei } \\
\text { Aufnahme }\end{array}$ \\
\hline Nein & $\begin{array}{l}\text { kein Schlafmittel } \\
\text { ein oder mehrere Schlafmittel als } \\
\text { Dauermedikation }\end{array}$ \\
\hline Dauermedikation & $\begin{array}{l}\text { ein oder mehrere Schlafmittel als } \\
\text { Bedarfsmedikation }\end{array}$ \\
\hline Bedarfsmedikation & $\begin{array}{l}\text { Ein oder mehrere Schlafmittel als Dauer- } \\
\text { und Bedarfsmedikation. }\end{array}$ \\
\hline Beides & Die Schrift ist nicht zu lesen \\
\hline Nicht klar erkennbar & Die Medikation ist nicht aufzufinden. \\
\hline Nicht vorhanden & \\
\hline
\end{tabular}

Intensivstation

Aufenthalt eines Patienten auf der Intermediate Care Station (IMC) oder der Intensivstation (IS/ITS/IST). Wenn ein Aufenthalt von der IS direkt auf die IMC geht, kann man den Aufenthalt als einen Zeitraum angeben.

Beispiel:

04.07.-06.07. IS

06.07.-12.07. IMC

Normalstation

Eingabe: Intensivstation: 04.07.-12.07.

Gibt es zwischen den Aufenthalten auf der Intensivstation Tage mit Aufenthalt auf der Normalstation, so können wir mehrere verschiedene Intensivstationsaufenthalte eingeben. Die Schlafmittelgaben, die während dem IMC- oder IS-Aufenthalt verabreicht werden, nehmen wir nicht in den Eingabebogen mit auf. Beim Reiter „Tage mit Schlafmitteln" sind die Tage auf der Intensivstation hervorgehoben, um die Intensivstationstage zu sehen und "Nein" anzukreuzen. Mögliche Verschreibungen, die auch vor- oder nach dem Aufenthalt auf der IMC und IS noch relevant sind, nehmen wir aber mit auf. 
Reiter: Diagnosen

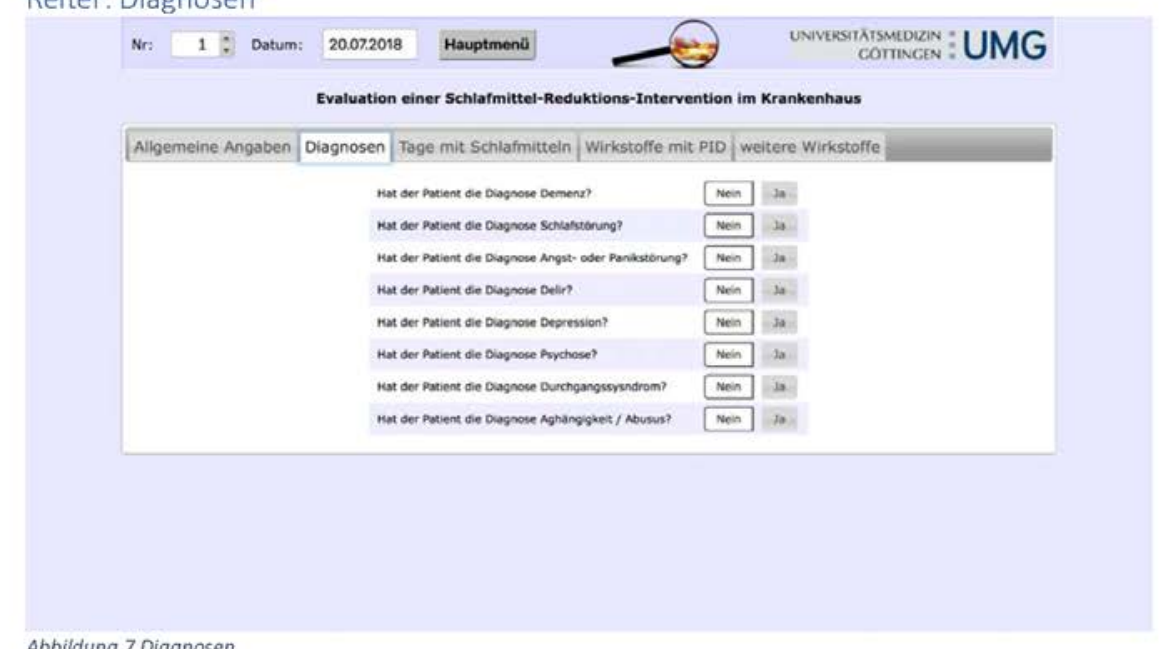

Abbildung 7 Diagnosen

Diagnosen: Hat der Patient eine der Diagnosen, die wir hier auflisten? Wir wählen bei den Diagnosen „Ja" aus, wenn wir davon ausgehen können, dass ein Arzt die Diagnose gestellt hat.

Das sind folgende Informationsquellen:

- Arztbrief des aktuellen Aufenthalts

- Ärztliche Aufnahme-Anamnese des aktuellen Aufenthalts oder die Aufnahme-Anamnese von direkt vorher anschließenden Aufenthalten auf anderen Stationen/ in anderen Krankenhäusern.

- Arztbriefe von vergangenen Krankenhausaufenthalten, die z.B. die Verschreibung eines Antidepressivums erläutern. Beispielsweise könnte das eine "akute depressive Phase“ Phase in einer vergangenen Zeit sein, für die ein Medikament verschrieben wurde, das 
jetzt noch weiter gegeben wird aber die Diagnose möglicherweise nicht bei der Anamnese erfasst wurde oder im aktuellen Arztbrief genannt wird.

- "Verdacht auf Demenz" oder ähnliche Formulierungen über den Verdacht in den ärztlichen Diagnosen zählen wir als Diagnose.

- Diagnosen im Anäthesieprotokoll.

- Diagnosen aus dem Neuropsychologischen Befund.

Diese Informationsquelle zählt nicht:

- Alleinige Erwähnungen von Diagnosen im Anamnesebogen der Gesundheits- und Krankenpfleger können wir nicht als ärztliche Diagnosen zählen.

Wenn der Patient die Diagnose während dem Aufenthalt bekommt und wir das nachverfolgen können, können wir versuchen die Diagnose auch erst ab dem relevanten Zeitpunkt zu vergeben.

Ergänzung 14.11.18: Zu der Diagnose „Durchgangssyndrom“ ist zusätzlich wichtig, ob die Diagnose aufgrund eines aktuellen Geschehens während dem Aufenthalt, den wir dokumentieren, gestellt wurde. Die Diagnose ist für uns wichtig, um beurteilen zu können, ob Medikamentenverordnungen oder -gaben erfolgten oder stattgefunden haben, die diese Diagnose als Begründung haben.

Formulierungen zu Diagnosen, die in den Arztbriefen vorkommen und wie wir sie werten: Da auch die Diagnosen nicht standardisiert in Arztbriefen formuliert sind, müssen wir teilweise im Einzelfall entscheiden, ob die Formulierung eine Diagnose ist oder nicht. Aufgefundene Formulierungen und wie wir sie eingeordnet haben:

\begin{tabular}{|l|l|}
\hline Diagnose & Begriff / Formulierung \\
\hline Angst- oder Panikstörung & $\begin{array}{l}\text { - "Agoraphobie" } \\
\text { - "Panikattacke" }\end{array}$ \\
\hline Demenz & - "Verdacht auf Demenz" \\
\hline Delir & " "teilweise delirant" \\
\hline Depression & - "Dysthymie" \\
\hline Psychose & - "Leichte depressive Episode" \\
\hline
\end{tabular}

Begriffe/Formulierungen, die wir nicht als Diagnosen werten:

- Mild Cognitive Impairment (MCl) - keine Demenz $z^{2}$

- Kognitive Einschränkung ohne Alltagsrelevanz - keine Demenz $z^{2}$

Begriffe/Formulierungen, die wir in die Zuordnungstabelle schreiben müssen:

- Hirnorganisches Psychosyndrom (HOPS) - können wir nicht in unsere Diagnosen einteilen, auch wenn es relevant sein sollte. Die Diagnose HOPS muss in den Kommentaren in der Zuordnungstabelle erwähnt werden.

${ }^{2}$ Deuschl G, Maier W et al. S3-Leitlinie Demenzen. 2016. In: Deutsche Gesellschaft für Neurologie, Hrsg.
Leitlinien für Diagnostik und Therapie in der Neurologie. Online: www.dgn.org/leitlinien (abgerufen am 29.01.2019) 
Reiter: Tage mit Schlafmitteln
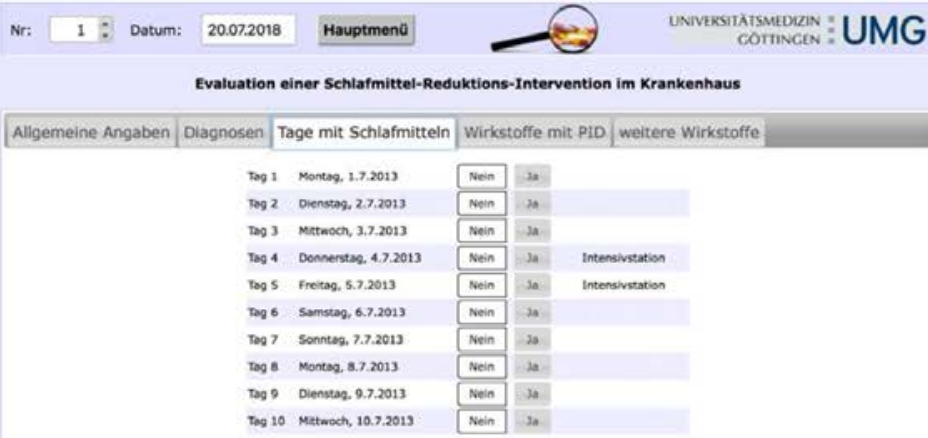

Abbildung 8 Tage mit Schlafmitteln

\section{Tage mit Schlafmitteln:}

Jeden Tag, an dem ein Patient zwischen 18:00-6:00 Uhr ein Schlafmittel erhalten hat (Schlafmittel-Gaben), vermerken wir hier mit „Ja“. Tage, die der Patient auf der IMC/IS verbracht hat zählen hier nicht mit rein und werden mit "Nein" vermerkt.

Generell wichtig zu verabreichten Schlafmitteln:

- Wir beachten alle Schlafmittel, die auf einem Normalstationsaufenthalt zwischen 18:00 abends - 6:00 Uhr morgens verabreicht wurden.

- Wird ein Patient mit einer Dauermedikation nur über das Wochenende nach Hause entlassen, können wir davon ausgehen, dass er die Medikamente mit nach Hause bekommen hat und als verabreicht zählen.

- Steht nur „Ximovan“ bei den Bedarfsmedikamenten und sonst keine Mengenangabe können wir davon ausgehen, dass 1 Tablette gegeben wurde.

- Der Nachtdienst benutzt einen roten Kugelschreiber. Bedarfsmedikation, die mit rot eingetragen wurde, und in den Zeitraum des Nachtdienst passt, können wir als zwischen 18:00 - 6:00 Uhr verabreicht betrachten.

- Es gibt Kurven, die bereits vorgedruckt haben: Nachtmedikation „Noctamid $1 \mathrm{mg}$ bei Bedarf". Bei diesen Kurven können wir davon ausgehen, dass das Medikament verschrieben wurde, da es fest in die Kurven gedruckt ist und so von den Ärzten "abgesegnet" wurde.

- Fluphenazin als Depot zählen wir nicht mit in der Studie (wird nicht als Dauermedikation gezählt) - aber es sollte in den Kommentaren vermerkt werrden, dass der Patient das bekommt. 
Reiter: Wirkstoffe mit PID

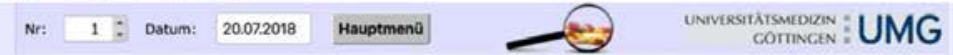

Evaluation einer Schlafmittel-Reduktions-Intervention im Krankenhaus

Allgemeine Angaben Diagnosen Tage mit Schlafmittein Wirkstoffe mit PID | weitere Wirkstoffe

\begin{tabular}{|c|c|c|c|c|c|c|c|c|c|}
\hline Winkstort & Versch & loung & & & & verabreikht en & Savon PID an & (PRD) & Dosity unktiar an \\
\hline Lormetarepom & Nein & onem & Beave & avides. & metranet: & Doen & Dopen & $(>0,5 \mathrm{mg} / \mathrm{c})$ & Dagen \\
\hline Zopicion & Nein & Dent & nater & modes & inteliannt & rogen & Dogen & (> 3,75 mq/d) & ragen \\
\hline Zolpisem & Nein & ower & Beont & nover & enterkamont & trpen & Trapen & (>5 m m/d) & Tragen \\
\hline coraresoum & Nein & Doum & mant & man & indockanont: & ragen & tagen & (>2 mg/d) & Tspen \\
\hline Broticolam & Nein & Dover & ancourt & wisen & anbekaont. & Tragen & Tagen & $(>0,125 \mathrm{mg} / \mathrm{d})$ & rogen \\
\hline nobsentided & Nein & Dower & nosert & never & Unterannt: & Tragen & Tagen & $(>2 \mathrm{~m} / \mathrm{d})$ & ragen \\
\hline Oumasain & Nein & Dover & Boncerd: & mener & mberkans & Trogen & Tagen & $(>10 \mathrm{mo} / \mathrm{\theta})$ & tropen \\
\hline Oxarepam & Nein & Dever & sedent: & wide: & inbeckanes: & ragen & Tragen & $(>60 \mathrm{mo} / \mathrm{\theta})$ & Traen \\
\hline Zolepion & Nein & Oener & and & Beside & intelament & tosen & Tagen & $(25 \mathrm{~m} / \mathrm{d})$ & Troen \\
\hline
\end{tabular}

Abbildung 9 Wirkstoffe mit PID

Verschreibung: Art der Verschreibung: Wurde der Wirkstoff als Dauer-/Bedarf oder beides verschrieben? Wurde der Wirkstoff nicht verschrieben (-> Nein)? Wenn wir nicht klar sehen können, wie ein Medikament verschrieben wurde, können wir "unbekannt“ angeben. Es zählen alle Medikamente, die während dem Aufenthalt auf einer Normalstation verschrieben wurden. Verschreibungen, die während einem Intensivstationsaufenthalt stattgefunden haben zählen auch.

Wenn die PID > $5 \mathrm{mg}$ ist und ein Patient bekommt $5 \mathrm{mg}$ am Tag, dann enstpricht das noch nicht der PID. Erst, wenn die jemand wirklich mehr als $5 \mathrm{mg}$ am Tag, also z.B. 7,5mg bekommt, wird die Gabe an dem Tag als PID gezählt. Zu beachten ist hier, dass weiterhin nur Verordnungen für den Abend (18:00-6:00) und Gaben am Abend (18:00-6:00) gezählt werden.

Wo finden wir Verschreibungen?

Verschreibungen finden wir im Bogen „Verordnungen“. Dort können wir sowohl Dauer- als auch Bedarfsmedikation finden.

Verabreichungen / Gaben:

Verabreicht an: An wie vielen Tagen wurde der Wirkstoff verabreicht?

Es zählen alle Verabreichungen, die auf einem Normalstationsaufenthalt zwischen 18:00 und 6:00 stattgefunden haben.

Wurden die Medikamente in potenziell inadäquater Dosierung (PID) verabreicht?

An wie vielen Tagen wurde der Wirkstoff insgesamt verabreicht? An wie vielen Tagen davon als PID? An wie vielen Tagen war die Dosierung nicht klar herauszulesen?

Wo finden wir die Verabreichungen/Gaben?

In den Patientenkurven sind die Dauer- und Bedarfsmedikation vermerkt.

Dauermedikation wird oft mit dem Vermerk „siehe Planette“ oder ähnlich beschrieben.

Dann gilt als Dauermedikation der Bogen mit den ärztlichen Verordnungen. Manchmal wird 
die Dauermedikation auch klar in die Kurve geschrieben. Dann können wir uns an der Kurve und den Verordnungen orientieren.

Bedarfsmedikation wird für jeden Tag einzeln eingetragen. In der Kurve finden wir je nach Ausführung (es gibt unterschiedliche) die Zeile „Bedarfsmedikation" oder auch die Zeilen „Nachtmedikation"/ „Schlafmedikamente" oder ähnliche.

Hier wichtig: Es kann sein, dass ein Medikament verschrieben wurde aber dann nicht verabreicht. Genauso kann es sein, dass ein Medikament nicht verschrieben wurde, aber trotzdem verabreicht. Das wollen wir festhalten mit dieser Maske.

Es kann auch sein, dass die Medikamente in der Kurve nicht klar weitergeführt werden bis zum Ende eines Aufenthalts. Hier können wir, wenn es nicht anders vermerkt ist, davon ausgehen, dass die Medikamente dann bis Ende des Aufenthalts weitergegeben wurden.

Reiter: Weitere Wirkstoffe

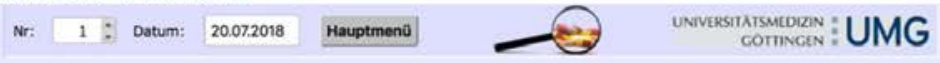

Evaluation einer Schlafmittel-Reduktions-Intervention im Krankenhaus

Allgemeine Angaben Dlagnosen Tage mit Schlafmittein | Wirkstoffe mit PID weitere Wirkstoffe

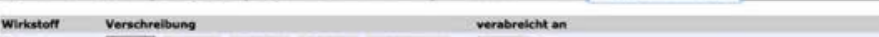

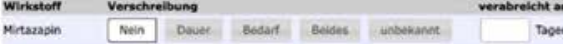

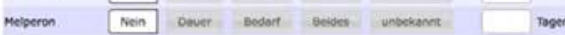

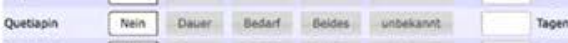

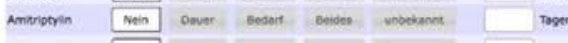

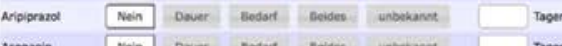

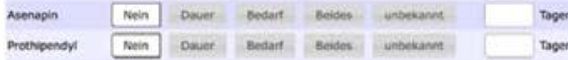

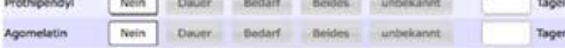

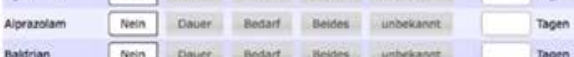

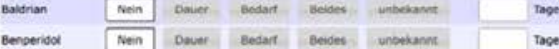

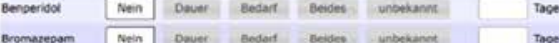

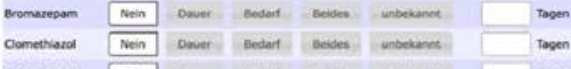

Abbildung 10 Weitere Wirkstoffe

Weitere Wirkstoffe:

Verschreibung: Art der Verschreibung: Wurde der Wirkstoff als Dauer-/Bedarf oder beides verschrieben?

Verabreicht an: An wie vielen Tagen wurde der Wirkstoff verabreicht?

Gleiches Vorgehen wie bei "Wirkstoffe mit PID“, außer dass wir nicht auf die Dosierung achten. 
Wirkstoffliste

Wir haben eine Wirkstoffliste mit allen bestellten Wirkstoffen im Zeitraum 2013 und 2017. Der Vorteil dieser Liste ist, dass wir Einträge in den Akten wie „Stilnox" nachgucken können und damit wissen, was mit der Bezeichnung gemeint ist.

Beispiel:

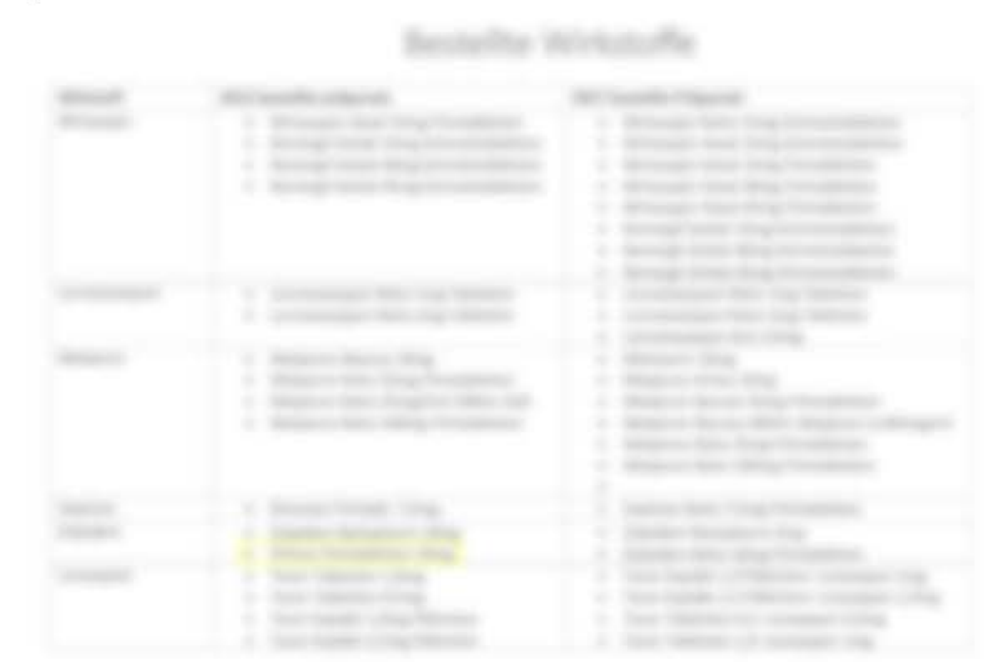

Abbildung 11 Ausschnitt aus der Wirkstoffliste (24.04.19: für die Veröffentlichung weichgezeichnet)

Wenn in der Akte "Stilnox" verschrieben wird, können wir davon ausgehen, dass damit Zolpidem in der Dosierung 10mg gemeint ist.

Am Ende der Wirkstoffliste ist eine Liste mit häufig benutzten Begriffen / Abkürzungen, die benutzt werden, um einen Wirkstoff zu benennen.

Als Beispiel: „Noctamid“ ist der Name für ein Lormetazepam-Präparat, das aber vom Krankenhaus Weende weder 2013 noch 2017 bestellt wurde. Wir können davon ausgehen, dass der Name Noctamid einfach sehr gebräuchlich ist und deswegen vom Personal benutzt wird, als synonym für Lormetazepam. 
JONAS KLEMPERER

8.3.19

STANDARD OPERATING PROCEDURE - WEENDE

Abkürzungen

\begin{tabular}{|l|l|}
\hline Abkürzung & Bedeutung \\
\hline EKW & Evangelisches Krankenhaus Weende \\
\hline Abteilungen & Allgemeinchirurgie \\
\hline ACH & Unfallchirurgie \\
\hline UCH & Urologie \\
\hline UR & Innere Medizin \\
\hline IM & Innere Medizin Weende \\
\hline IMW & Schmerztherapie \\
\hline SHT & Geriatrische Tagesklinik \\
\hline GTK & Plastische Chirurgie \\
\hline PCH & Geriatrische Akutstation \\
\hline GA & Geriatrische Rehabilitation \\
\hline GR & Intensivstation \\
\hline IS / ITS / IST & Intermediate Care \\
\hline IMC & \\
\hline
\end{tabular}


JONAS KLEMPERER

STANDARD OPERATING PROCEDURE - WEENDE

Fragestellungen, die aufkamen und wie wir sie gelöst haben:

Wo? Problem?

Lösung?

Informationen für die Veröffentlichung redigiert.

Log der Ergänzungen

Informationen für die Veröffentlichung redigiert. 


\subsection{Verteilung von Diagnosen nach Abteilung}

In Tabelle A 6 ist die Verteilung von Patienten mit neurologisch-psychiatrischen Diagnosen in den drei Fachbereichen in Prä- und Postinterventionszeitraum dargestellt.

Patienten der Geriatrie machen bei fast allen Diagnosen den größten Anteil in beiden Zeiträumen aus. Patienten der Inneren Medizin haben am zweithäufigsten neurologisch-psychiatrische Diagnosen. Eine Ausnahme gibt es hier bei der Diagnose Delir, die bei Patienten der Chirurgie häufiger ist als bei Patienten der Inneren Medizin. Hatte ein Patient mehrere Diagnosen kommt er in der Tabelle mehrmals vor.

Tabelle A 6 Patienten mit neurologisch-psychiatrischen Diagnosen nach Abteilung

\begin{tabular}{|c|c|c|c|c|c|c|}
\hline \multirow[b]{3}{*}{ In $\%$} & \multicolumn{3}{|c|}{ Prä-Intervention } & \multicolumn{3}{|c|}{ Post-Intervention } \\
\hline & Innere & Chirurgie & Geriatrie & Innere & Chirurgie & Geriatrie \\
\hline & $\mathrm{n}=383$ & $\mathrm{n}=395$ & $\mathrm{n}=182$ & $\mathrm{n}=389$ & $\mathrm{n}=428$ & $\mathrm{n}=232$ \\
\hline Demenz & 16,2 & 7,6 & 22,5 & 9,8 & 9,1 & 22,4 \\
\hline Depression & 8,4 & 6,3 & 19,8 & 8,5 & 3,7 & 15,9 \\
\hline Delir & 0,5 & 1,0 & 6,0 & 1,0 & 2,1 & 2,6 \\
\hline $\begin{array}{l}\text { Abhängig- } \\
\text { keit }\end{array}$ & 1,0 & 0,5 & 3,8 & 1,3 & 0,0 & 1,7 \\
\hline $\begin{array}{l}\text { Angst oder } \\
\text { Panik }\end{array}$ & 1,6 & 1,0 & 1,6 & 1,8 & 1,2 & 4,3 \\
\hline Psychose & 0,5 & 0,5 & 0,5 & 1,0 & 1,4 & 2,2 \\
\hline $\begin{array}{l}\text { Schlafstö- } \\
\text { rung }\end{array}$ & 0,5 & 0,5 & 1,1 & 0,5 & 0,7 & 0,4 \\
\hline Andere* & 0 & 0 & 0 & 1,3 & 0,5 & 1,7 \\
\hline
\end{tabular}

*Andere neurologisch-psychiatrische Diagnosen, z. B. affektive Störung 


\subsection{Ergebnisse mit Signifikanzwerten, Odds Ratios und Konfidenzintervallen}

Tabelle A 7 Anteil der Patienten mit schlafanstoßenden Medikamenten und Anteil der Patienten, die Benzodiazepine, Z-Drugs, Antidepressiva, Antipsychotika oder Baldrian erhielten. Jeweils im Vergleich der beiden Zeiträume 2013 und 2017 im Interventions- und Kontrollkrankenhaus

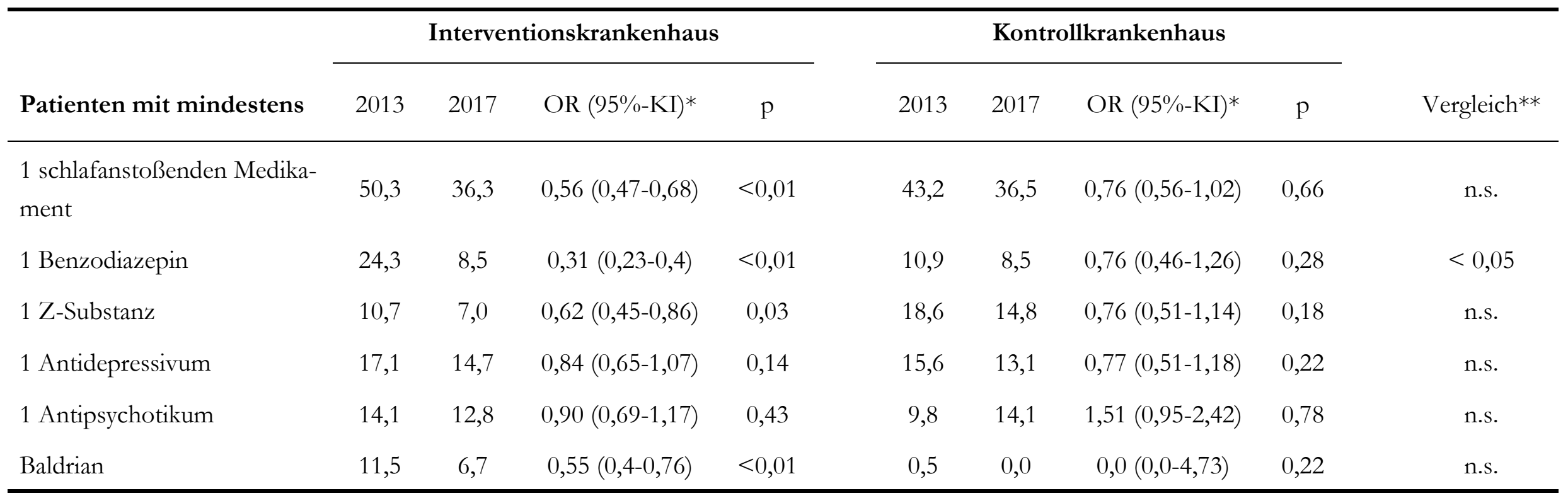

* 95\%-Konfidenzintervall

** Vergleich zwischen Interventions- und Kontrollkrankenhaus (Wenn KI nicht überlappen: $\mathrm{p}<0,05$ ) 


\subsection{Vollständige Tabelle aller erhobenen Wirkstoffe}

Tabelle A 8 ungekürzte Übersicht aller Patienten nach Kontakt mit schlafanstoßenden Medikamenten. PID: Potenziell inadäquate Dosierung; J.G.: Jede Gabe; n.a.: nicht anwendbar.

\begin{tabular}{|c|c|c|c|c|c|c|}
\hline & \multicolumn{3}{|c|}{$\begin{array}{l}\text { Prä-Intervention } \\
\qquad(\mathrm{n}=960 \text { Pat.) }\end{array}$} & \multicolumn{3}{|c|}{$\begin{array}{l}\text { Post-Intervention } \\
\qquad(\mathrm{n}=1049 \text { Pat.) }\end{array}$} \\
\hline & $\mathbf{n}$ & $\%$ & PID (\%) & $\mathbf{n}$ & $\%$ & PID (\%) \\
\hline Keine schlafanst. Medik. & 480 & 50,0 & & 667 & 63,6 & \\
\hline Alle schlafanst. Medik. & 483 & 50,3 & & 381 & 36,3 & \\
\hline Ohne Baldrian & 440 & 45,8 & & 343 & 32,7 & \\
\hline Benzodiazepine & 233 & 24,3 & & 89 & 8,5 & \\
\hline Lormetazepam & 127 & 13,2 & $>0,5 \mathrm{mg}(11,7)$ & 23 & 2,2 & $>0,5 \mathrm{mg}(1,5)$ \\
\hline Lorazepam & 55 & 5,7 & $>2 \mathrm{mg}(0,2)$ & 46 & 4,4 & $>2 \mathrm{mg}(0,4)$ \\
\hline Oxazepam & 18 & 1,9 & $>60 \mathrm{mg}(0,1)$ & 6 & 0,6 & $>60 \mathrm{mg}(0)$ \\
\hline Diazepam & 14 & 1,5 & J.G. $(1,5)$ & 2 & 0,2 & J.G. $(0,2)$ \\
\hline Temazepam & 7 & 0,7 & J.G. $(0,7)$ & 3 & 0,3 & J.G. $(0,3)$ \\
\hline Tetrazepam & 3 & 0,3 & J.G. $(0,3)$ & 0 & 0,0 & J.G. (0) \\
\hline Dikaliumclorazepat & 3 & 0,3 & J.G. $(0,3)$ & 1 & 0,1 & J.G. $(0,1)$ \\
\hline Flunitrazepam & 3 & 0,3 & J.G. $(0,3)$ & 0 & 0,0 & J.G. (0) \\
\hline Bromazepam & 2 & 0,2 & J.G. $(0,2)$ & 5 & 0,5 & J.G. $(0,5)$ \\
\hline Flurazepam & 1 & 0,1 & J.G. $(0,1)$ & 1 & 0,1 & J.G. $(0,1)$ \\
\hline Nitrazepam & 0 & 0,0 & n.a. & 2 & 0,2 & n.a. \\
\hline Z-Substanzen & 103 & 10,7 & & 73 & 7,0 & \\
\hline Zopiclon & 66 & 6,9 & $>7,5 \mathrm{mg}(3,8)$ & 25 & 2,4 & $>7,5 \mathrm{mg}(1,7)$ \\
\hline Zolpidem & 37 & 3,9 & $>5 \mathrm{mg}(2,7)$ & 48 & 4,6 & $>5 \mathrm{mg}(2,6)$ \\
\hline Neuroleptika & 135 & 14,1 & & 134 & 12,8 & \\
\hline Melperon & 53 & 5,5 & n.a. & 50 & 4,8 & n.a. \\
\hline Quetiapin & 27 & 2,8 & n.a. & 48 & 4,6 & n.a. \\
\hline Prothipendyl & 19 & 2,0 & n.a. & 11 & 1,0 & n.a. \\
\hline Promethazin & 14 & 1,5 & n.a. & 1 & 0,1 & n.a. \\
\hline Haloperidol & 8 & 0,8 & $>2 \mathrm{mg}(0,2)$ & 3 & 0,3 & $>2 \mathrm{mg}(0,1)$ \\
\hline Risperidon & 7 & 0,7 & n.a. & 12 & 1,1 & n.a. \\
\hline Olanzapin & 4 & 0,4 & $>10 \mathrm{mg}(0,2)$ & 4 & 0,4 & $>10 \mathrm{mg}(0,2)$ \\
\hline Benperidol & 2 & 0,2 & n.a. & 0 & 0,0 & n.a. \\
\hline Pipamperon & 1 & 0,1 & n.a. & 5 & 0,5 & n.a. \\
\hline
\end{tabular}




\begin{tabular}{|c|c|c|c|c|c|c|}
\hline & \multicolumn{3}{|c|}{$\begin{array}{l}\text { Prä-Intervention } \\
\qquad(\mathrm{n}=960 \text { Pat.) }\end{array}$} & \multicolumn{3}{|c|}{$\begin{array}{l}\text { Post-Intervention } \\
\text { (n = 1049 Pat.) }\end{array}$} \\
\hline & $\mathrm{n}$ & $\%$ & PID (\%) & $\mathrm{n}$ & $\%$ & PID (\%) \\
\hline Antidepressiva & 164 & 17,1 & & 154 & 14,7 & \\
\hline Mirtazapin & 130 & 13,5 & n.a. & 137 & 13,1 & n.a. \\
\hline Amitriptylin & 11 & 1,1 & J.G. $(1,1)$ & 9 & 0,9 & J.G. $(0,9)$ \\
\hline Doxepin & 8 & 0,8 & J.G. $(0,8)$ & 4 & 0,4 & J.G. $(0,4)$ \\
\hline Opipramol & 4 & 0,4 & n.a. & 1 & 0,1 & n.a. \\
\hline Trimipramin & 4 & 0,4 & J.G. $(0,4)$ & 1 & 0,1 & J.G. $(0,1)$ \\
\hline Mianserin & 2 & 0,2 & n.a. & 1 & 0,1 & n.a. \\
\hline Agomelatin & 1 & 0,1 & n.a. & 0 & 0,0 & n.a. \\
\hline Maprotilin & 1 & 0,1 & J.G. $(0,1)$ & 0 & 0,0 & J.G. (0) \\
\hline Trazodon & 0 & 0,0 & n.a. & 1 & 0,1 & n.a. \\
\hline Andere & 111 & 11,6 & & 71 & 6,8 & \\
\hline Baldrian & 110 & 11,5 & n.a. & 70 & 6,7 & n.a. \\
\hline Melatonin & 1 & 0,1 & n.a. & 1 & 0,1 & n.a. \\
\hline
\end{tabular}




\subsubsection{Wirkstoffe ohne Verabreichungen}

Aus der breiten Auswahl an Wirkstoffen, die in der Studie betrachtet wurden, konnten bei einigen Wirkstoffen keine Verabreichungen festgestellt werden (Tabelle A 9).

Tabelle A 9 Wirkstoffe, von denen keine Verabreichung festgestellt werden konnte

\begin{tabular}{|c|c|c|c|c|}
\hline $\begin{array}{c}\text { Benzodiaze- } \\
\text { pine }\end{array}$ & Z-Substanzen & Neuroleptika & $\begin{array}{c}\text { Antidepres- } \\
\text { siva }\end{array}$ & Andere \\
\hline Alprazolam & Zaleplon & Aripiprazol & $\begin{array}{l}\text { Clomethia- } \\
\text { zol }\end{array}$ & $\begin{array}{l}\text { Diphenhyd- } \\
\text { ramin }\end{array}$ \\
\hline Brotizolam & & Asenapin & & Doxylamin \\
\hline Clobazam & & Chloralhydrat & & \\
\hline Medazepam & & Chlordiazepoxid & & \\
\hline Prazepam & & Chlorprothixen & & \\
\hline \multirow[t]{8}{*}{ Triazolam } & & Clozapin & & \\
\hline & & Flupentixol & & \\
\hline & & Fluphenazin & & \\
\hline & & Levomepromazin & & \\
\hline & & Perazin & & \\
\hline & & Perphenazin & & \\
\hline & & Thioridazin & & \\
\hline & & Zuclopenthixol & & \\
\hline
\end{tabular}




\section{$9 \quad$ Literaturverzeichnis}

Abi-Ackel MM, Lima-Costa MF, Castro-Costa É, Loyola Filho AI de (2017): Psychotropic drug use among older adults: prevalence and associated factors. Rev Bras Epidemiol 20, 57-69

Allain H, Bentué-Ferrer D, Polard E, Akwa Y, Patat A (2005): Postural instability and consequent falls and hip fractures associated with use of hypnotics in the elderly. Drugs Aging 22, 749-765

Alldred DP, Kennedy MC, Hughes C, Chen TF, Miller P (2016): Interventions to optimise prescribing for older people in care homes. Cochrane Database Syst Rev 2, CD009095

American Geriatrics Society (2015): Ten Things Clinicians and Patients Should Question. http://www.choosingwisely.org/societies/american-geriatrics-society; Zugriff am 13.09.2019.

Anthierens S, Habraken H, Petrovic M, Christiaens T (2007): The lesser evil? Initiating a benzodiazepine prescription in general practice. Scand J Prim Health Care 25, 214-219

Arnold I: Verordnung von Antidepressiva und Neuroleptika bei $\geq 65$-Jährigen in einem Krankenhaus der Grund- und Regelversorgung. Med. Diss. Göttingen 2017

Arnold I, Straube K, Himmel W, Heinemann S, Weiss V, Heyden L, Hummers-Pradier E, Nau R (2017): High prevalence of prescription of psychotropic drugs for older patients in a general hospital. BMC Pharmacol Toxicol 18, 76

Avorn J, Soumerai SB, Everitt DE, Ross-Degnan D, Beers MH, Sherman D, Salem-Schatz SR, Fields D (1992): A randomized trial of a program to reduce the use of psychoactive drugs in nursing homes. N Engl J Med $\underline{327}, 168-173$

Badr AF, Kurdi S, Alshehri S, McManus C, Lee J (2018): Pharmacists' interventions to reduce sedative/hypnotic use for insomnia in hospitalized patients. Saudi Pharm J 26, 1204-1207

Bartels R, Kohlstedde M (2017): Qualitätsbericht 2017 - St. Martini gGmbH Krankenhaus Duderstadt. https://g-ba-qualitaetsberichte.de/api/file/download/260310334-00-2017-xml.pdf; Zugriff am 17.08.2019.

Beers MH (1997): Explicit criteria for determining potentially inappropriate medication use by the elderly. An update. Arch Intern Med 157, 1531-1536

Beers MH, Munekata M, Storrie M (1990): The accuracy of medication histories in the hospital medical records of elderly persons. J Am Geriatr Soc $\underline{38}, 1183-1187$

Beers MH, Ouslander JG, Rollingher I, Reuben DB, Brooks J, Beck JC (1991): Explicit criteria for determining inappropriate medication use in nursing home residents. Arch Intern Med 151, 1825-1832

Benkert O, Hippius H (Hrsg.): Kompendium der Psychiatrischen Pharmakotherapie. 12. Auflage; Springer, Berlin 2019

Bryant PA, Trinder J, Curtis N (2004): Sick and tired: does sleep have a vital role in the immune system? Nat Rev Immunol 4 , 457-467

Caglar S, Henneman PL, Blank FS, Smithline HA, Henneman EA (2011): Emergency department medication lists are not accurate. J Emerg Med 40, 613-616 
Canadian Geriatrics Society (2017): Choosing Wisely Canada - Five things physicians and patients should question. https://choosingwiselycanada.org/geriatrics; Zugriff am 13.09.2019.

Chung S, Youn S, Park B, Lee S, Kim C (2018): A sleep education and hypnotics reduction program for hospitalized patients at a general hospital. Psychiatry Investig $\underline{15}, 78-83$

Cohrs S (2008): Sleep disturbances in patients with schizophrenia. CNS Drugs 22, 939-962

Corbi G, Gambassi G, Pagano G, Russomanno G, Conti V, Rengo G, Leosco D, Bernabei R, Filippelli A, Ferrara N (2015): Impact of an innovative educational strategy on medication appropriate use and length of stay in elderly patients. Medicine (Baltimore) 94, e918

Coupland CAC, Hill T, Dening T, Morriss R, Moore M, Hippisley-Cox J (2019): Anticholinergic drug exposure and the risk of dementia: a nested case-control study. JAMA Intern Med $\underline{179}$, 1084-1093

Dalton K, O’Brien G, O'Mahony D, Byrne S (2018): Computerised interventions designed to reduce potentially inappropriate prescribing in hospitalised older adults: a systematic review and meta-analysis. Age Ageing 47, 670-678

Dirnberger G (2020): Pschyrembel Online - Polysomnographie. https://www.pschyrembel.de/polysomnographie/A06CJ/doc; Zugriff am 27.12.2020.

Dobing S, Frolova N, McAlister F, Ringrose J (2016): Sleep quality and factors influencing self-reported sleep duration and quality in the general internal medicine inpatient population. PLoS ONE $11, \mathrm{e} 0156735$

Dolan C, Omer S, Glynn D, Corcoran M, McCarthy G (2012): Benzodiazepine and z-drug prescribing for elderly people in a general hospital: a complete audit cycle. Ir J Psychol Med $\underline{29}$, $128-131$

Döring N, Bortz J: Untersuchungsdesign. In: Döring N, Bortz J (Hrsg.): Forschungsmethoden und Evaluation in den Sozial- und Humanwissenschaften (Springer-Lehrbuch). Springer, Berlin 2016, 181-220

Eide E, Schjøtt J (2001): Assessing the effects of an intervention by a pharmacist on prescribing and administration of hypnotics in nursing homes. Pharm World Sci $\underline{23}, 227-231$

Elliott RA, Woodward MC, Oborne CA (2001): Improving benzodiazepine prescribing for elderly hospital inpatients using audit and multidisciplinary feedback. Intern Med J $\underline{31}, 529-535$

Endres HG, Kaufmann-Kolle P, Knopf H, Thürmann PA (2018): Welche Faktoren begünstigen die Anwendung potenziell ungeeigneter Medikamente bei älteren Menschen? Bundesgesundheitsblatt - Gesundheitsforschung - Gesundheitsschutz $\underline{61}, 40-51$

Enzensperger C, Lehmann J (2007): Neuroleptika und Antidepressiva als Hypnotika. Wenn Nebenwirkungen zur Hauptwirkung werden. Pharm Unserer Zeit $\underline{36}$, 196-200

Everitt H, McDermott L, Leydon G, Yules H, Baldwin D, Little P (2014): GPs' management strategies for patients with insomnia: a survey and qualitative interview study. Br J Gen Pract $\underline{64}$, e112-e119

Everitt H, Baldwin DS, Stuart B, Lipinska G, Mayers A, Malizia AL, Manson CC, Wilson S (2018): Antidepressants for insomnia in adults. Cochrane Database Syst Rev $\underline{5}$, CD010753 
Faul F, Erdfelder E, Lang AG, Buchner A (2007): G*Power 3: A flexible statistical power analysis program for the social, behavioral, and biomedical sciences. Behav Res Methods $\underline{39}$, 175-191

Feinsilver SH, Hernandez AB (2017): Sleep in the elderly: unanswered questions. Clin Geriatr Med $\underline{33}, 579-596$

Fick DM, Cooper JW, Wade WE, Waller JL, Maclean JR, Beers MH (2003): Updating the Beers Criteria for potentially inappropriate medication use in older adults: results of a US consensus panel of experts. Arch Intern Med 163, 2716-2724

Fillary J, Chaplin H, Jones G, Thompson A, Holme A, Wilson P (2015): Noise at night in hospital general wards: a mapping of the literature. Br J Nurs $24,536-540$

Finkle WD, Der JS, Greenland S, Adams JL, Ridgeway G, Blaschke T, Wang Z, Dell RM, VanRiper KB (2011): Risk of fractures requiring hospitalization after an initial prescription for zolpidem, alprazolam, lorazepam, or diazepam in older adults. J Am Geriatr Soc 모, 1883-1890

Gage SB de, Bégaud B, Bazin F, Verdoux H, Dartigues JF, Pérès K, Kurth T, Pariente A (2012): Benzodiazepine use and risk of dementia: prospective population based study. BMJ $\underline{345}$, e6231

Giron MST, Forsell Y, Bernsten C, Thorslund M, Winblad B, Fastbom J (2001): Psychotropic drug use in elderly people with and without dementia. Int J Geriatr Psychiatry 16, 900-906

Glass J, Lanctôt KL, Herrmann N, Sproule BA, Busto UE (2005): Sedative hypnotics in older people with insomnia: meta-analysis of risks and benefits. BMJ $\underline{331}, 1169$

Gogol M (2016): Klug entscheiden: . . in der Geriatrie. Dtsch Arztebl Int 113, A-1756-A-1760

Gray SL, Dublin S, Yu O, Walker R, Anderson M, Hubbard RA, Crane PK, Larson EB (2016):

Benzodiazepine use and risk of incident dementia or cognitive decline: prospective population based study. BMJ $\underline{352}$, i90

Gurwitz JH, Rochon P (2002): Improving the quality of medication use in elderly patients: a not-sosimple prescription. Arch Intern Med 162, 1670-1672

Hackenfort L, Kohlstedde M (2013): Qualitätsbericht 2013 - St. Martini gGmbH Krankenhaus Duderstadt. https://g-ba-qualitaetsberichte.de/api/file/download/260310334-00-2013-xml.pdf; Zugriff am 17.08.2019.

Hampel T, Karaus M (2017): Qualitätsbericht 2017 - Evangelisches Krankenhaus Göttingen-Weende gGmbH. https://g-ba-qualitaetsberichte.de/api/file/download/260310447-00-2017xml.pdf; Zugriff am 26.04.2019.

Heinemann S, Weiß V (2016): Universitätsmedizin Göttingen - Schlaffreundliches Krankenhaus. http://www.schlaffreundliches-krankenhaus.de; Zugriff am 27.12.2020.

Heinemann S, Weiß V, Straube K, Nau R, Grimmsmann T, Himmel W, Hummers-Pradier E (2016): Understanding and reducing the prescription of hypnotics and sedatives at the interface of hospital care and general practice: a protocol for a mixed-methods study. BMJ Open $\underline{6}$, e011908

Heinemann S, Neukirchen F, Nau R, Hummers E, Himmel W (2019): Patient-reported factors associated with the desire to continue taking sleep-inducing drugs after hospital discharge: A survey of older adults. Pharmacoepidemiol Drug Saf $\underline{28}, 1014-1022$ 
Hertel S, Klug J, Schmitz B: Quasi-experimentelle Versuchspläne. In: Handbuch Statistik, Methoden und Evaluation (Handbuch der Psychologie). Band 13; Hogrefe, Göttingen 2010, 49-62

Hoffmann F, Glaeske G (2014): Benzodiazepinhypnotika, Zolpidem und Zopiclon auf Privatrezept. Nervenarzt $\underline{85}, 1402-1409$

Holt S, Schmiedl S, Thürmann PA (2010): Potenziell inadäquate Medikation für ältere Menschen: die PRISCUS-Liste. Dtsch Ärztebl Int 107, 543-551

Holt S, Schmiedl S, Thürmann PA (2011): PRISCUS-Liste potenziell inadäquater Medikation für ältere Menschen. https://media.gelbe-liste.de/documents/priscus-liste.pdf; Zugriff am 26.12.2020.

Hu RF, Jiang XY, Chen J, Zeng Z, Chen XY, Li Y, Huining X, Evans DJ, Wang S (2015): Nonpharmacological interventions for sleep promotion in the intensive care unit. Cochrane Database Syst Rev 10, CD008808

Kanji S, Mera A, Hutton B, Burry L, Rosenberg E, MacDonald E, Luks V (2016): Pharmacological interventions to improve sleep in hospitalised adults: a systematic review. BMJ Open $\underline{6}$, e012108

Kern WV (2018): Antibiotic stewardship-Programme in Akutkrankenhäusern. Klin 4ㄱ, 272-276

Kim JS, Choi S, Kwon SU, Seo YS (2002): Inability to control anger or aggression after stroke. Neurology $\underline{58}, 1106-1108$

Knauert MP, Pisani MA: Chapter 19 - Sleep in hospitalized patients. In: Savard J, Ouellet MC (Hrsg.): Handbook of sleep disorders in medical conditions. Academic Press, London 2019, $411-437$

Kripke DF, Langer RD, Kline LE (2012): Hypnotics' association with mortality or cancer: a matched cohort study. BMJ Open 2, e000850

Kruse WHH (1990): Problems and pitfalls in the use of benzodiazepines in the elderly. Drug Saf $\underline{5}$, 328-344

Lader M (2011): Benzodiazepines revisited - will we ever learn? Addiction 106, 2086-2109

Leape LL (2014): The checklist conundrum. N Engl J Med $\underline{370,1063-1064}$

Lohse MJ, Müller-Oerlinghausen B: Hypnotika und Sedativa. In: Schwabe U, Paffrath D (Hrsg.): Arzneiverordnungs-Report 2014. Springer, Berlin 2014a, 701-715

Lohse MJ, Müller-Oerlinghausen B: Psychopharmaka. In: Schwabe U, Paffrath D (Hrsg.): Arzneiverordnungs-Report 2014. Springer, Berlin 2014b, 921-965

Lohse MJ, Müller-Oerlinghausen B: Hypnotika und Sedativa. In: Schwabe U, Paffrath D, Ludwig W-D, Klauber J (Hrsg.): Arzneiverordnungs-Report 2018. Springer, Berlin 2018a, 553-562

Lohse MJ, Müller-Oerlinghausen B: Psychopharmaka. In: Schwabe U, Paffrath D, Ludwig W-D, Klauber J (Hrsg.): Arzneiverordnungs-Report 2018. Springer, Berlin 2018b, 733-761

Morlock RJ, Tan M, Mitchell DY (2006): Patient characteristics and patterns of drug use for sleep complaints in the United States: analysis of national ambulatory medical survey data, 19972002. Clin Ther $28,1044-1053$ 
Mostofian F, Ruban C, Simunovic N, Bhandari M (2015): Changing physician behavior: what works? Am J Manag Care 21, 75-84

Mugunthan K, McGuire T, Glasziou P (2011): Minimal interventions to decrease long-term use of benzodiazepines in primary care: a systematic review and meta-analysis. Br J Gen Pract $\underline{61}$, e573-e578

Müller MJ, Benkert O: Antipsychotika. In: Benkert O, Hippius H (Hrsg.): Kompendium der Psychiatrischen Pharmakotherapie. Springer, Berlin 2019, 284-504

Neukirchen F: Schlaf um jeden Preis Eine Befragung älterer Patienten zu den Erfahrungen mit Schlaf- und Beruhigungsmitteln im Krankenhaus und zu Hause. Med. Diss. Göttingen 2018

O’Connor MN, Gallagher P, O’Mahony D (2012): Inappropriate prescribing: criteria, detection and prevention. Drugs Aging 29, 437-452

O’Mahony D, O’Sullivan D, Byrne S, O’Connor MN, Ryan C, Gallagher P (2015): STOPP/START criteria for potentially inappropriate prescribing in older people: version 2. Age Ageing 44, 213-218

Opedal K, Schjøtt J, Eide E (1998): Use of hypnotics among patients in geriatric institutions. Int J Geriatr Psychiatry 13, 846-851

O’Reilly R, Rusnak C (1990): The use of sedative-hypnotic drugs in a university teaching hospital. CMAJ Can Med Assoc J 142, 585-589

Panacek EA (2007): Performing chart review studies. Air Med J 26, 206-210

Pariente A, Gage SB de, Moore N, Bégaud B (2016): The benzodiazepine-dementia disorders link: current state of knowledge. CNS Drugs $\underline{30}, 1-7$

Pimlott NJG, Hux JE, Wilson LM, Kahan M, Li C, Rosser WW (2003): Educating physicians to reduce benzodiazepine use by elderly patients: a randomized controlled trial. CMAJ $\underline{168}, 835-$ 839

Pitkala KH, Juola AL, Hosia H, Teramura-Gronblad M, Soini H, Savikko N, Bell JS (2015): Eightyear trends in the use of opioids, other analgesics, and psychotropic medications among institutionalized older people in Finland. J Am Med Dir Assoc 16, 973-978

Pohl-Dernick K, Meier F, Maas R, Schöffski O, Emmert M (2016): Potentially inappropriate medication in the elderly in Germany: an economic appraisal of the PRISCUS list. BMC Health Serv Res 16,109

Rankin A, Cadogan CA, Patterson SM, Kerse N, Cardwell CR, Bradley MC, Ryan C, Hughes C (2018): Interventions to improve the appropriate use of polypharmacy for older people. Cochrane Database Syst Rev 2, CD008165

Reeve E, Ong M, Wu A, Jansen J, Petrovic M, Gnjidic D (2017): A systematic review of interventions to deprescribe benzodiazepines and other hypnotics among older people. Eur J Clin Pharmacol 73, 927-935

Regen F, Benkert O: Antidepressiva. In: Benkert O, Hippius H (Hrsg.): Kompendium der Psychiatrischen Pharmakotherapie. Springer, Berlin 2019, 1-208 
Renom-Guiteras A, Meyer G, Thürmann PA (2015): The EU(7)-PIM list: a list of potentially inappropriate medications for older people consented by experts from seven European countries. Eur J Clin Pharmacol 71, 861-875

Riemann D, Nissen C: Sleep and Psychotropic Drugs. In: Morin CM, Espie CA (Hrsg.): The Oxford Handbook of Sleep and Sleep Disorders (Oxford Handbooks). Oxford University Press, New York 2012

Riemann D, Baum E, Cohrs S, Crönlein T, Hajak G, Hertenstein E, Klose P, Langhorst J, Mayer G, Nissen C, et al. (2017): S3-Leitlinie Nicht erholsamer Schlaf/Schlafstörungen. Somnologie 21, 2-44

Saß AC, Wurm S, Ziese T: Somatische und psychische Gesundheit. In: Böhm K, Tesch Römer C, Ziese T (Hrsg.): Gesundheit und Krankheit im Alter (Beiträge zur Gesundheitsberichterstattung des Bundes). Gesundheitsberichterstattung des Bundes, Berlin 2009, 323

Schwabe U, Paffrath D, Ludwig WD, Klauber J (Hrsg.): Arzneiverordnungs-Report 2017: aktuelle Daten, Kosten, Trends und Kommentare. Springer, Berlin 2017

Siebert S, Elkeles B, Hempel G, Kruse J, Smollich M (2013): Die PRISCUS-Liste im klinischen Test. Z Für Gerontol Geriatr $\underline{46}$, 35-47

Smith AJ, Tett SE (2010): Improving the use of benzodiazepines - is it possible? A non-systematic review of interventions tried in the last 20 years. BMC Health Serv Res $\underline{10}, 321$

Somers A, Robays H, Audenaert K, Van Maele G, Bogaert M, Petrovic M (2011): The use of hypnosedative drugs in a university hospital: has anything changed in 10 years? Eur J Clin Pharma$\operatorname{col} \underline{67}, 723-729$

Statistisches Bundesamt (2013): Diagnosedaten der Krankenhauspatientinnen und -patienten 2013. https://www.destatis.de/GPStatistik/servlets/MCRFileNodeServlet/DEHeft_derivate_00015405/5231301137015.xlsx; Zugriff am 14.07.2019.

Statistisches Bundesamt (2017): Diagnosedaten der Krankenhauspatientinnen und -patienten 2017. https://www.destatis.de/DE/Themen/Gesellschaft-Umwelt/Gesundheit/Krankenhaeuser/Publikationen/Downloads-Krankenhaeuser/tiefgegliederte-diagnosedaten5231301177015.xlsx?_blob=publicationFile; Zugriff am 14.07.2019.

Steiger A, Weber F, Benkert O: Medikamente zur Behandlung von Schlafstörungen. In: Benkert O, Hippius H (Hrsg.): Kompendium der Psychiatrischen Pharmakotherapie. Springer, Berlin 2019, 555-640

Stewart D, Mair A, Wilson M, Kardas P, Lewek P, Alonso A, McIntosh J, MacLure K, SYMPATHY consortium (2017): Guidance to manage inappropriate polypharmacy in older people: systematic review and future developments. Expert Opin Drug Saf 16, 203-213

Stockl KM, Le L, Zhang S, Harada AS (2010): Clinical and economic outcomes associated with potentially inappropriate prescribing in the elderly. Am J Manag Care 16, e1-10

Störmer M, Karaus M (2013): Qualitätsbericht 2013 - Evangelisches Krankenhaus Göttingen-Weende gGmbH. https://g-ba-qualitaetsberichte.de/api/file/download/260310447-00-2013xml.pdf; Zugriff am 26.04.2019. 
Tamburri LM, DiBrienza R, Zozula R, Redeker NS (2004): Nocturnal care interactions with patients in critical care units. Am J Crit Care 13, 102-113

Tamrat R, Huynh-Le MP, Goyal M (2014): Non-pharmacologic interventions to improve the sleep of hospitalized patients: a systematic review. J Gen Intern Med 29, 788-795

The American Geriatrics Society 2012 Beers Criteria Update Expert Panel (2012): American Geriatrics Society updated Beers Criteria for potentially inappropriate medication use in older adults. J Am Geriatr Soc 60, 616-631

The American Geriatrics Society 2015 Beers Criteria Update Expert Panel (2015): American Geriatrics Society 2015 updated Beers Criteria for potentially inappropriate medication use in older adults. J Am Geriatr Soc 63, 2227-2246

The American Geriatrics Society 2019 Beers Criteria Update Expert Panel (2019): American Geriatrics Society 2019 updated AGS Beers Criteria for potentially inappropriate medication use in older adults. J Am Geriatr Soc 67, 674-694

Tom SE, Wickwire EM, Park Y, Albrecht JS (2016): Nonbenzodiazepine sedative hypnotics and risk of fall-related injury. Sleep $\underline{39}, 1009-1014$

Trewin VF, Lawrence CJ, Veitch GBA (1992): An investigation of the association of benzodiazepines and other hypnotics with the incidence of falls in the elderly. J Clin Pharm Ther $\underline{17}, 129$ 133

Turnheim K (2003): When drug therapy gets old: pharmacokinetics and pharmacodynamics in the elderly. Exp Gerontol 38, 843-853

Vasudev A, Shariff SZ, Liu K, Burhan AM, Herrmann N, Leonard S, Mamdani M (2015): Trends in psychotropic dispensing among older adults with dementia living in long-term care facilities: 2004-2013. Am J Geriatr Psychiatry 23, 1259-1269

Wedmann F, Himmel W, Nau R (2019): Medication and medical diagnosis as risk factors for falls in older hospitalized patients. Eur J Clin Pharmacol 75, 1117-1124

Wesselius HM, Ende ES van den, Alsma J, Maaten JC ter, Schuit SCE, Stassen PM, Vries OJ de, Kaasjager KHAH, Haak HR, Doormaal FF van, et al. (2018): Quality and quantity of sleep and factors associated with sleep disturbance in hospitalized patients. JAMA Intern Med $\underline{178}$, $1201-1208$

Wilson MG, Lee TC, Hass A, Tannenbaum C, McDonald EG (2018): EMPOWERing hospitalized older adults to deprescribe sedative hypnotics: a pilot study. J Am Geriatr Soc 66, 1186-1189

Wilson S, Nutt D, Alford C, Argyropoulos S, Baldwin D, Bateson A, Britton T, Crowe C, Dijk D, Espie C, et al. (2010): British Association for Psychopharmacology consensus statement on evidence-based treatment of insomnia, parasomnias and circadian rhythm disorders. J Psychopharmacol (Oxf) 24, 1577-1601

Wirtz M (2020): Dorsch Lexikon der Psychologie - Quasi-Experiment. https://portal.hogrefe.com/dorsch/quasi-experiment; Zugriff am 27.12.2020.

Young JS, Bourgeois JA, Hilty DM, Hardin KA (2008): Sleep in hospitalized medical patients, Part 1: Factors affecting sleep. J Hosp Med $\underline{3}, 473-482$ 